ibw-Studie

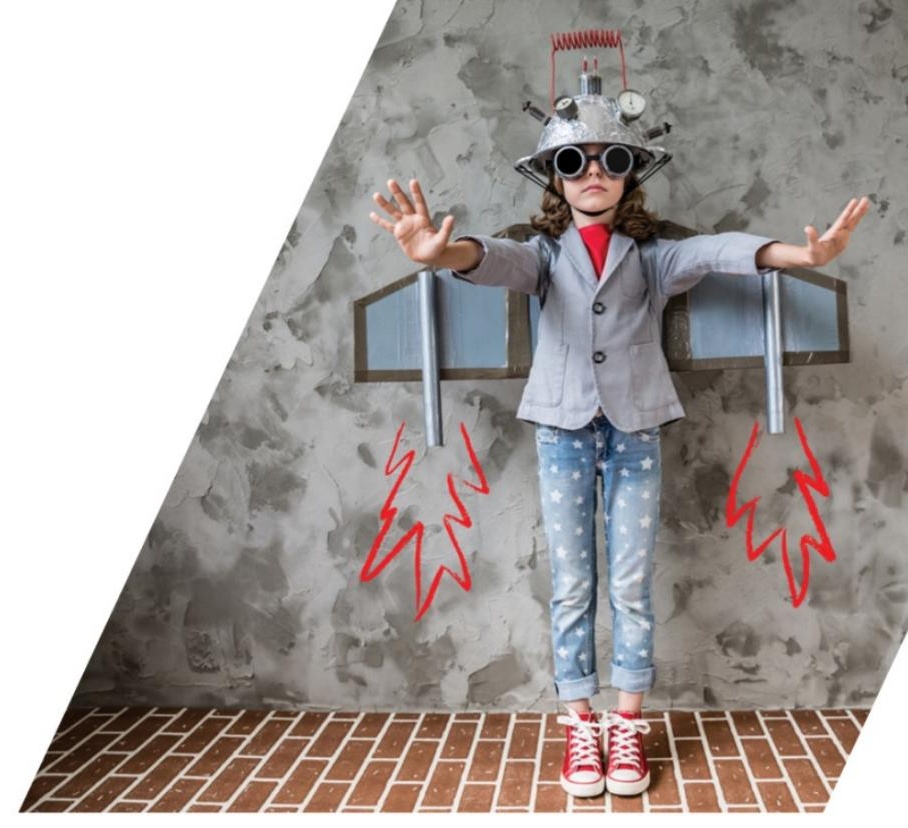

\title{
Review \\ Talente regional
}

Benjamin Gruber

Kurt Schmid 


\section{Impressum}

Review Talente regional

ibw-Studie, Wien 2018

Benjamin Gruber, Kurt Schmid

ISBN 978-3-903210-57-8

Zusätzlich zu dieser Studie wurden Kurzbeschreibungen der abgeschlossenen Projekte erstellt, die auf der open4innovation Plattform (www.open4innovation.at) abrufbar sind. Autorlnnen dieser Projektbeschreibungen sind Silvia Seyer-Weiß und Emanuel Van den Nest.

Medieninhaber und Herausgeber:

ibw

Institut für Bildungsforschung der Wirtschaft

ibw Austria - Research \& Development in VET

(Geschäftsführer: Mag. Thomas Mayr)

Rainergasse 38 | 1050 Wien

+43154516 71-0

www.ibw.at

ZVR-Nr.: 863473670

Foto (Titelseite)

Quelle: fotolia.com, Urheber: Sunny studio, Foto-ID: \#120704794

Kontakt

Benjamin Gruber, gruber@ibw.at

Diese Studie wurde im Auftrag des Bundesministeriums für Verkehr, Innovation und Technologie erstellt.

= Bundesministerium

Verkehr, Innovation

und Technologie 


\section{Inhalt}

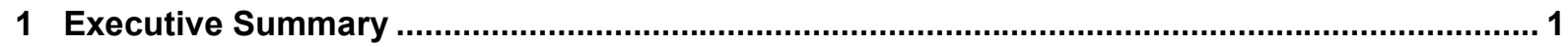

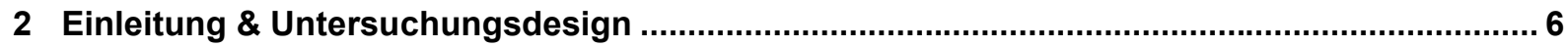

3 Deskriptive Projektübersicht anhand der Förderdaten ....................................................... 8

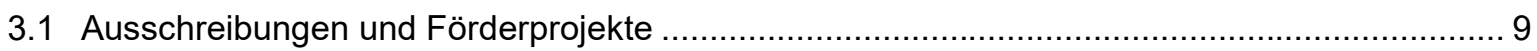

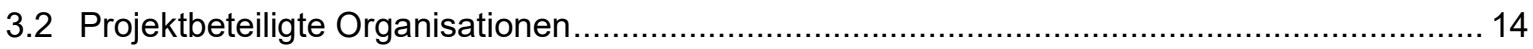

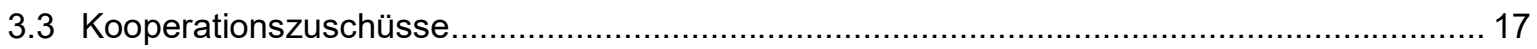

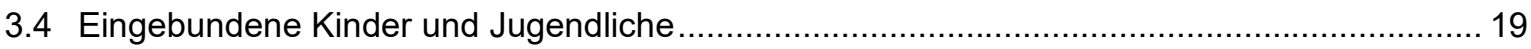

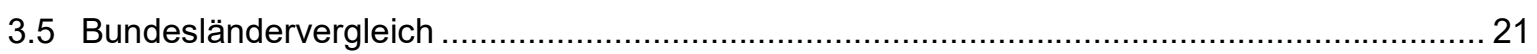

4 Quantitative Befragung der Projektbeteiligten...................................................................... 22

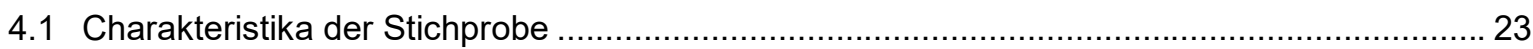

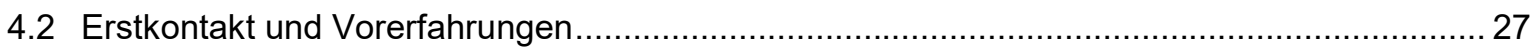

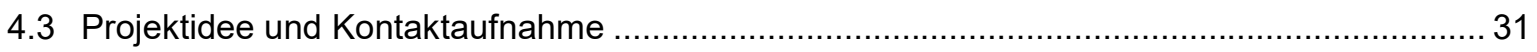

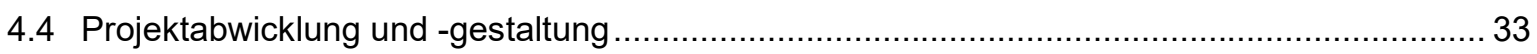

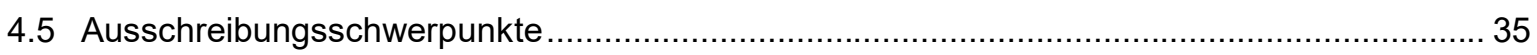

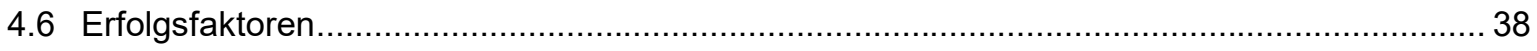

4.7 Wirkungen der Projektaktivitäten und Kooperationszuschüsse ........................................... 41

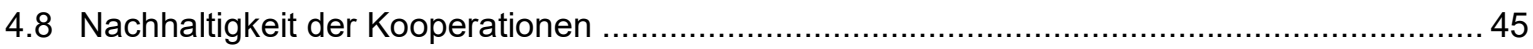

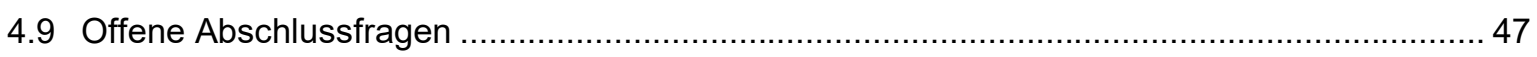

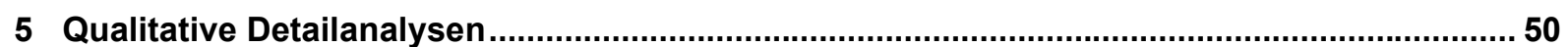

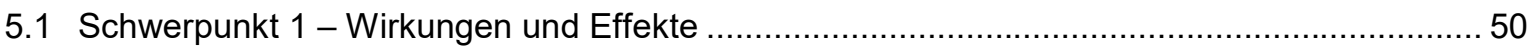

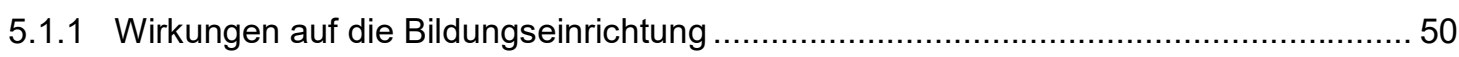

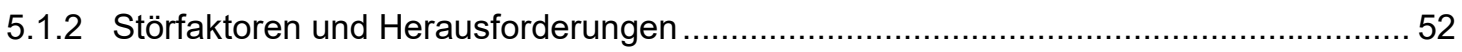

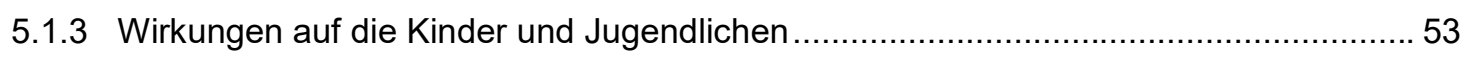

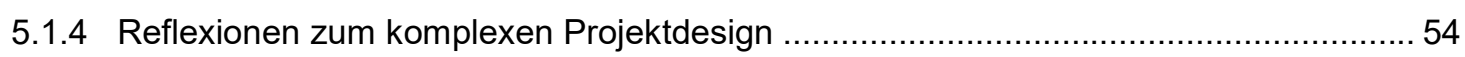

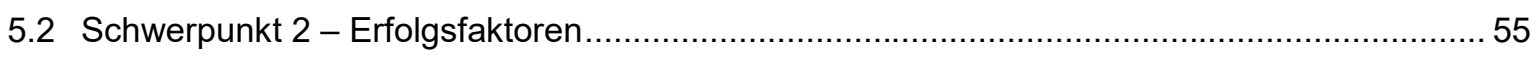

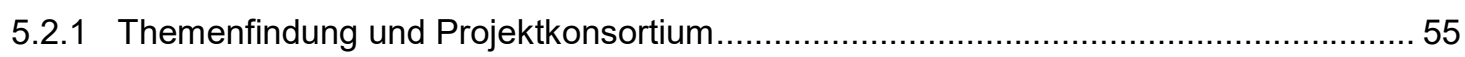

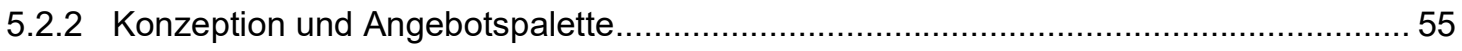

5.2.3 Einbindung und Unterstützung der Bildungseinrichtungen ........................................ 56

5.2.4 Unterschiede in Bezug auf Zusammensetzung und Ausrichtung .............................57

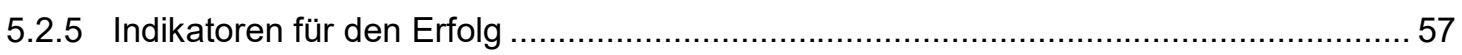

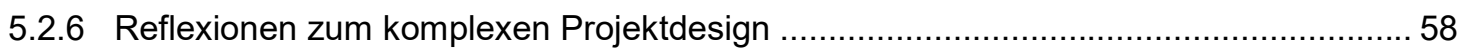

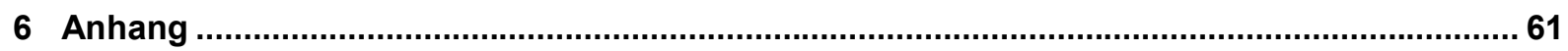

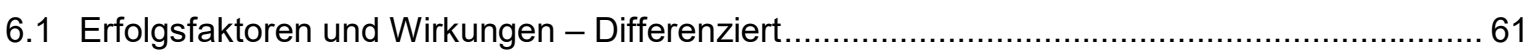

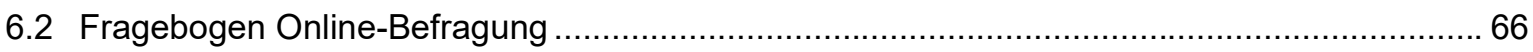

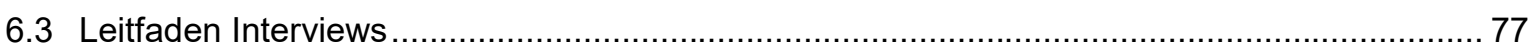





\section{Executive Summary}

„Talente regional fördert Projekte, die Kindern und Jugendlichen ermöglichen, sich über einen längeren Zeitraum mit Forschung, Technologie und Innovation (FTI) in den Bereichen Naturwissenschaft und Technik auseinanderzusetzen. Kinder und Jugendliche beschäftigen sich mit spannenden Themen, forschen, experimentieren und lernen Tätigkeiten und Berufsbilder in FTI kennen." (www.ffg.at/talente-regional)

Seit 2010 wurden im Rahmen von „Talente regional“ bzw. "generation innovation Regionen“ sieben Ausschreibungen mit insgesamt 135 Projekten durchgeführt. Dabei wird der Aufbau von regionalen Kooperationen zwischen (vor-)schulischen Bildungseinrichtungen und PartnerInnen aus Wirtschaft und Forschung gefördert, um Kinder und Jugendliche vom Kindergarten bis zur Matura bei der Auseinandersetzung mit Forschungs- und Innovationsthemen zu unterstützen.

Da ein Großteil der Projekte inzwischen abgeschlossen werden konnte, wurde vom Bundesministerium für Verkehr, Innovation und Technologie (BMVIT) ein Review-Prozess in Form dieser Studie beauftragt. Es wurde sowohl ein Überblick über die gesamte Projektlandschaft anhand wichtiger Eckdaten erstellt, als auch der Erfahrungsschatz der an den Projekten Beteiligten erfasst. Ziel ist es, die verschiedenen im Projekt gewonnen Erkenntnisse und Ergebnisse sowie deren langfristige Auswirkungen sichtbar zu machen. Neben einer deskriptiven Auswertung der Förderdaten wurden sowohl quantitative (Online-Befragung) als auch qualitative (leitfadengestützte Interviews) Methoden eingesetzt.

\section{Deskriptive Projektübersicht anhand der Förderdaten}

Auf Basis der Förderdaten wurde eine deskriptive Projektübersicht erstellt, mit der vor allem die Entwicklung der „Talente regional“-Ausschreibungen im Zeitverlauf dargestellt werden kann. So hat sich die Anzahl der jährlichen Projekteinreichungen zwischen 2010 und 2016 fast verdreifacht. Der Anteil der geförderten Projekte über alle Ausschreibungen liegt bei $41 \%$. Jährlich werden somit rund 16 bis 23 Projekte gefördert. Bis auf die Jahre 2010 (78\%) und 2014 (82\%) wurde das Budget der einzelnen Ausschreibungen fast gänzlich ${ }^{1}$ (mindestens 95\%) ausgeschöpft.

Den mit Abstand größten Anteil an den Beteiligten ${ }^{2}$ machen die (vor-)schulischen Bildungseinrichtungen mit 55\% aus. Mit über 30 Prozentpunkten Abstand folgen die Unternehmen mit 22\%, Forschungseinrichtungen mit $17 \%$ sowie Vereine, Länder und Gemeinden mit $7 \%$. Diese Verteilung bleibt aufgrund der vorgegebenen Anforderungen in den Ausschreibungen im Zeitverlauf nahezu unverändert.

Jede dritte Konsortialführung wurde von einem Unternehmen übernommen. Danach folgen hochschulische, Non-Profit- sowie außeruniversitäre Einrichtungen mit 16-18\%. Kompetenz-, Technologieund Impulszentren sind bei $13 \%$ der Projekte als Konsortialführung tätig.

Im Durchschnitt wurden (2010-2014) pro Projekt 388 Kinder und Jugendliche eingebunden. Die Geschlechterverteilung ist in nahezu allen Ausschreibungen (außer im Jahr 2010) gleichverteilt und schwankt im Zeitverlauf kaum.

\footnotetext{
${ }^{1}$ Aufgrund der fixen Projektkosten ist eine vollständige Ausschöpfung der Fördermittel nicht möglich.

2 Einreichberechtigt sind Hochschulen (Universitäten, Fachhochschulen und Pädagogische Hochschulen), außeruniversitäre Forschungseinrichtungen, Intermediäre (Einrichtungen des Technologietransfers: Gründer- und Technologiezentren, Unternehmenscluster, Science Center, Regionalmanagements) und Unternehmen. Zudem spielen (vor-)schulische Bildungseinrichtungen eine zentrale Rolle bei der Umsetzung der Projektaktivitäten.
} 
Grosso modo deckt sich die regionale Verteilung der Projekte (Basis Konsortialführung) mit der demografischen Struktur Österreichs. Bei der regionalen Gegenüberstellung der Verteilung der Projekte (Basis Konsortialführung), der Projektbeteiligten und der eingebundenen Kinder und Jugendlichen offenbart sich, dass in Wien tendenziell eher weniger Organisationen teilgenommen haben. In Oberösterreich, der Steiermark sowie Vorarlberg konnten verhältnismäßig viele Kinder und Jugendliche erreicht werden.

\section{Quantitative Befragung der Projektbeteiligten}

Die im Rahmen der Studie durchgeführte Online-Befragung ${ }^{3}$ ergab, dass die Projektidee bzw. der Impuls zur Beteiligung an der Ausschreibung zum Großteil (71\%) von den Konsortialführungen selbst kam. Die Kontaktaufnahme zu den KonsortialpartnerInnen (vor allem zu den wissenschaftichen Organisationen) zeigt ein eher ausgeglichenes Bild. Es wurde nahezu im selben Ausmaß sowohl auf neue Kontakte, als auch auf bestehende Netzwerke zurückgegriffen. Bei den Bildungseinrichtungen handelt es hingegen vermehrt um neue Kontakte, die im Rahmen des Projektes entstanden sind.

Der Grad der Zufriedenheit bezüglich der Ausschreibung sowie der konkreten Abwicklung ist enorm hoch und liegt deutlich über $80 \%$ („sehr zufrieden“ und „zufrieden“ kombiniert). Ähnliches gilt für die Bewertung des Projektkonsortiums, die fast ausschließlich positiv war. Und auch die grundsätzliche Eignung der Ziele und Anforderungen von "Talente regional“ wird durchwegs positiv beurteilt und liegt deutlich über $80 \%$ („trifft zu“ und „trifft eher zu“ kombiniert). Diese hohe Zufriedenheit ist besonders bemerkenswert, da die definierten Ziele und Anforderungen vom Großteil der Befragten als anspruchsvoll eingestuft werden (fast ein Fünftel der Befragten stuft sie als "sehr" und weitere zwei Drittel als „eher“ anspruchsvoll ein).

Bei der Bewertung der Erfolgsfaktoren werden eine Vielzahl an relevanten Kriterien für die gelungene Umsetzung der Projekte sowie Aktivitäten identifiziert. Dem eigenen Experimentieren sowie dem persönlichen Kontakt zu den ExpertInnen und der Besuch von wissenschaftlichen Organisationen wird eine besonders bedeutende Rolle beigemessen, um Kinder und Jugendliche zu begeistern. Wesentlich ist zudem die Vorbereitung auf die Aktivitäten im Unterricht (bspw. gestützt durch die Anwendung passender Unterrichtsmaterialien).

Kooperationszuschüsse setzen wichtige Impulse zur Verbreitung und Vernetzung der diversen PartnerInnen (Bildungseinrichtungen, Unternehmen, Forschungsorganisationen) mit dem Ziel, innovative Unterrichtsprojekte im Bereich Naturwissenschaft und Technik in den Kindergärten und Schulen durchzuführen. Insbesondere können dadurch wesentlich mehr Kinder und Jugendliche erreicht werden. Und dies mit einem vergleichsweise geringen formalen Aufwand.

Die Wirkungen der Aktivitäten wurden durchwegs sehr positiv bewertet. Bei Kindern und Jugendlichen zeigen sich positive Effekte in Bezug auf Interesse, Kenntnisse sowie einem realitätsnahen Einblick in die Tätigkeiten und Berufe in Naturwissenschaft und Technik. PädagogInnen lernen neue Methoden kennen, wie das Interesse der Kinder und Jugendlichen gesteigert werden kann und werden angeregt Projektarbeit in den Unterricht zu integrieren. Weiters haben sie die Möglichkeit einen realitätsnahen Einblick in die Unternehmen zu bekommen. Auf der Ebene der Bildungseinrichtungen kommt es, neben der Stärkung der schulinternen Zusammenarbeit und des Stellenwerts von Naturwissenschaft und Technik, zu Verbesserungen der technischen Infrastruktur sowie der regionalen Vernetzung mit Wirtschaft und Forschung.

\footnotetext{
3 Insgesamt konnten 181 RespondentInnen zur Auswertung herangezogen werden. Bezogen auf die Anzahl der erreichten bzw. vorhandenen Kontakte entspricht dies einer Rücklaufquote von $66 \%$ und einem Abdeckungsgrad von $59 \%$.
} 
Bezüglich der Nachhaltigkeit gaben fast drei Viertel der Befragten ${ }^{4}$ an, nach dem Projektende weiterhin mit den Projektbeteiligten kooperiert zu haben. Die Zusammenarbeit mit Bildungseinrichtungen wird als besonders intensiv eingeschätzt. Die Formen der Zusammenarbeit sind vielfältig, zumeist wird zwar direkt an die Arbeiten des abgeschlossenen Projektes angeknüpft aber auch das pädagogische bzw. didaktische Konzept wird weiterentwickelt.

Die größten Herausforderungen im Rahmen der Organisation waren die Koordination, Abstimmung, und Terminfindung mit den Projektbeteiligten sowie das Zeitmanagement speziell mit den Bildungseinrichtungen. Bei der Umsetzung wurde die kindgerechte und spannende Übersetzung naturwissenschaftlicher und technischer Inhalte, sowie die Berücksichtigung der Aufnahmefähigkeit, Interessenlage und des Wissensstands der Zielgruppe am häufigsten genannt. Konkrete Verbesserungsvorschläge bezogen sich vor allem auf die einfachere Kontaktaufnahme, sowie verbesserte Einbindung und Zusammenarbeit mit den Bildungseinrichtungen.

Bei den positiven Erfahrungen, die die Projekte mit sich brachten, wurden vor allem die Begeisterung und der Forschergeist der Kinder und Jugendlichen (strahlende Kinderaugen und die große Neugier) sowie die positiven Rückmeldungen der PädagogInnen und Eltern in den Vordergrund gestellt. Erwähnenswerte Unterschiede zwischen den Ausschreibungen, Projekten bzw. Projektkonsortien gab es nur wenige. Grundsätzlich wurden die Ausschreibungen bis auf einzelne Anpassungen im Zeitverlauf sehr ähnlich wahrgenommen. Die formelle Abwicklung hat sich im Laufe der Ausschreibungen vereinfacht und die zu berücksichtigenden Kriterien sind, durch die Hinzunahme der Migrations- und Genderthematik, gestiegen.

\section{Qualitative Detailanalysen}

Durch die qualitativen Detailanalysen wurden die deskriptiven und quantitativen Analysen und Auswertungen abschließend auch noch durch konkrete Beschreibungen und detaillierte Erfahrungsberichte ergänzt.

Die PädagogInnen betonten, dass der Zugang zu Kontakten bzw. Netzwerken für die Bildungseinrichtung einen besonders großen Effekt bzw. Mehrwert hat. Dieser erleichtert es nicht nur Exkursionen und Besichtigungen durchzuführen sondern stärkt auch die Sichtbarkeit und Attraktivität des eigenen Schulstandortes. Einerseits durch die Verbreiterung der schulinternen Angebotspalette und andererseits durch die teilweise damit einhergehende Professionalisierung des Lehrpersonals.

Negative Auswirkungen auf der Ebene der Bildungseinrichtungen wurden von den befragten PädagogInnen nicht genannt. Allerdings gibt es durchaus zentrale Faktoren, die berücksichtigt werden müssen, um eine friktionsfreie Umsetzung der Aktivitäten gewährleisten zu können. Dabei spielt sowohl die thematische Passung, als auch die Übertragbarkeit der Inhalte in den Unterricht eine zentrale Rolle. Aber auch die pädagogischen Kompetenzen der ExpertInnen sowie die Motivation und Einstellung der Personen, die an den Aktivitäten beteiligt sind, sollten nicht unterschätzt werden.

Als zentrale Wirkung der Aktivitäten wird, wenig überraschend, die Steigerung des Interesses der Kinder und Jugendlichen an naturwissenschaftlichen und technischen Themengebieten genannt. Die PädagogInnen stellen dabei die durchdachte Konzeption der Experimente und Übungen sowie die abwechslungsreiche Aufbereitung in den Vordergrund. Durch die Projekte wird ein besonderes Setting erzeugt, welches es ermöglicht, eine gewisse methodische Offenheit zu gewährleisten und somit die Bereitschaft, gängige Muster aufzubrechen und Neues auszuprobieren, fördert.

${ }^{4}$ Es wurden ausschließlich RespondentInnen berücksichtigt, deren Projekte zum Befragungszeitpunkt abgeschlossen waren. 
Nicht zu unterschätzen ist auch der positive Effekt, den die Aktivitäten auf die Entwicklung des Selbstbildes bei den Kindern und Jugendlichen haben. Gerade durch die externe Aufmerksamkeit und Bestätigung bekommen die Leistungen eine gewisse Wertigkeit und die Teilnehmerlnnen werden ermutigt ihr Potenzial zu entfalten. Besonders förderlich sind dabei auch die verschiedenen Peer-Mentoring/Tutoring-Konzepte, die es vor allem den älteren Schülerlnnen erlauben in die Perspektive des Lehrenden einzutauchen.

Die PädagogInnen sind der Meinung, dass die verschiedenen Zielsetzungen im Rahmen der Projektkonzeption zumeist gut vereinbar und umsetzbar waren. Lediglich bei den Zielen der Geschlechterausgewogenheit sowie der Integration von Kindern und Jugendlichen unabhängig ihrer sozialen oder geographischen Herkunft fehlt es zum Teil an projektspezifischen Anknüpfungspunkten, die die Auswahl der passenden Methoden sowie des gewünschten Kontextes erleichtern würden.

Aber auch in der konkreten Umsetzung ergeben sich aufgrund des lokalen Umfelds verschiedene Beschränkungen. So ist es nicht in jeder Region möglich die passenden Role-Models zu finden oder die gleichmäßige Verteilung der Geschlechter bei den ExpertInnen zu erreichen.

Der Kooperationszuschuss wird sehr positiv beurteilt. Die Inanspruchnahme ist relativ unkompliziert, wodurch vor allem innovative Ideen und Initiativen, die oftmals an der Finanzierung und dem damit einhergehenden Aufwand scheitern, gefördert werden. Im Vergleich zu anderen Förderungen wird der Multiplikatoreffekt der eingesetzten Mittel als extrem hoch eingeschätzt.

Von den Befragten mit mehreren Projektteilnahmen werden vor allem die Wichtigkeit der Themenwahl und die Übertragbarkeit der Inhalte in den schulischen Kontext betont. Dazu kommen bereits bestehende Kooperationen und die pädagogischen Kompetenzen der Projektbeteiligten. Aber auch die interdisziplinäre Umsetzung und die Möglichkeit eine breite Palette an modularen Angeboten bzw. Formaten zur Verfügung zu stellen, werden als vorteilhafte Faktoren genannt.

Weiters wird die zentrale Rolle der Bildungseinrichtungen für den Erfolg der Aktivitäten hervorgehoben. In diesem Kontext spielen die möglichst frühe Einbindung der PädagogInnen in die Konzeption der Projektaktivitäten und projektspezifische Fortbildungen, die den Fokus auf naturwissenschaftliche und technische Themen sowie deren konkrete Vermittlung im Unterricht legen, eine besonders wichtige Rolle.

Als Erfolgsindikatoren werden von den Befragten vor allem qualitative Kriterien, die im Rahmen von persönlichen Rückmeldungen kommuniziert werden, genannt. Besonders der Stolz der Kinder und Jugendlichen auf die eigene Leistung und das dadurch gestärkte Selbstbewusstsein werden betont. Hinzu kommt die anhaltende Nachfrage der PädagogInnen nach Fortbildungen, die Vielzahl an intensiven Kooperationen sowie die Gründung von Organisationen (Vereine) im Rahmen der Projekte, deren Wirkungsbereich weit über die ursprüngliche Projektidee hinausgeht.

Die verschiedenen Zielsetzungen im Rahmen der Projekte waren laut den Befragten grundsätzlich gut vereinbar. Allerdings wurde die Berücksichtigung der Schwerpunkte Geschlechterausgewogenheit und der Integration von Kindern und Jugendlichen unabhängig ihrer sozialen oder geographischen Herkunft bei der Umsetzung der Projektaktivitäten als besonders herausfordernd und teilweise sogar als störend wahrgenommen.

Mit den Kooperationszuschüssen kann man die Bildungseinrichtungen in der Umgebung sehr gut ansprechen und verbreitert dadurch die regionale Bekanntheit der Projekte. Die finanzielle Unterstützung ermöglicht es den PädagogInnen die Infrastruktur (Geräte und Materialien) an den Bildungseinrichtungen auszubauen und fördert das Interesse an Exkursionen oder Besichtigungen teilzunehmen. 


\section{Kurzfazit}

Sowohl der hohe Ausschöpfungsgrad, als auch die große Zufriedenheit der Projektbeteiligten hinsichtlich Ausschreibungskriterien, Projektabwicklung sowie mit den Projektkonsortien, belegen die hohe Attraktivität von „Talente regional“.

Die Rückmeldungen der Befragten zeichnen ein sehr positives Bild der erzielten Effekte, nicht nur auf die primäre Zielgruppe der Kinder und Jugendlichen, sondern auch auf die PädagogInnen sowie auf die Schulentwicklung.

„Talente regional“ unterstützt die österreichweite Entwicklung regionaler Initiativen und Netzwerke und liefert somit einen wichtigen Beitrag zur Stärkung des Interesses an und der Kenntnisse über Naturwissenschaft und Technik. 


\section{Einleitung \& Untersuchungsdesign}

Das Entdecken und Fördern von (jungen) Talenten, vor allem im naturwissenschaftlichen und technischen Bereich ist eine Grundvoraussetzung, um den Anforderungen, die eine moderne Informationsgesellschaft mit sich bringt, in Zukunft gerecht zu werden. Der Förderschwerpunkt „Talente" des Bundesministeriums für Verkehr, Innovation und Technologie (BMVIT) setzt genau an dieser Prämisse an und versucht die Rahmenbedingungen für Forscherlnnen in Österreich zu verbessern und somit die Entwicklung von Talenten voranzutreiben.

Der Förderschwerpunkt „Talente“ ist in die folgenden drei Bereiche gegliedert:

\section{Talente entdecken: Nachwuchs}

- Praktika für Schülerinnen und Schüler - Vier Wochen Naturwissenschaft und Technik

- Talente regional - Kinder, Unternehmen und die Welt der Forschung

\section{Talente nützen: Chancengleichheit}

- FEMtech Karriere-Check für KMU - Genderanalyse

- FEMtech Karriere - Chancengleichheit in der angewandten Forschung

- FEMtech Praktika für Studentinnen - Einstieg in die Forschungskarriere

- FEMtech Forschungsprojekte - Gendergerechte Innovation

\section{Talente finden: Forscherinnen und Forscher}

- Die österreichische Jobbörse für Forschung, Entwicklung und Innovation

- Karriere-Grants für Vorstellungsgespräche, Umzug nach Österreich und Integration des Partners/der Partnerin

Ein integraler Bestandteil des Förderschwerpunkts ist „Talente regional - Kinder, Unternehmen und die Welt der Forschung“. Es fördert den Aufbau von regionalen Kooperationen zwischen (vor)schulischen Bildungseinrichtungen und PartnerInnen aus Wirtschaft und Forschung, damit diese Kinder und Jugendliche, vom Kindergarten bis zur Matura, bei der Auseinandersetzung mit Forschungs- und Innovationsthemen unterstützen. Dazu werden Maßnahmen und Aktivitäten durchgeführt, die den TeilnehmerInnen über einen längeren Zeitraum den Zugang zu praktischen Erfahrungen im wissenschaftlichen und betrieblichen Umfeld sowie zu Interaktionsmöglichkeiten mit ExpertInnen ermöglichen. Die Kinder und Jugendlichen beschäftigen sich mit spannenden Themen, forschen, experimentieren und lernen Tätigkeiten und Berufsbilder im Bereich Forschung, Technologie und Innovation kennen. ${ }^{5}$ Die Abwicklung von „Talente regional“ erfolgt von der Österreichischen Forschungsförderungsgesellschaft mbH (FFG).

Einreichberechtigt sind (als Konsortialführung bzw. Konsortialpartnerln) Organisationen, die einen Standort in Österreich haben. Dazu zählen Hochschulen (Universitäten, Fachhochschulen und Pädagogische Hochschulen), außeruniversitäre Forschungseinrichtungen, Intermediäre (Einrichtungen des Technologietransfers: Gründer- und Technologiezentren, Unternehmenscluster, Science Center, Regionalmanagements) und Unternehmen. Das Projektkonsortium besteht aus der Konsortialführung, die sich um das Projektmanagement sowie die Kommunikation mit der FFG und den ProjektpartnerInnen kümmert sowie aus wissenschaftlichen PartnerInnen und UnternehmenspartnerInnen, die das notwendige Know-how aus Forschung und Wirtschaft einbringen. Die (vor-) schulischen Bildungseinrichtungen als Projektbeteiligte spielen zwar eine zentrale Rolle bei der Umsetzung der Projektaktivitäten, sind aber nicht Teil des Konsortiums.

${ }^{5} \mathrm{vgl}$. www.ffg.at/sites/defaultffiles/downloads/call/talente regional 6as ausschreibungsleitfaden 1.pdf 
Zusätzlich standen jedem Projekt (seit 2011) maximal 10 Kooperationszuschüsse zur Verfügung, die dazu dienten, die Bekanntheit von „Talente regional“ zu verbessern und innovative Unterrichtsprojekte an Kindergärten und Schulen zu fördern. Es handelt sich dabei um Pauschalförderungen (je 1.000 Euro) für weitere Bildungseinrichtungen, die nicht direkt am Projekt beteiligt sind.

Im Rahmen von "Talente regional" bzw. "generation innovation Regionen"6 wurden seit dem Jahr 2010 in sieben Ausschreibungen insgesamt 135 Projekte vom Bundesministerium für Verkehr, Innovation und Technologie (BMVIT) gefördert. Da ein Großteil der Projekte (Ausschreibungen aus den Jahren 2010 bis 2014) inzwischen abgeschlossen werden konnte, wurde ein Review-Prozess in Form einer umfassenden Studie vom BMVIT beauftragt.

Im Rahmen der vorliegenden Studie soll sowohl ein Überblick über die gesamte Projektlandschaft anhand wichtiger Eckdaten erstellt, als auch der Erfahrungsschatz der an den Projekten Beteiligte dargestellt werden. Ziel ist es, die verschiedenen im Projekt gewonnenen Erkenntnisse, Ergebnisse sowie deren langfristige Auswirkungen zu erfassen und sichtbar zu machen. Dabei kamen neben der deskriptiven Auswertung der Förderdaten sowohl quantitative (Online-Befragung) als auch qualitative (leitfadengestützte Interviews) Methoden zum Einsatz. ${ }^{7}$

In einem ersten Schritt wurden die wichtigsten Charakteristika im Rahmen einer deskriptiven Projektübersicht (vgl. Abschnitt 3) analysiert. Als Datenbasis wurden die Projektinformationen (Förderdaten der österreichischen Forschungsförderungsgesellschaft (FFG)) sowie die Projektberichte (Zwischen- und Endbericht) herangezogen. Die Ergebnisse dieses ersten Analyseschritts wurden im Rahmen eines Feedback-Meetings (mit VertreterInnen des BMVIT, der FFG und des Bewertungsgremium) präsentiert und diskutiert. Dabei wurden auch die thematischen Schwerpunktsetzungen für die darauf folgende quantitative Erhebungsphase festgelegt.

In einer quantitativen Befragung (Online-Befragung) wurden Projektbeteiligte zu ihren Projekterfahrungen befragt, um Gemeinsamkeiten, aber auch allfällige Unterschiede auf einer repräsentativen Basis erfassen zu können (Abschnitt 4). Zentrale Themenfelder waren neben der Zufriedenheit mit der Projektabwicklung und -gestaltung vor allem die Vereinbarkeit der Ausschreibungsschwerpunkte, Erfolgsfaktoren und Wirkungen der Projektaktivitäten und Kooperationszuschüsse sowie die Nachhaltigkeit der Kooperationen. Die Ergebnisse dieser Erhebungsphase wurden im Rahmen eines Meetings (mit VertreterInnen des BMVIT, der FFG und des Bewertungsgremiums) präsentiert und diskutiert, um die thematischen Schwerpunkte der zweiten Erhebungsphase festzulegen.

In einer abschließenden Erhebungsphase wurden qualitative Detailanalysen (Abschnitt 5) auf Basis von leitfadengestützten Interviews durchgeführt. Ausgehend von den Ergebnissen der quantitativen Erhebung wurden dabei jene thematischen Schwerpunkte, die für die Weiterentwicklung bzw. Bewertung von "Talente regional" hohe Relevanz haben, vertieft. Zentrale Themenfelder waren neben den (Aus-)Wirkungen der Projektaktivitäten die Erfolgsfaktoren sowie Indikatoren für den Erfolg der Projekte, Reflexionen zum komplexen Projektdesign bzw. Irritationen und Störfaktoren. Als Zielgruppe wurden sowohl Vertreterlnnen von Bildungseinrichtungen als auch Projektbeteiligte, die an mehreren Talente regional-Projekten beteiligt waren, ausgewählt.

\footnotetext{
${ }^{6}$ Bezeichnung der Ausschreibung aus dem Jahr 2010. Alle nachfolgenden Ausschreibungen firmieren bereits unter dem Titel „Talente regional“.

7 Die Analysen der Studie konzentrieren sich somit ausschließlich auf geförderte Projekte. Einreichungen, bei denen es zu keiner Förderung gekommen ist, wurden nicht behandelt.
} 


\section{Deskriptive Projektübersicht anhand der Förderdaten}

Um einen Überblick über alle genehmigten Projekte zu bekommen, werden im Rahmen einer deskriptiven Übersicht relevante Charakteristika zu den Ausschreibungen, Projekten, Projektbeteiligten, Kooperationszuschüssen und erreichten Personen dargestellt. Als Datenbasis wurden die Projektdatenbank der FFG sowie die Projektanträge und -berichte (Zwischen- und Endbericht) herangezogen.

Der Überblick ermöglicht eine aggregierte und quantitative Darstellung (Mengengerüst) der bisherigen Ausschreibungen. Dabei werden verschiedenste Indikatoren wie bspw. die Anzahl der Einreichungen und geförderten Projekte, die durchschnittlich genehmigte Förderhöhe pro Projekt, aber auch die Verteilung auf die Bundesländer, Organisationsformen und Themenfelder dargestellt. Außerdem werden die Daten zu den Kooperationszuschüssen sowie den eingebundenen Kindern und Jugendlichen analysiert. Abschließend findet ein Bundeslandvergleich statt, indem die relative Verteilung der Projekte, Projektbeteiligten und eingebundenen Zielgruppen gegenübergestellt werden.

Die folgende Abbildung gibt einen ersten kurzen Überblick zu den Eckdaten von „Talente regional“. In insgesamt sieben Ausschreibungen gab es 327 Projekteinreichungen, wovon 135 Projekte als förderungswürdig eingestuft wurden. Im Rahmen dieser 135 geförderten Projekte wurden 766 Kooperationszuschüsse $^{8}$ vergeben und es haben 1.626 Projektbeteiligte $^{9}$ an den Projekten teilgenommen. Laut den Angaben, die die Konsortialführungen in den Endberichten gemacht haben, wurden 34.525 Kinder und Jugendliche ${ }^{10}$ in die verschiedenen Maßnahmen und Aktivitäten der Projekte eingebunden.

\section{Abbildung 1 Eckdaten zu „Talente regional“}

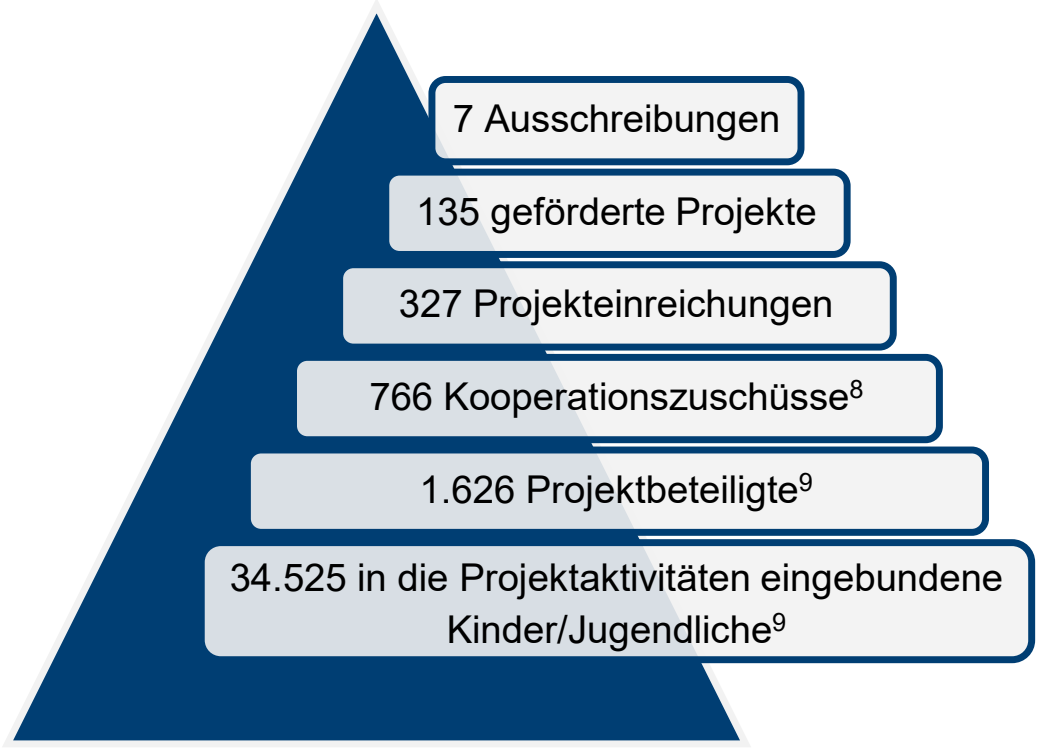

\footnotetext{
8 Die Kooperationszuschüsse wurden erstmalig im Jahr 2011 eingeführt und dann in allen Ausschreibungen beibehalten. Da zum Zeitpunkt der Auswertungen noch keine Endberichte aus dem Jahr 2015 und keine Zwischenberichte aus dem Jahr 2016 zur Verfügung standen, ist die tatsächliche Anzahl höher.

${ }^{9}$ Es handelt sich dabei um das Projektkonsortium (Konsortialführung, wissenschaftliche PartnerInnen und UnternehmenspartnerInnen) und Bildungseinrichtungen, die in Form einer Projektbeteiligung vertreten waren. Nicht berücksichtigt wurden Bildungseinrichtungen, die einen Kooperationszuschuss erhalten haben. Organisationen, die sich an mehreren Projekten beteiligt haben, werden mehrfach gezählt (ungefähr 200 Fälle von 2010-2016).

${ }^{10}$ Zum Zeitpunkt der Auswertungen standen noch keine Daten zu den eingebundenen Kindern und Jugendlichen aus den Jahren 2015 und 2016 zur Verfügung. Diese Anzahl bezieht sich somit auf die Ausschreibungen von 2010 bis 2014. Daten aus den Aktivitäten der Kooperationszuschüsse wurden nicht berücksichtigt.
} 


\subsection{Ausschreibungen und Förderprojekte}

Der folgende Abschnitt vergleicht nun die einzelnen Ausschreibungen in Bezug auf die Anzahl der Einreichungen und geförderten Projekte, den Anteil der geförderten Projekte an den Einreichungen, den Anteil der genehmigten Förderungen an den zur Verfügung stehenden Fördermitteln und die durchschnittlich genehmigte Förderhöhe pro Projekt. Weiters werden die einzelnen Charakteristika wie die Verteilung der Bundesländer, Organisationsformen und Themenfelder der Projekte dargestellt.

Betrachtet man die Anzahl der Einreichungen von 2010 und 2016, so fällt auf, dass sie sich im Zeitverlauf fast verdreifacht hat. Die Anzahl der geförderten Projekte hat sich hingegen wesentlich stabiler entwickelt, da dieser Wert von den zur Verfügung stehenden Fördermitteln abhängig ist.

\section{Grafik 1: Beteiligung an den Ausschreibungen}

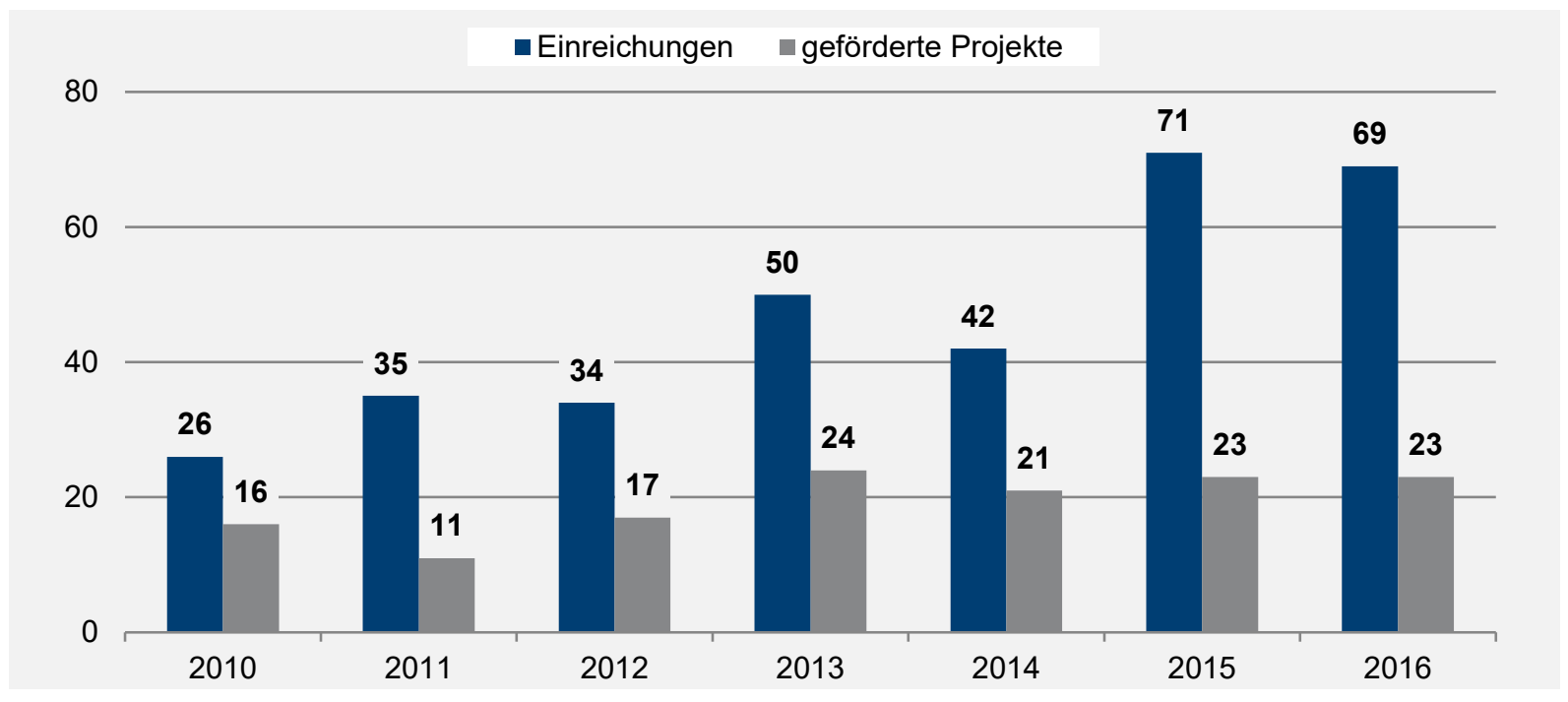

Quelle: Projektdatenbank der FFG (2010-2016)

Anhand des relativen Anteils der geförderten Projekte an den Einreichungen zeigt sich, dass es in Ausschreibungen aus den Jahren 2011, 2015 und 2016 ungefähr drei Mal so viele Einreichungen wie geförderte Projekte gegeben hat. Von 2012 bis 2014 liegt der Anteil mit 50\% etwas niedriger. Den größten Anteil an geförderten Projekten im Vergleich zu den Einreichungen gab es im Jahr 2010 mit über 60\%. Der Durchschnitt über alle Ausschreibungen zwischen 2010 und 2016 liegt bei $41 \%$.

\section{Grafik 2: Anteil der geförderten Projekte an den Einreichungen}

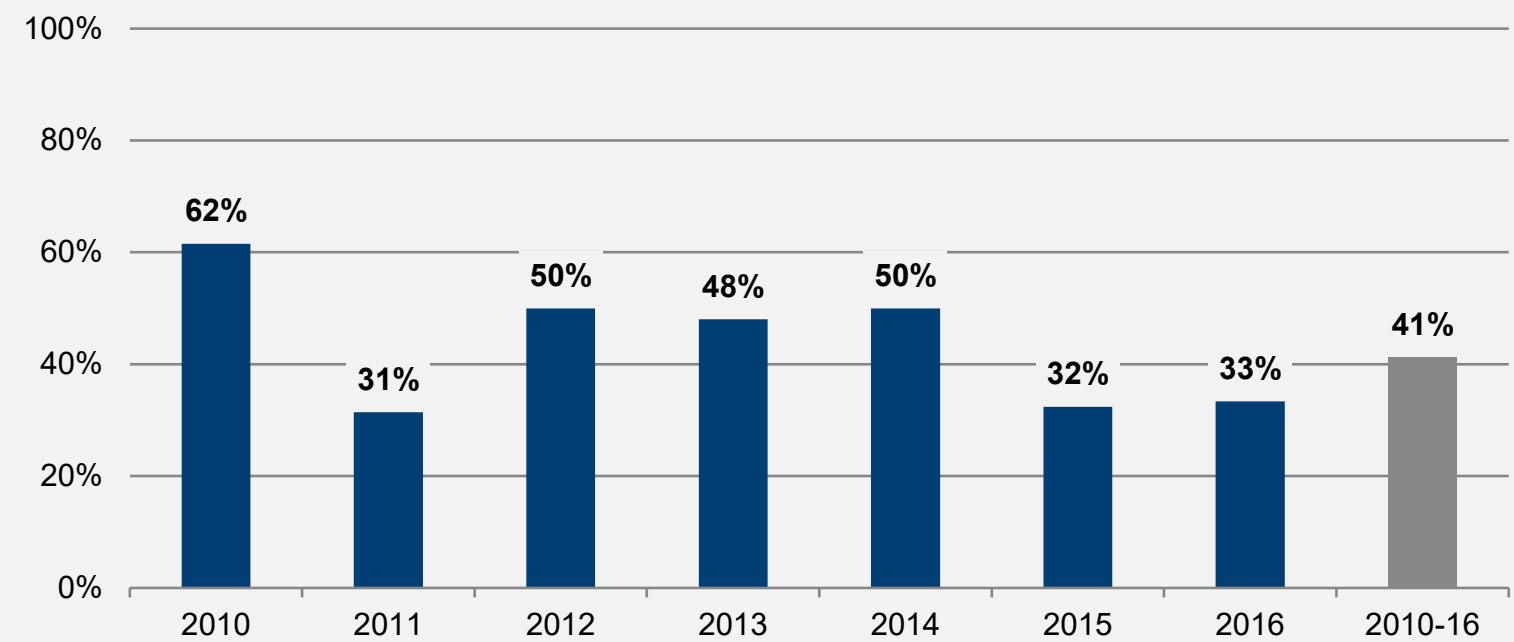

Quelle: Projektdatenbank der FFG (2010-2016) 
Wie man in der Grafik 3 erkennen kann, wurden die zur Verfügung stehenden Fördermittel beim Großteil der Ausschreibungen fast vollständig ausgeschöpft ${ }^{11}$. Lediglich in den Jahren 2010 und 2014 wären noch Kapazitäten für weitere Projekte vorhanden gewesen, da noch 22\% bzw. 18\% der für die jeweilige Ausschreibung zur Verfügung stehenden Fördermittel verfügbar waren. Der Durchschnitt über alle Ausschreibungen zwischen 2010 und 2016 liegt bei etwa 94\%.

\section{Grafik 3: Ausschöpfungsgrad der Ausschreibungen}

Anteil der genehmigten Förderungen an den zur Verfügung stehenden Fördermitteln

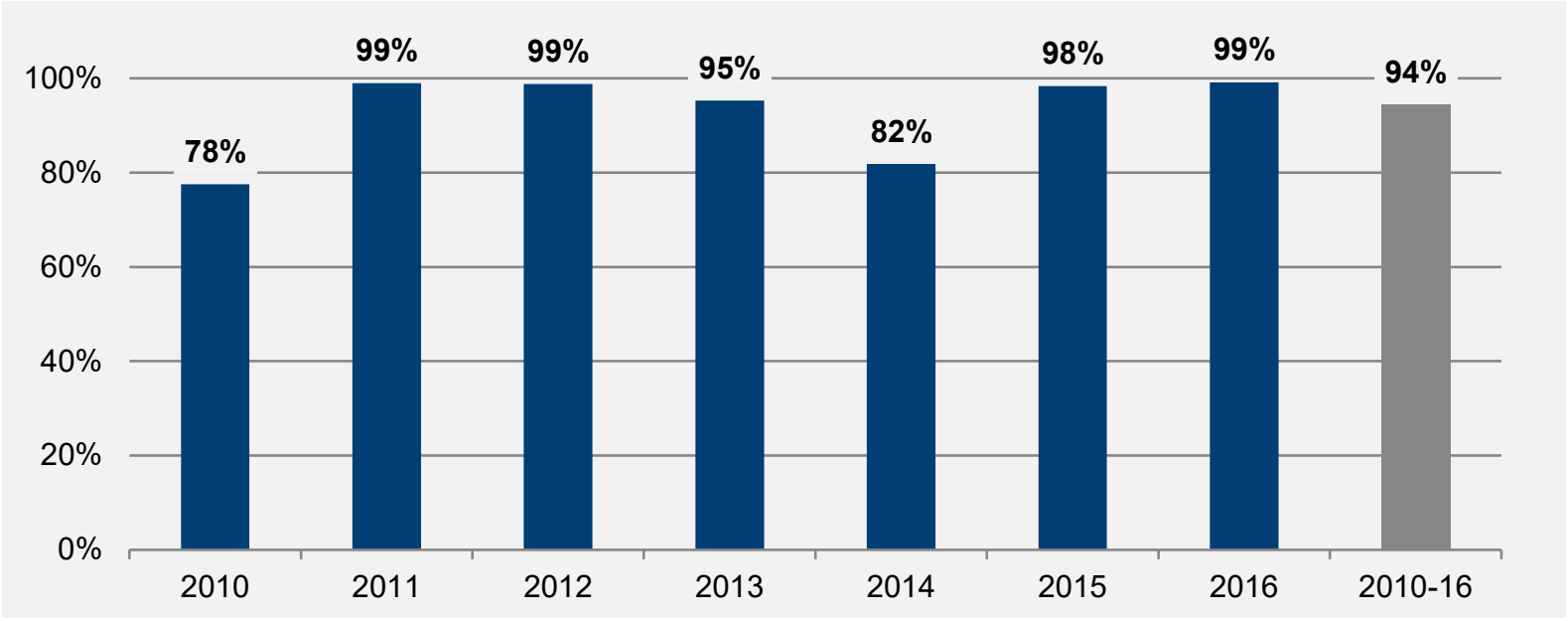

Quelle: Projektdatenbank der FFG (2010-2016)

Grafik 4 zeigt, wie sich die durchschnittlich genehmigte Förderhöhe im Zeitverlauf entwickelt hat, und dass die Projekte die maximale Förderhöhe je Projekt (dargestellt durch den grauen Strich über den blauen Balken) zumeist gänzlich ausgeschöpft haben.

Im Jahr 2010 lag die maximale Förderhöhe noch bei $45.000 €$. Zwischen 2011 und 2014 bei $60.000 €$ und 2015 und 2016 bei $130.000 €$. Mit der Erhöhung der Förderhöhe von $60.000 €$ auf $130.000 €$ ging auch eine Verdoppelung der Projektlaufzeit (von mindestens 12 auf 24 Monate und maximal 18 auf 36 Monate) einher.

\section{Grafik 4: Durchschnittlich genehmigte Förderhöhe pro Projekt}

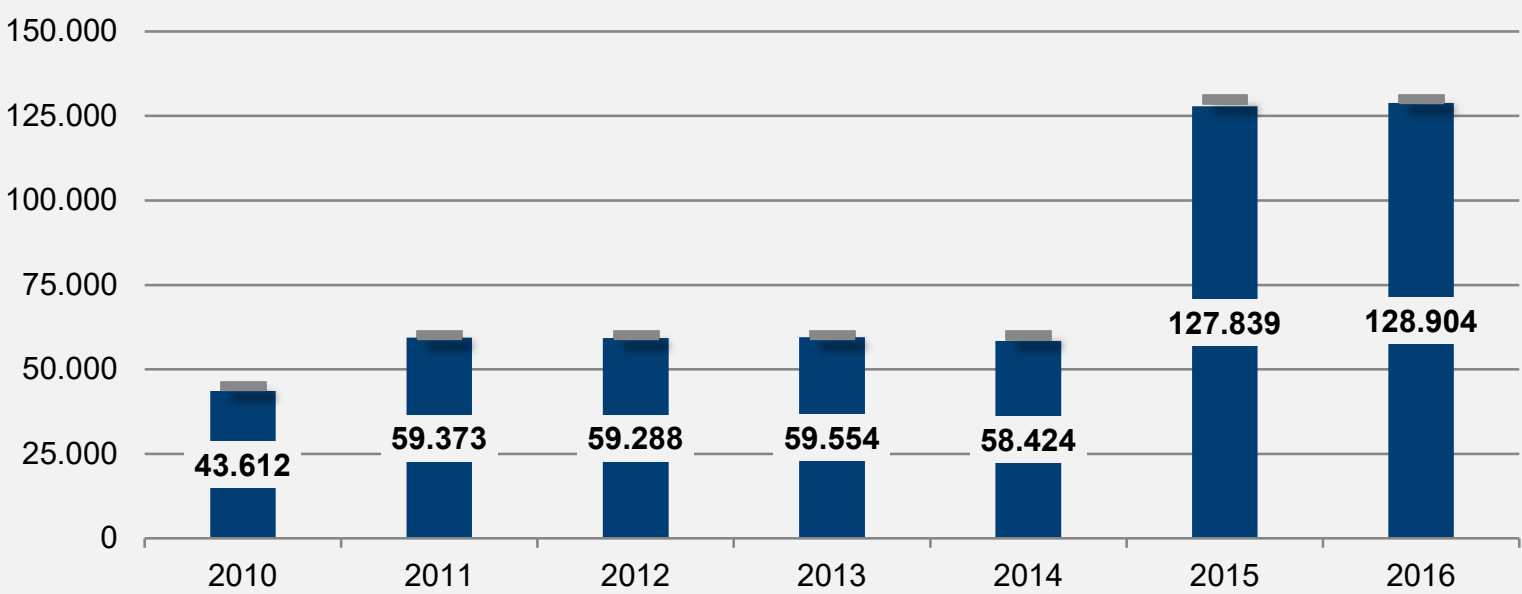

Quelle: Projektdatenbank der FFG (2010-2016)

${ }^{11}$ Aufgrund der fixen Projektkosten ist eine vollständige Ausschöpfung der Fördermittel nicht möglich. 
In jeweils einem Viertel der insgesamt 135 geförderten Projekte kommt die Konsortialführung entweder aus der Steiermark bzw. aus Wien. Danach folgen Oberösterreich, Niederösterreich und Tirol mit einem Anteil von 16\%, 13\% sowie 10\%. Im Burgenland bzw. in Kärnten liegt der Anteil bei 3\% bzw. $4 \%$. Der geringste Anteil, mit jeweils nur einem Prozent, entfältt auf Vorarlberg und Salzburg.

Stellt man diese Werte in Relation zum Bevölkerungsanteil der einzelnen Bundesländer, so offenbaren sich zum Teil relativ große Differenzen. So ist die Steiermark mit 12 Prozentpunkten besonders stark überrepräsentiert. Danach folgen Wien und Tirol mit 5 bzw. 2 Prozentpunkten. Bei den unterrepräsentierten Bundesländern sind die Differenzen eher geringer. So liegen die Werte in Kärnten, Vorarlberg, Salzburg und Niederösterreich zwischen 2 und 6 Prozentpunkten. Im Burgenland sind die Differenzen zwischen dem Anteil an den Projekten und dem Bevölkerungsanteil nur sehr gering ausgeprägt.

Grafik 5: Verteilung der Projekte auf die Bundesländer (Basis Konsortialführung) und Bevölkerungsanteile (Basis Gesamtbevölkerung)

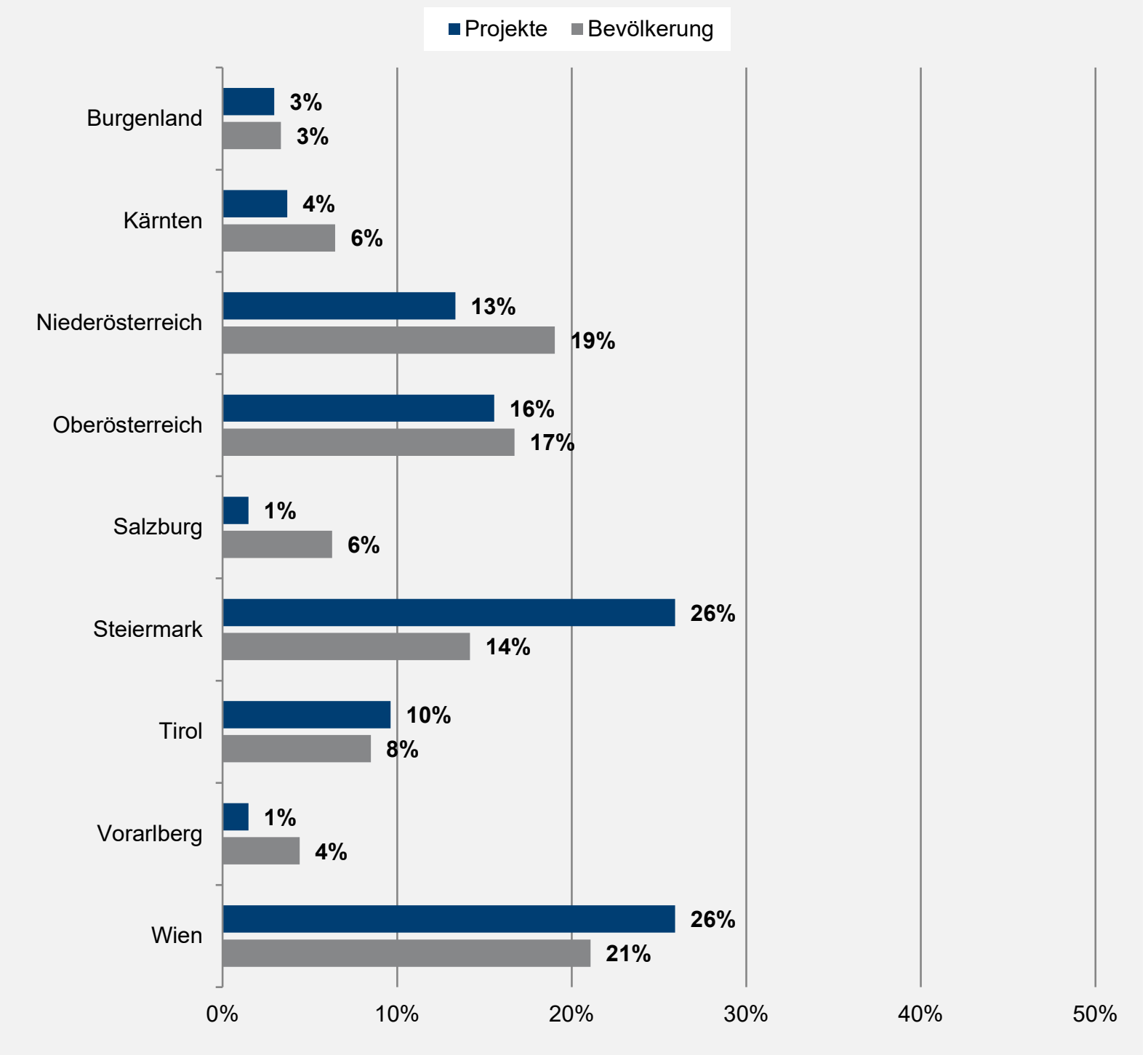

Quelle: Projektanträge und -berichte (2010-2016), n=135 ; Statistik Austria, Abgestimmte Erwerbsstatistik 2015

Interessant ist auch, wie sich die Bundesländer im Zeitverlauf verteilen, sprich ob es große Unterschiede zwischen einzelnen Ausschreibungen gab. Grundsätzlich lassen sich durchaus gewisse Schwankungen beobachten. So war die Steiermark in jedem Jahr, bis auf 2012, relativ stark vertreten. In Niederösterreich hingegen war man, bis auf 2010, verhältnismäßig schwach vertreten. 
Grafik 6: Verteilung der Projekte auf die Bundesländer (Basis Konsortialführung) differenziert nach Ausschreibung

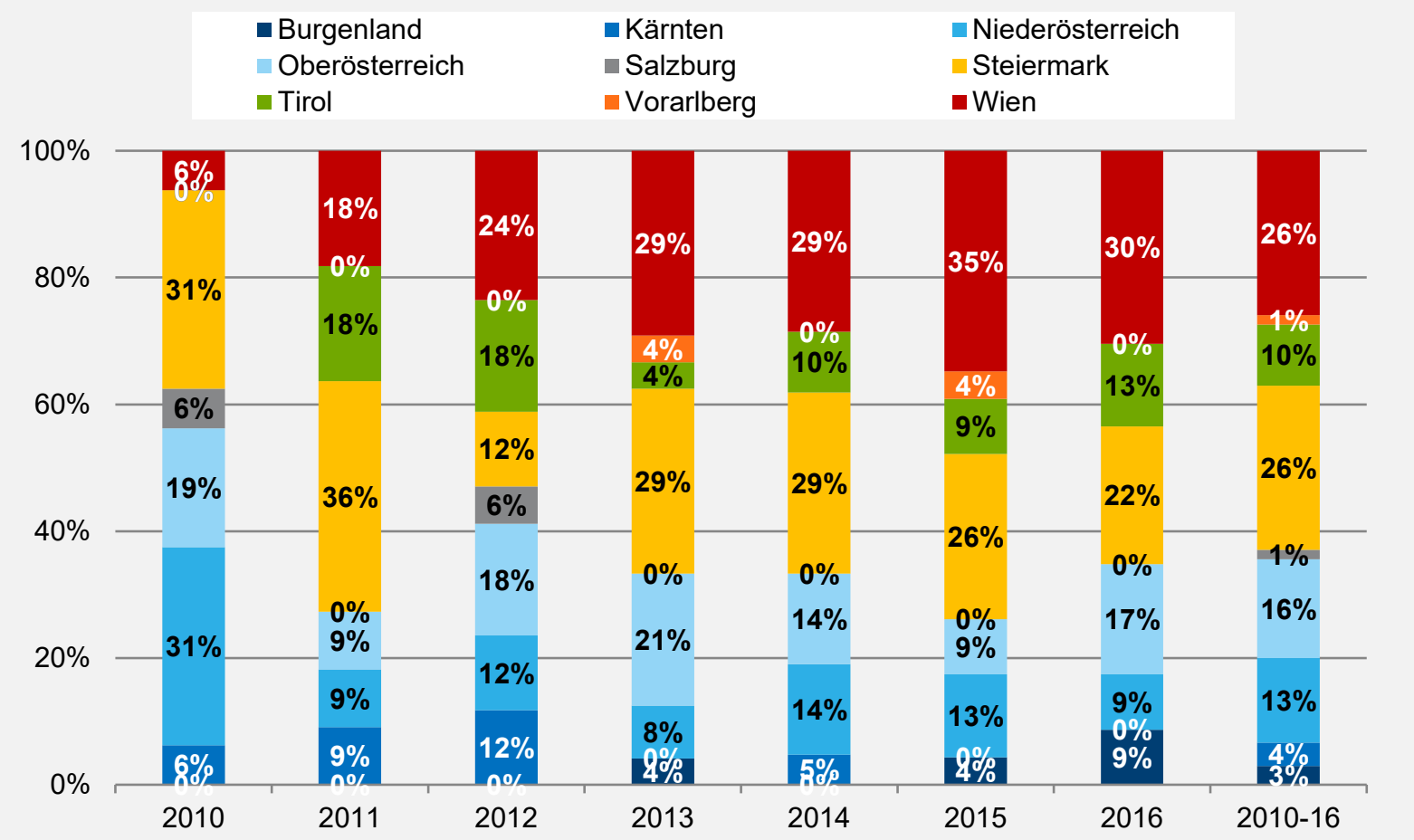

Quelle: Projektanträge und -berichte (2010-2016), n=135

Da in den Projektberichten aus dem Jahr 2010 keine systematisierten Informationen zu den Organisationsformen der Konsortialführung und den Themenfeldern der Projekte vorhanden sind, beziehen sich Grafik 7 und Grafik 8 auf Projekte ab der Ausschreibung aus dem Jahr 2011.

Betrachtet man die Organisationsformen der Konsortialführung aus den Projekten der Ausschreibungen 2011 bis 2016, so zeigt sich, dass Unternehmen mit ungefähr einem Drittel den mit Abstand größten Anteil ausmachen. Danach folgen hochschulische, Non-Profit- sowie außeruniversitäre Einrichtungen mit 16-18\%. Kompetenz-, Technologie- und Impulszentren sind bei 13\% der Projekte als Konsortialführung tätig. Sonstige Einrichtungen wie Vereine, Verbände oder Gemeinschaften machen einen Anteil von $3 \%$ aus.

\section{Grafik 7: Organisationsformen der Konsortialführung}

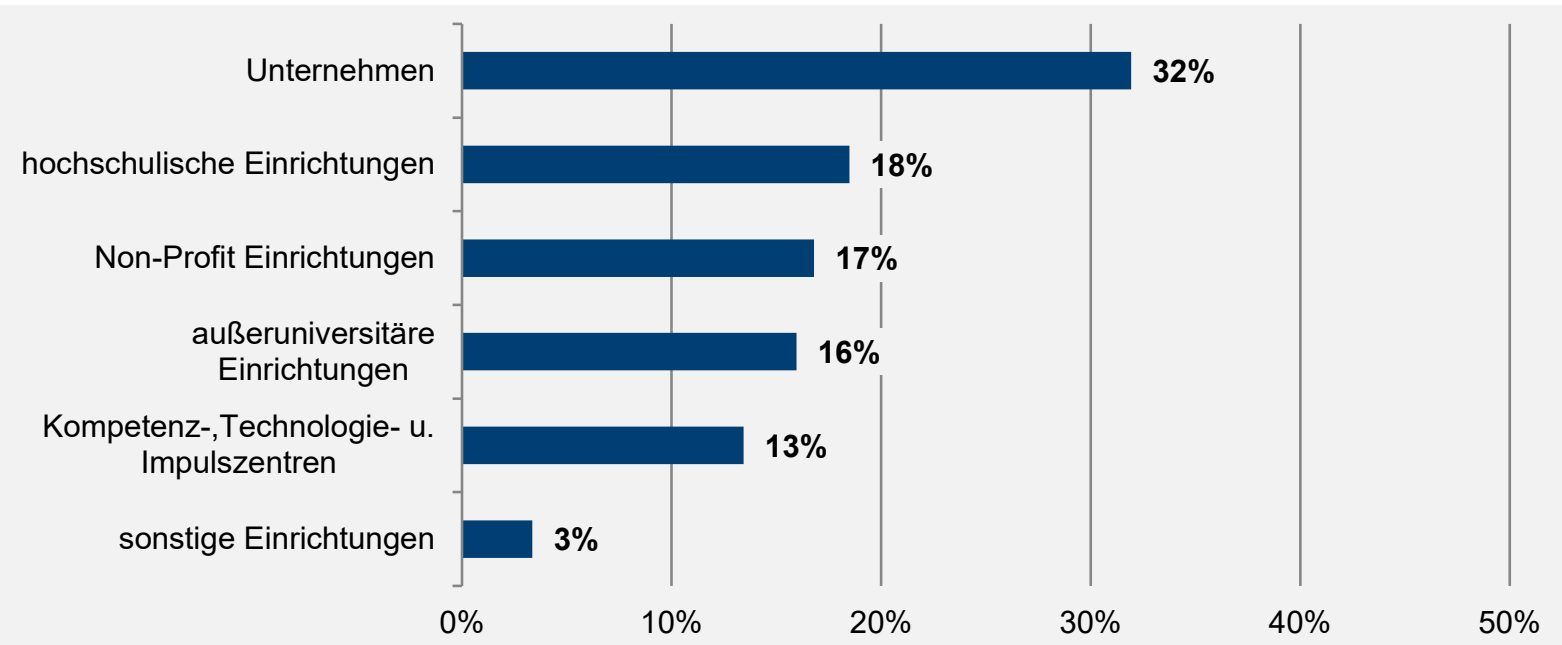

Quelle: Projektanträge und -berichte (2011-2016), n=119

Anmerkung: Bei der Kategorie „sonstige Einrichtungen“ handelt es sich um Vereine und Verbände. 
Anhand von Grafik 8 lässt sich zeigen, dass sich die Verteilung der Themenfelder zwischen den verschiedenen Ausschreibungen durchaus unterscheidet. Dies ist zu einem gewissen Grad auch auf die Besonderheiten und Schwerpunktsetzungen der einzelnen Ausschreibungen ${ }^{12}$ zurückzuführen. So ist die Ausschreibung aus dem Jahr 2011 relativ stark (über 70\%) von Projekten aus dem Bereich Energie/Umwelt und Life Sciences geprägt. Im Zeitraum zwischen 2012 und 2016 liegt der Anteil dieser beiden Bereiche dann zwischen $43 \%$ und 53\%. Der Themenbereich Mobilität ist vor allem im Jahr 2013 (25\%) und 2015 (30\%) stärker präsent. Projekte aus dem Themenbereich der Produktion machen vor allem im Jahr 2016 (26\%) einen relativ hohen Anteil aus. Außer im Jahr 2014 (24\%) liegen die sonstigen Themenbereiche zumeist bei einem Anteil zwischen $6 \%$ und $13 \%$.

Grafik 8: Themenfelder der Projekte differenziert nach Ausschreibung

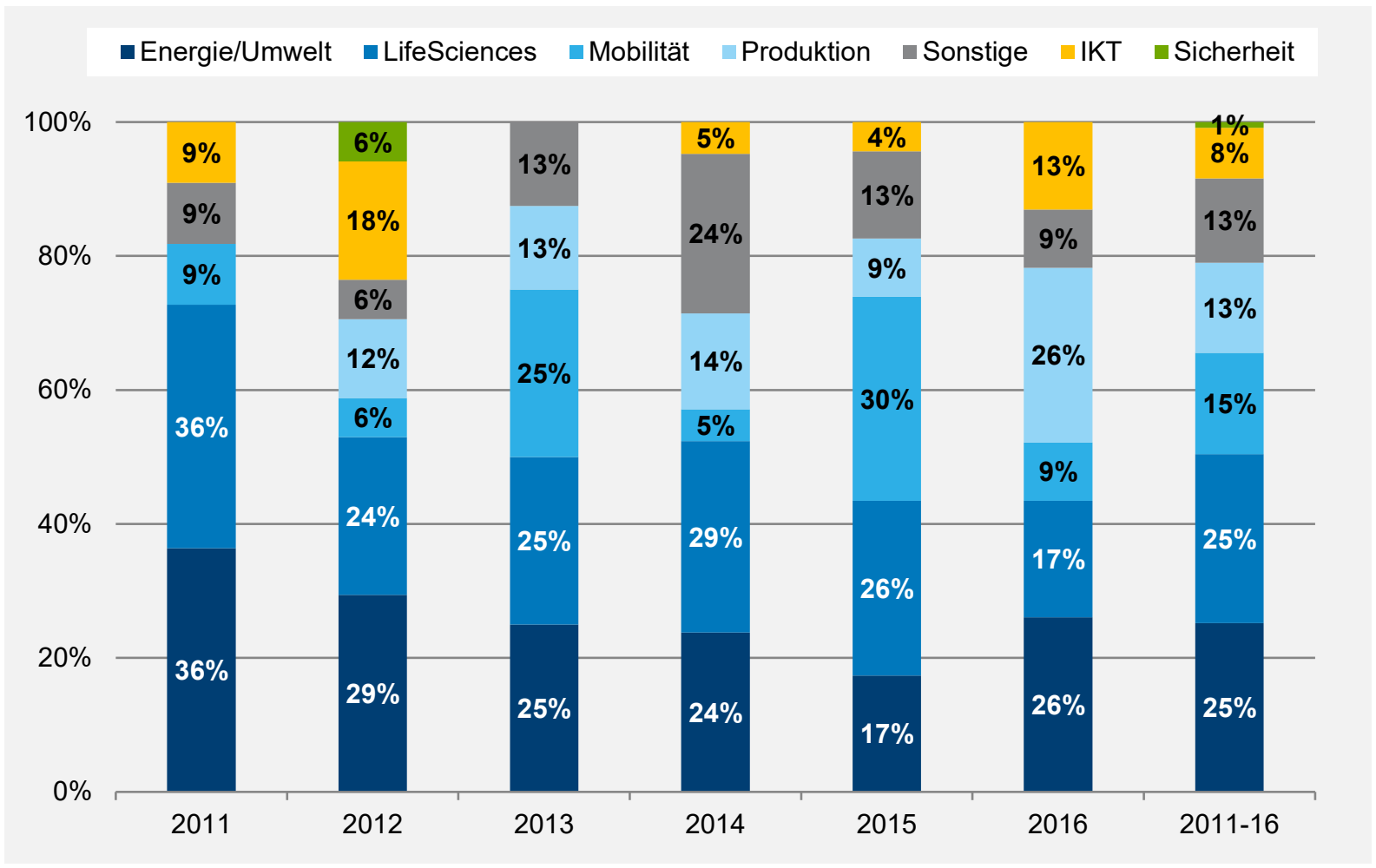

Quelle: Projektanträge und -berichte (2011-2016), n=119

\footnotetext{
12 Im Jahr 2013 (3. Ausschreibung) hat sich der Klima- \& Energiefonds (KLI.EN) an der Ausschreibung beteiligt. Es wurde ein extra KLI.EN-Budgettopf sowie ein KLI.EN-Verteiler zur Erschließung neuer Zielgruppen für Projekte aus den Themenbereichen Mobilität und Energie/Umwelt angeboten. Von den 24 Projekten wurden insgesamt acht aus KLI.EN-Mitteln gefördert.
}

Ab 2014 (4. Ausschreibung) wurde erstmals der Schwerpunkt „Migrationshintergrund“ standardmäßig in die Ausschreibung integriert, mit dem Ziel, vermehrt Kinder und Jugendliche unabhängig ihrer sozialen oder geographischen Herkunft zu berücksichtigen bzw. anzusprechen. Dieser Schwerpunkt wurde in den darauffolgenden Ausschreibungen beibehalten.

Im Jahr 2015 (5. Ausschreibung) wurde das Förderinstrument angepasst. Die maximale Förderhöhe pro Projekt wurde von EUR 60.000,- auf EUR 130.000,- angehoben und die Projektlaufzeit auf bis zu maximal 36 Monate verdoppelt. Außerdem mussten ab dieser Ausschreibung von den fünf Bildungseinrichtungen mindestens zwei Volksschulen sowie zwei Unterstufen in dem jeweiligen Projekt vertreten sein. Die fünfte Bildungseinrichtung konnte wie zuvor zwischen Kindergarten und Matura frei gewählt werden. 


\subsection{Projektbeteiligte Organisationen}

Der folgende Abschnitt stellt einzelne Charakteristika wie die Verteilung der Bundesländer, Organisationsformen und Themenfelder der am Projekt Beteiligten ${ }^{13}$ dar.

Betrachtet man die Verteilung der Organisationsformen in den Projekten (laut Projektantrag) der Ausschreibungen zwischen 2011 und 2016, so machen (vor-)schulische Bildungseinrichtungen mit insgesamt $55 \%$ den größten Anteil aus. Danach folgen Unternehmen und Forschungseinrichtungen mit $22 \%$ bzw. $17 \%$. Vereine, Länder oder Gemeinden machen lediglich einen Anteil von $7 \%$ der Projektbeteiligten aus.

\section{Grafik 9: Organisationsformen der Projektbeteiligten}

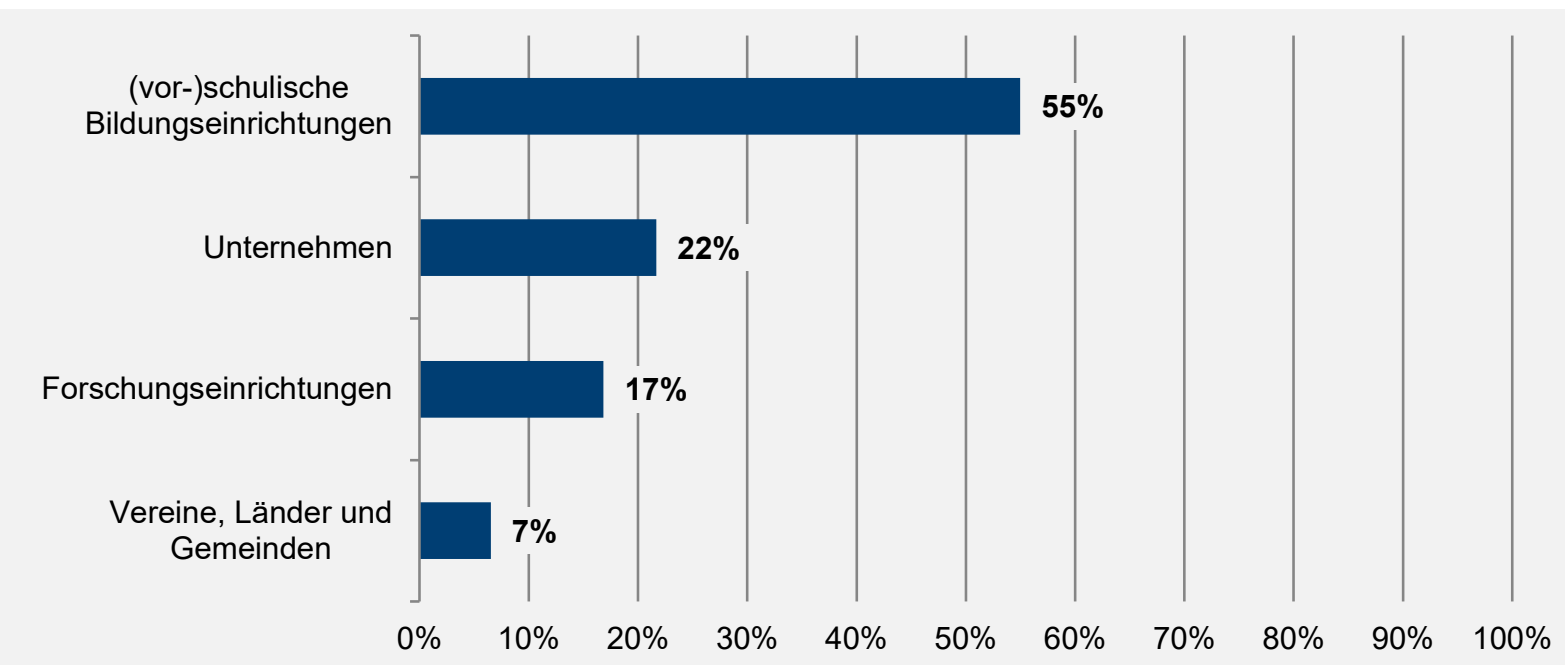

Quelle: Projektanträge und -berichte (2011-2016), n=1.366

Anmerkung: Da in den Projektberichten aus dem Jahr 2010 keine systematisierten Informationen zu den Organisationsformen der Konsortialführung und den Themenfeldern der Projekte vorhanden sind, beziehen sich Grafik 9 und Grafik 10 auf Projekte ab der Ausschreibung aus dem Jahr 2011.

Die detaillierte Auswertung der Organisationsformen in Grafik 10 zeigt, dass die Volksschulen mit $17 \%$ den größten Anteil ausmachen. Danach folgen die Neue Mittelschule/Hauptschule und die Allgemeinbildende Höhere Schule mit $12 \%$ bzw. 10\%. Berufsbildende Höhere Schulen haben einen Anteil von $7 \%$. Die Polytechnische Schule sowie sonstige Bildungseinrichtungen machen $1 \%$ bzw. $2 \%$ aus. Die Verteilung der Schulen ist stark von den jeweiligen Ausschreibungskriterien der verschiedenen Ausschreibungen geprägt.

Bei den Forschungseinrichtungen dominieren die Universitäten und Fachhochschulen mit 12\%. Außeruniversitäre und sonstige Forschungseinrichtungen machen lediglich $4 \%$ bzw. $1 \%$ aus. Bei den Vereinen machen wissenschaftsorientierte bzw. nicht wissenschaftsorientierte jeweils $3 \%$ aus und Länder und Gemeinden lediglich $1 \%$.

Bei den beteiligten Unternehmen überwiegen kleinere Unternehmen, welche insgesamt $12 \%$ aller Organisationsformen ausmachen. Mittelgroße bzw. große Unternehmen haben einen Anteil von insgesamt $4 \%$ bzw. $6 \%{ }^{14}$.

13 Es handelt sich dabei um das Projektkonsortium (Konsortialführung, wissenschaftliche Partnerlnnen und Unternehmenspartnerlnnen) und Bildungseinrichtungen, die in Form einer Projektbeteiligung vertreten waren. Nicht berücksichtigt wurden Bildungseinrichtungen, die einen Kooperationszuschuss erhalten haben. 


\section{Grafik 10: Verteilung der Organisationsformen im Detail}

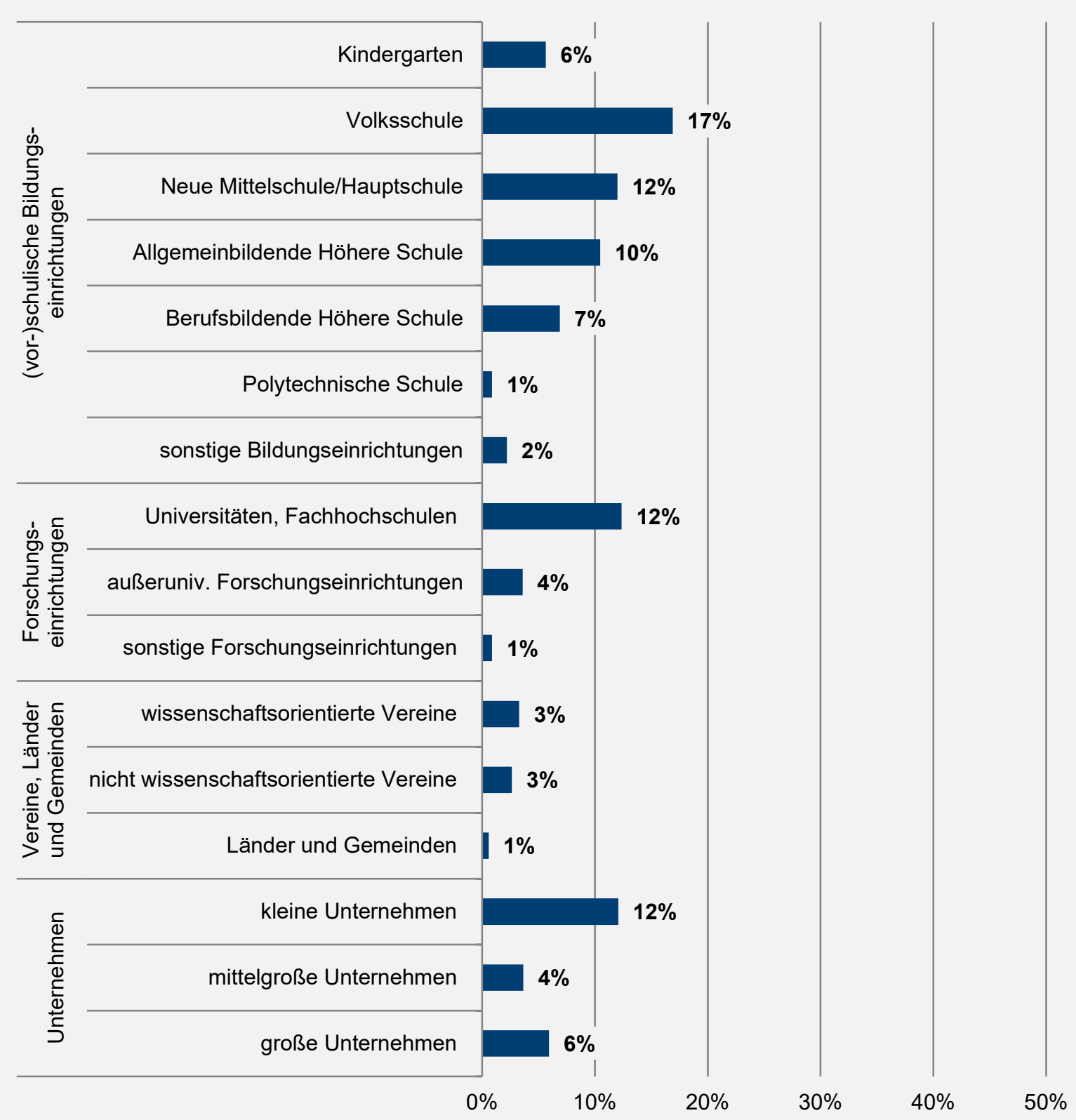

Quelle: Projektanträge und -berichte (2011-2016), n=1.366

Anmerkung: Bei den sonstigen Bildungseinrichtungen handelt es sich bspw. um Waldorfschulen, Montessorischulen sowie Mischformen an einem Standort.

\footnotetext{
14 Die Kategorisierung der Unternehmensgrößen basiert auf einer Empfehlung der Kommission der europäischen Gemeinschaft vom 6.5.2003 betreffend die Definition der Kleinstunternehmen sowie der kleinen und mittleren Unternehmen (URL: https://eur-lex.europa.eu/legal-content/DE/TXT/PDF/?uri=CELEX:32003H0361\&...). Bezüglich der Beschäftigtenzahlen (Vollzeitäquivalent) werden Unternehmen mit weniger als 50 Beschäftigten als „kleine Unternehmen“ und jene mit mehr als 50 und weniger als 250 Beschäftigten als „mittelgroße Unternehmen“ bezeichnet. Betriebe mit mehr als 250 Beschäftigten fallen in die Kategorie "große Unternehmen“. Zusätzlich werden auch noch finanzielle Schwellenwerte zur Definition der Unternehmensklassen herangezogen.
} 
Die grobe Zusammensetzung der Organisationsformen der Projektbeteiligten unterscheidet sich zwischen den einzelnen Ausschreibungen nur marginal und bleibt im Zeitverlauf äußerst stabil. Dies hängt mit den gleichbleibenden Ausschreibungskriterien zusammen.

\section{Grafik 11: Verteilung der Organisationsformen differenziert nach Ausschreibung}

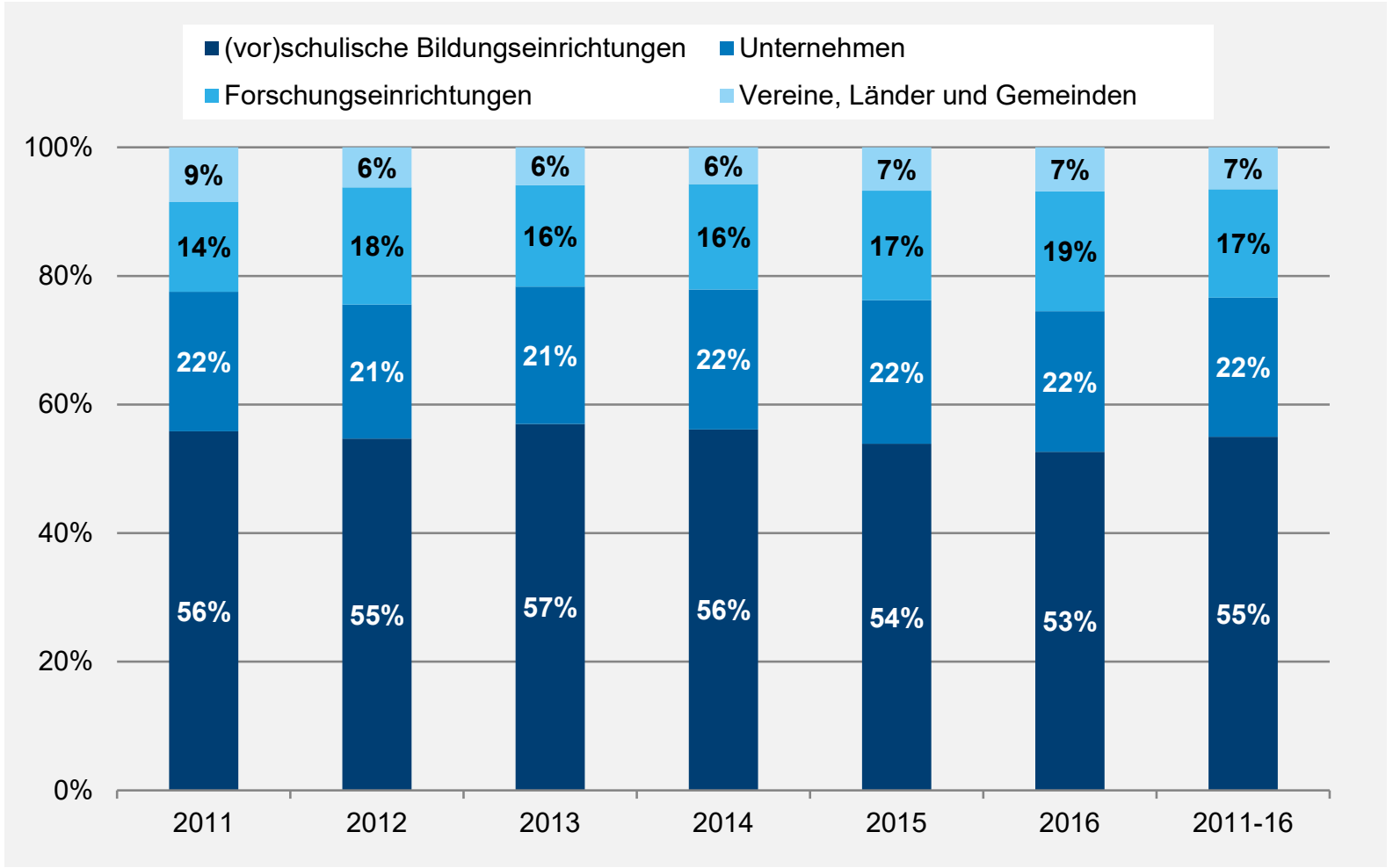

Quelle: Projektanträge und -berichte (2011-2016), n=1.366 


\subsection{Kooperationszuschüsse}

Der folgende Abschnitt vergleicht nun die einzelnen Ausschreibungen in Bezug auf die Kooperationszuschüsse. Dabei handelt es sich um Pauschalförderungen für Bildungseinrichtungen, die nicht am Projekt beteiligt sind, um die Bekanntheit von „Talente regional“ zu verbessern und innovative Unterrichtsprojekte im Bereich Naturwissenschaft und Technik an Kindergärten und Schulen zu fördern.

Zur Analyse werden die Anzahl der bearbeiteten Anträge, die förderbare Maximalanzahl sowie die genehmigten Anträge gegenübergestellt. Auf Basis dieser Daten können dann auch Quoten zur Ausschöpfung der Kooperationszuschüsse errechnet werden.

Bei der Ausschreibung aus dem Jahr 2010 gab es noch keine Kooperationszuschüsse, weswegen dieses Jahr nicht in die Auswertung aufgenommen wurde. Auch das Jahr 2016 wurde nicht aufgenommen, da hier noch keine ausreichende Datenbasis zur Verfügung steht. Anzumerken ist, dass die Daten aus dem Jahr 2015 auf den Zwischenberichten basieren und sich somit noch ändern werden.

Wie die Grafik 12 zeigt, gab es lediglich in den Jahren 2011 und 2012 mehr Anträge als Kooperationszuschüsse. Von 2013 bis 2015 lag die Anzahl der bearbeiteten Anträge auf Kooperationszuschüsse unter dem maximalen Kontingent.

\section{Grafik 12: Anzahl der Kooperationszuschüsse}

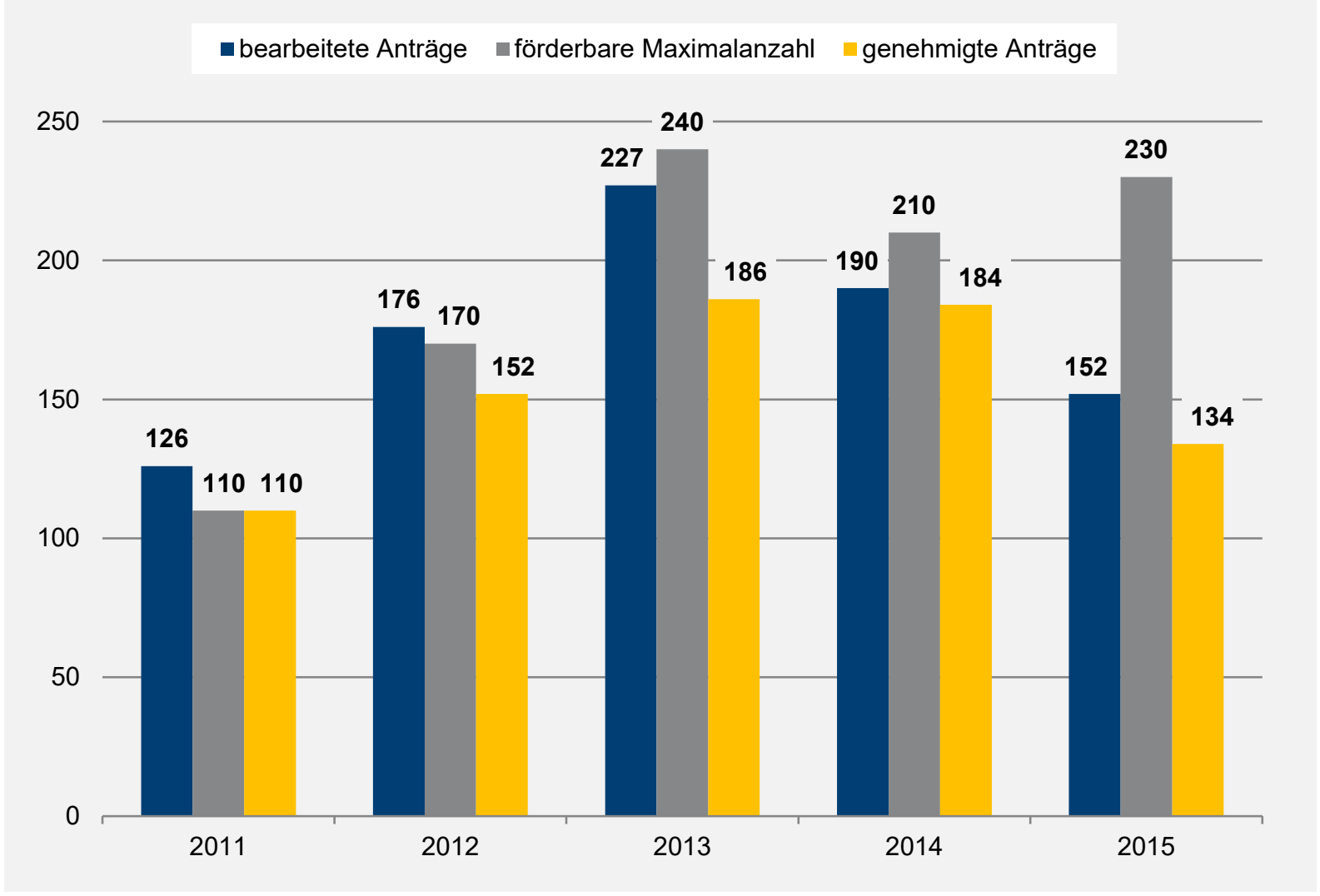

Quelle: Projektanträge und -berichte (2011-2015)

Anmerkung: Die Daten aus dem Jahr 2015 basieren auf Zwischenberichten. 
Gänzlich ausgeschöpft wurde die maximale Anzahl an Kooperationszuschüssen nur im Jahr 2011. Auch in den Jahren 2012 und 2014 ist die Inanspruchnahme der Kooperationszuschüsse mit 89\% bzw. 88\% relativ hoch. Unabhängig vom Jahr 2015, wo noch keine Daten aus Endberichten vorliegen, ist der Grad der Ausschöpfung mit 78\% im Jahr 2013 am geringsten (vgl. Grafik 13).

Der Anteil der genehmigten Kooperationszuschüsse an den bearbeiteten Anträgen ist im Jahr 2014 mit $97 \%$ besonders hoch. In den restlichen Jahren liegt der Wert zwischen $82 \%$ und $88 \%$.

\section{Grafik 13: Ausschöpfungsgrade der Kooperationszuschüsse}

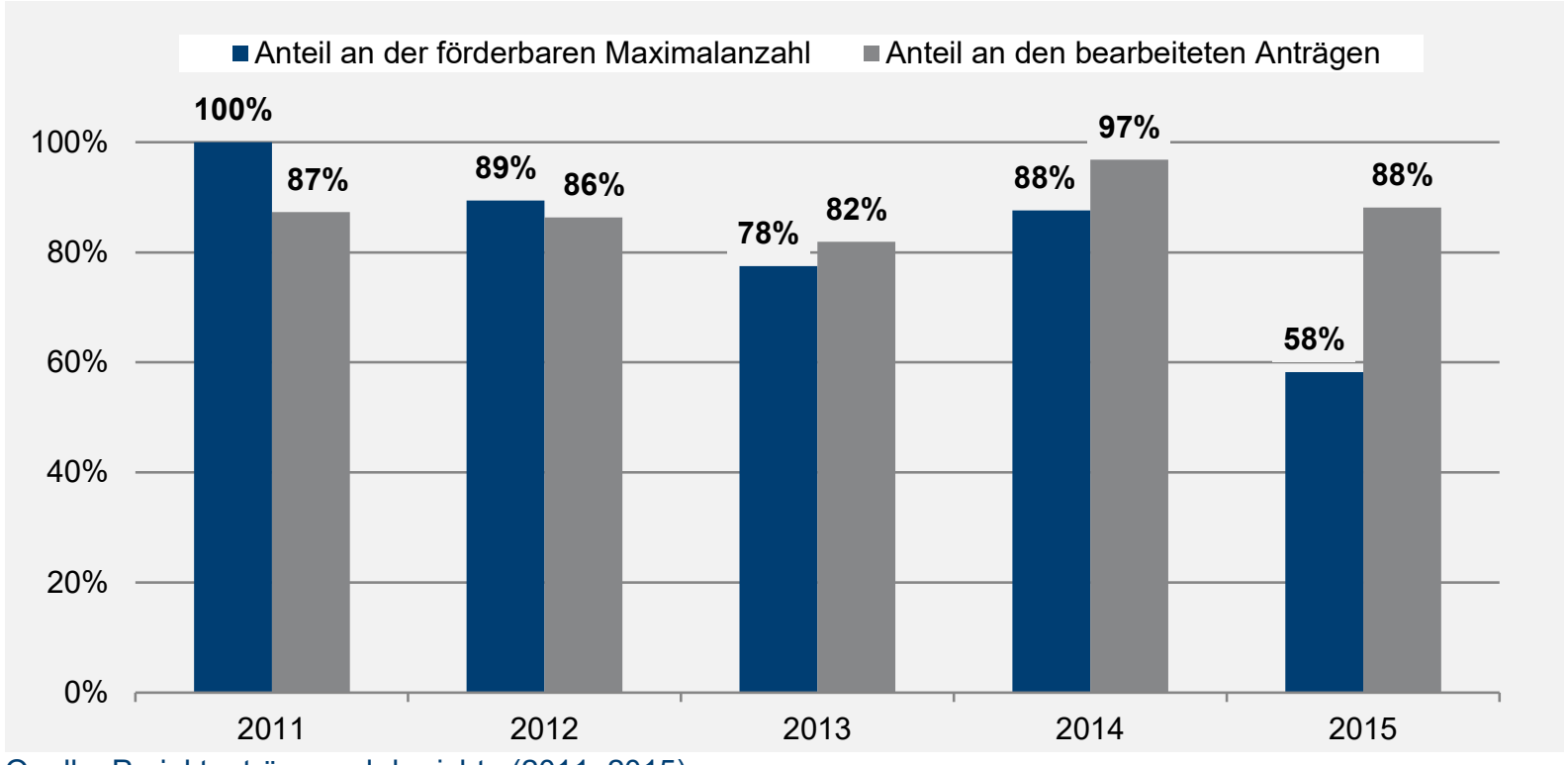

Quelle: Projektanträge und -berichte (2011-2015)

Anmerkung: Die Daten aus dem Jahr 2015 basieren ausschließlich auf Zwischenberichten.

Diese hohen Ausschöpfungsgrade unterstreichen einerseits die Attraktivität der Kooperationszuschüsse und andererseits die hohe Bedeutung zur Stärkung der Bekanntheit von „Talente regional“. Offenbar ist sowohl das Interesse, als auch die Bereitschaft an den Kindergärten und Schulen eigene lokale Aktivitäten und Maßnahmen durchzuführen bzw. aufzubauen überaus hoch. 


\subsection{Eingebundene Kinder und Jugendliche}

Der folgende Abschnitt vergleicht die einzelnen Ausschreibungen in Bezug auf die in die Projektaktivitäten ${ }^{15}$ eingebundenen Kinder und Jugendliche. Da zum Zeitpunkt der Auswertungen noch keine Endberichte aus dem Jahr 2015 (5. Ausschreibung) und keine Zwischenberichte aus dem Jahr 2016 (6. Ausschreibung) zur Verfügung standen, beziehen sich die Grafiken auf den Zeitraum zwischen 2010 und 2014. In Grafik 16 konnte außerdem das Jahr 2010 aufgrund der fehlenden Informationen zu den Themenfeldern der Projekte nicht berücksichtigt werden.

Anhand der Darstellung in Grafik 14 zeigt sich, dass die Projekte aus dem Jahr 2010 im Durchschnitt die größte Anzahl an Kindern und Jugendlichen adressiert haben. In den darauf folgenden Jahren sind die Werte wesentlich geringer und liegen zwischen 312 und 414 Personen. Der Durchschnitt über alle Ausschreibungen zwischen 2010 und 2014 liegt bei 388.

Grafik 14: Durchschnittliche Anzahl der in die Projektaktivitäten eingebundenen Kinder/Jugendlichen pro Projekt

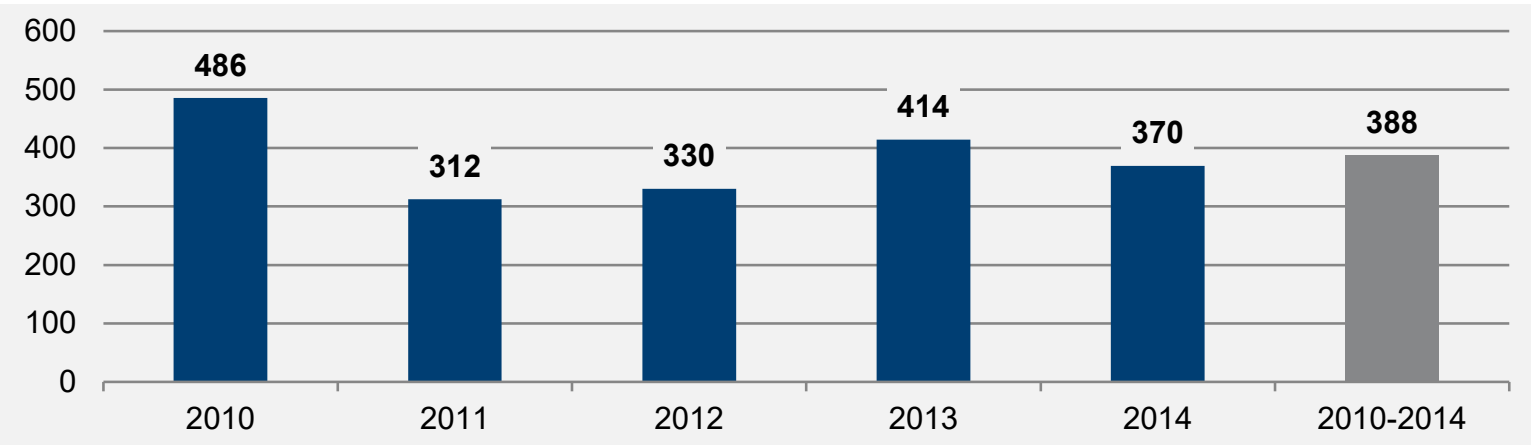

Quelle: Projektanträge und -berichte (2010-2014)

Die Gegenüberstellung der Anteile der Geschlechter illustriert die Ausgewogenheit der Aktivitäten in den verschiedenen Ausschreibungen. Lediglich bei den Projekten aus der Ausschreibung im Jahr 2010 liegt der Anteil der Burschen über jenem der Mädchen.

\section{Grafik 15: Verteilung der Geschlechter der in die Projektaktivitäten eingebundenen Kin- der/Jugendlichen}

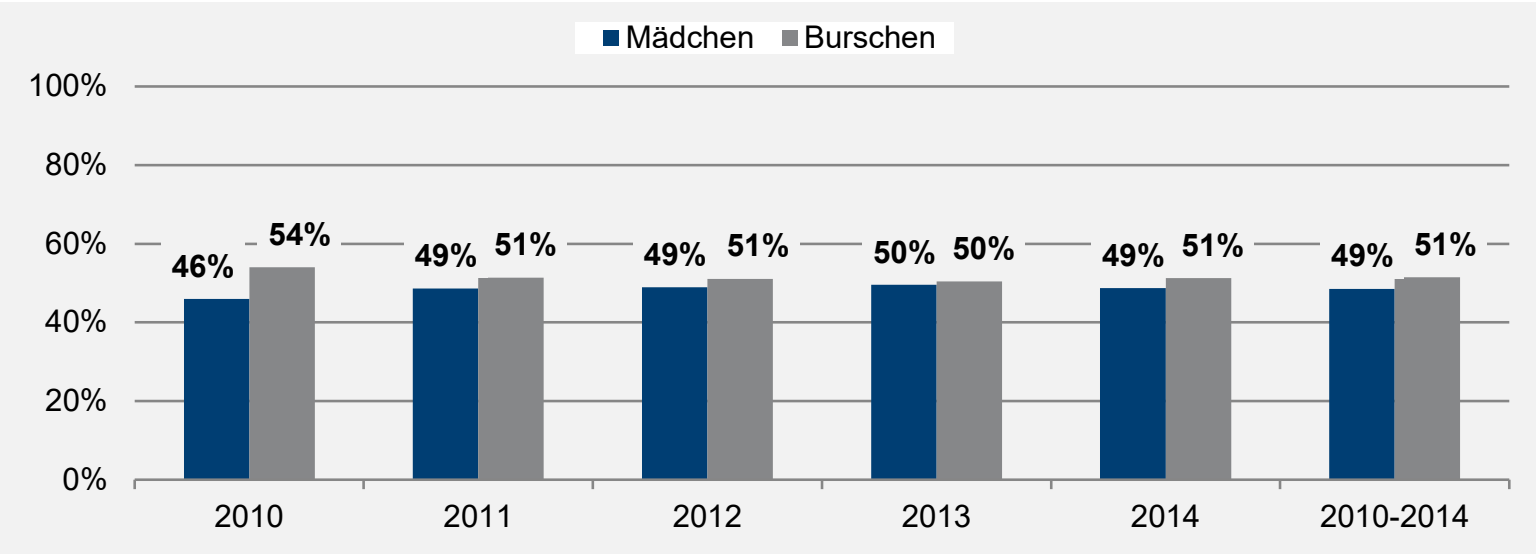

Quelle: Projektanträge und -berichte (2010-2014)

Anmerkung: Die Angaben von zwei Projekten $(2010,2013)$ konnten aufgrund fehlender Werte zur Geschlechterverteilung in dieser Darstellung nicht berücksichtigt werden.

${ }^{15}$ Aktivitäten im Rahmen der Kooperationszuschüsse wurden nicht berücksichtigt. 
Vergleicht man die Verteilung der Projekte mit den damit erreichten Kinder und Jugendlichen nach Themenfeldern, zeigt sich eine hohe Übereinstimmung. Lediglich im Bereich Energie/Umwelt konnten überdurchschnittlich viele Kinder und Jugendliche angesprochen werden. Bei den „sonstigen Themenfeldern" wurden hingegen weniger Personen erreicht. Bei allen anderen Themenfeldern gibt es nur marginale Differenzen (vgl. Grafik 16).

Grafik 16: Vergleich der Verteilungen auf die Themenfelder (2011-2014)

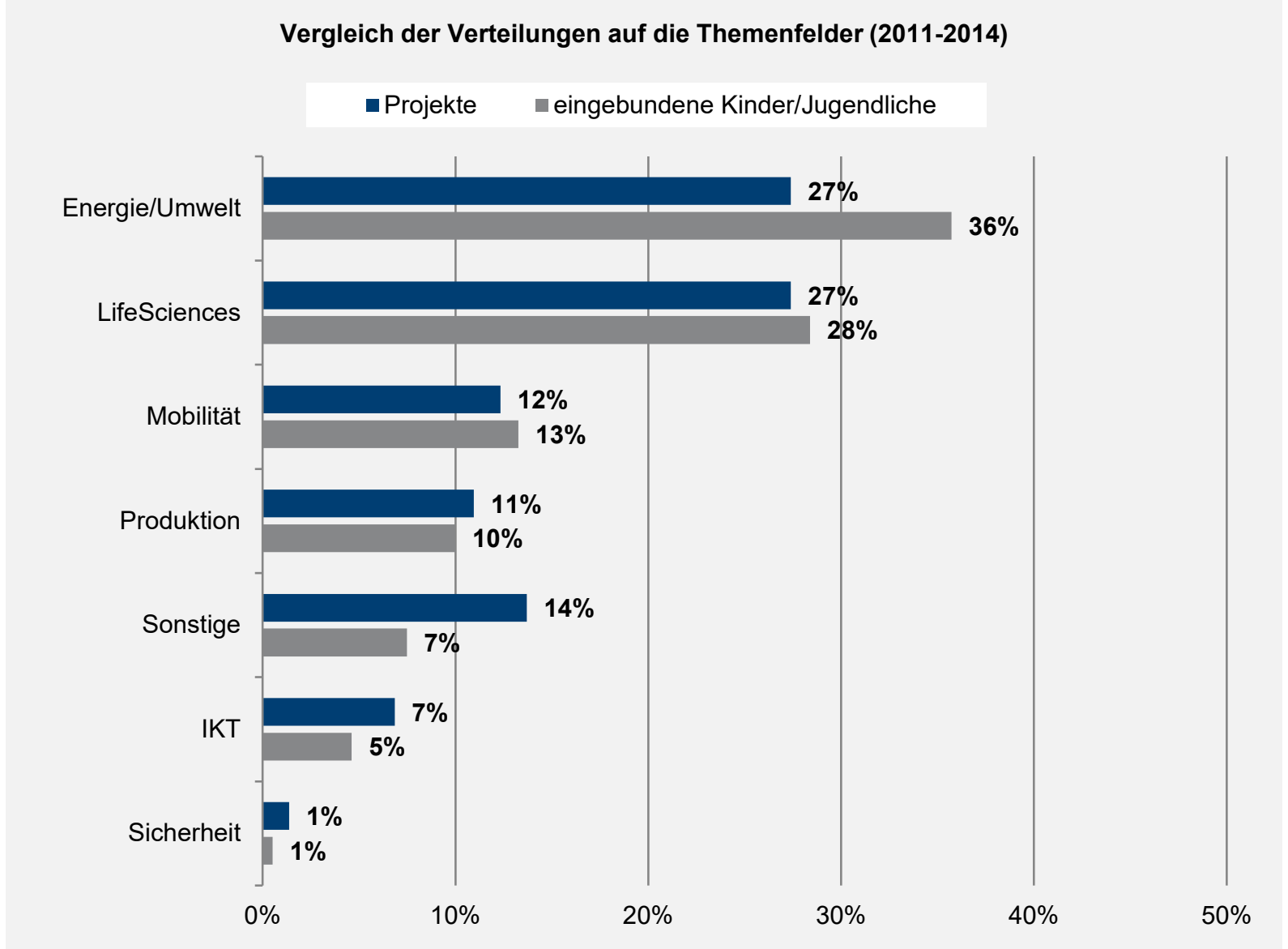

Quelle: Projektanträge und -berichte (2011-2014), n=73 (Projekte), n=26.753 (eingebundene Kinder/Jugendliche) 


\subsection{Bundesländervergleich}

Grafik 17 offenbart, dass die relativen Verteilungen differenziert nach Bundesländern zwischen den Projekten (Konsortialführung) und den Projektbeteiligten sehr ähnlich sind. Lediglich in Wien waren verhältnismäßig weniger Organisationen an den verschiedenen Projekten beteiligt.

Bezogen auf die relative Verteilung der eingebundenen Zielgruppen sind die Unterschiede in den einzelnen Bundesländern wesentlich größer. In den Bundesländern Burgenland, Niederösterreich und Tirol wurden verhältnismäßig weniger Kinder und Jugendliche in die Projekte eingebunden. In Oberösterreich hingegen wurden offenbar überproportional viele Kinder und Jugendliche erreicht. In den restlichen Bundesländern unterschieden sich die Anteile nur in einem geringeren Maße.

Grafik 17: Vergleich der Verteilungen auf die Bundesländer (2010-2014)

Vergleich der Verteilungen auf die Bundesländer (2010-2014)

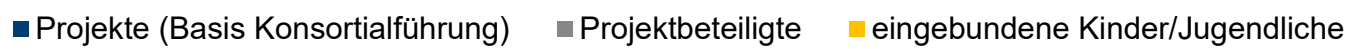

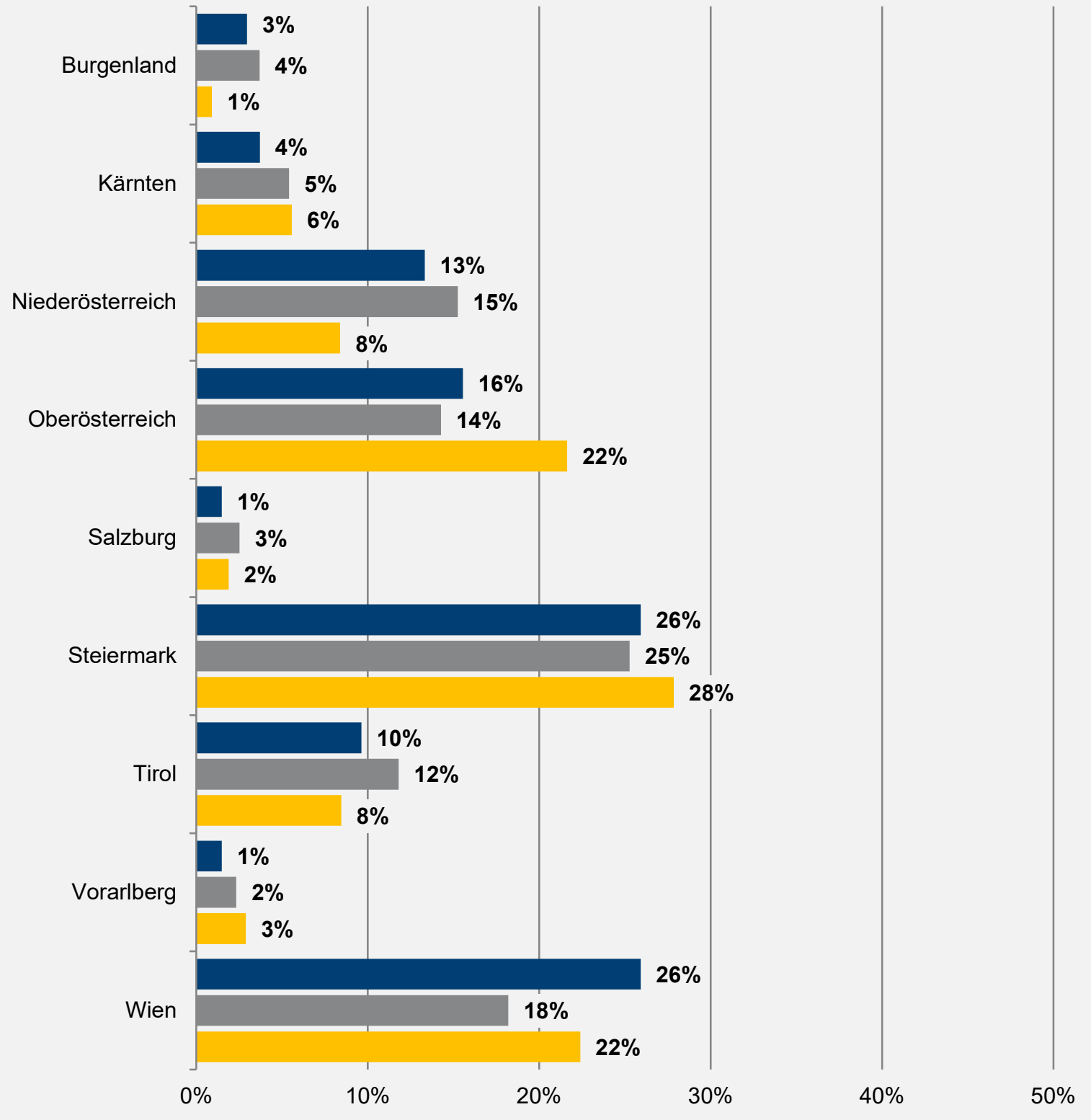

Quelle: Projektanträge und -berichte (2010-2014) 


\section{Quantitative Befragung der Projektbeteiligten}

Um die Gemeinsamkeiten, aber auch allfällige Unterschiede in den Bewertungen der Projektbeteiligten auf einer repräsentativen Basis erfassen zu können, wurde eine Online-Befragung durchgeführt. Zentrale Themenfelder waren neben der Zufriedenheit mit der Projektabwicklung und -gestaltung vor allem die Vereinbarkeit der Ausschreibungsschwerpunkte, Erfolgsfaktoren und Wirkungen der Projektaktivitäten und Kooperationszuschüsse, sowie die Nachhaltigkeit der Kooperationen.

Als Basis für die Kontaktaufnahme standen die E-Mail-Adressen der Konsortialführungen zur Verfügung. Diese wurden von der FFG dazu eingeladen, an der Befragung teilzunehmen. Außerdem wurden sie aufgefordert, dieses E-Mail mit dem Link zur Befragung an andere am Projekt Beteiligte weiterzuleiten und sich auch an der Befragung zu beteiligen. Die Unterstützung der Konsortialführungen war somit eine wesentliche Voraussetzung, um die Perspektive des gesamten Projektkonsortiums (wissenschaftliche PartnerInnen, UnternehmenspartnerInnen) und vor allem der vielen beteiligten Bildungseinrichtungen (auch jene mit Kooperationszuschuss) erfassen zu können.

Von den 135, in den Jahren 2010 bis 2016 geförderten, Projekten waren insgesamt $106^{16}$ Kontaktadressen vorhanden. $96^{17}$ konnten per E-Mail erreicht werden. Der Befragungszeitraum erstreckte sich vom 15. Jänner bis zum 16. Februar 2018. Außerdem wurde zusätzlich zur Einladung am Beginn der Befragung am 31. Jänner 2018 ein Reminder verschickt.

Insgesamt 245 RespondentInnen haben sich an der Befragung beteiligt, wovon allerdings 64 den Fragebogen bereits frühzeitig abgebrochen haben. Von den 181 RespondentInnen, die zur Auswertung herangezogen wurden, handelt es sich bei 63 um Konsortialführungen. Bezogen auf die Anzahl der erreichten bzw. vorhandenen Kontakte entspricht dies einer Rücklaufquote von $\mathbf{6 6 \%}$ und einem Abdeckungsgrad von $59 \%$.

Eine Berechnung der Rücklaufquote auf Basis aller adressierten RespondentInnen ist aufgrund der indirekten Kontaktaufnahme der Projektbeteiligten (außer der Konsortialführung) nicht möglich. Da im Fragebogen allerdings nach der Anzahl der Projekteilnahmen gefragt wurde, kann diese Summe (329 Projektbeteiligungen) in Relation zur Gesamtanzahl der Projektbeteiligten (vgl. Abbildung 1, 1.626 Projektbeteiligte) aus der deskriptiven Projektübersicht gestellt werden. Der Abdeckungsgrad der Befragung für alle an den Projekten Beteiligte liegt bei $20 \%$.

Da bei allen Projektbeteiligten ein gewisser Anteil an Organisationen schon mehrmals an Projekten teilgenommen hat, wurden die RespondentInnen in der Befragung darum gebeten, nur auf die letzte (aktuellste) Teilnahme Bezug zu nehmen.

\footnotetext{
${ }^{16}$ Der Anteil der Konsortialführungen, die mehrere Projekte durchgeführt haben, liegt bei $21 \%$.

17 Der Anteil der Kontakte, die nicht erreicht wurden bzw. wo die E-Mail unzustellbar war, liegt bei $9 \%$.
} 


\subsection{Charakteristika der Stichprobe}

Rund zwei Drittel der RespondentInnen gaben an, dass sie zum Zeitpunkt der Befragung an einem Projekt (im Rahmen von "Talente regional“ bzw. "generation innovation Regionen“) beteiligt waren. Etwas mehr als ein Drittel weist zumindest zwei Projektbeteiligungen auf. Ein Zehntel der RespondentInnen hat sogar mehr als drei Mal in Projekten mitgewirkt (vgl. Grafik 18).

\section{Grafik 18: Anzahl der Projektbeteiligungen}

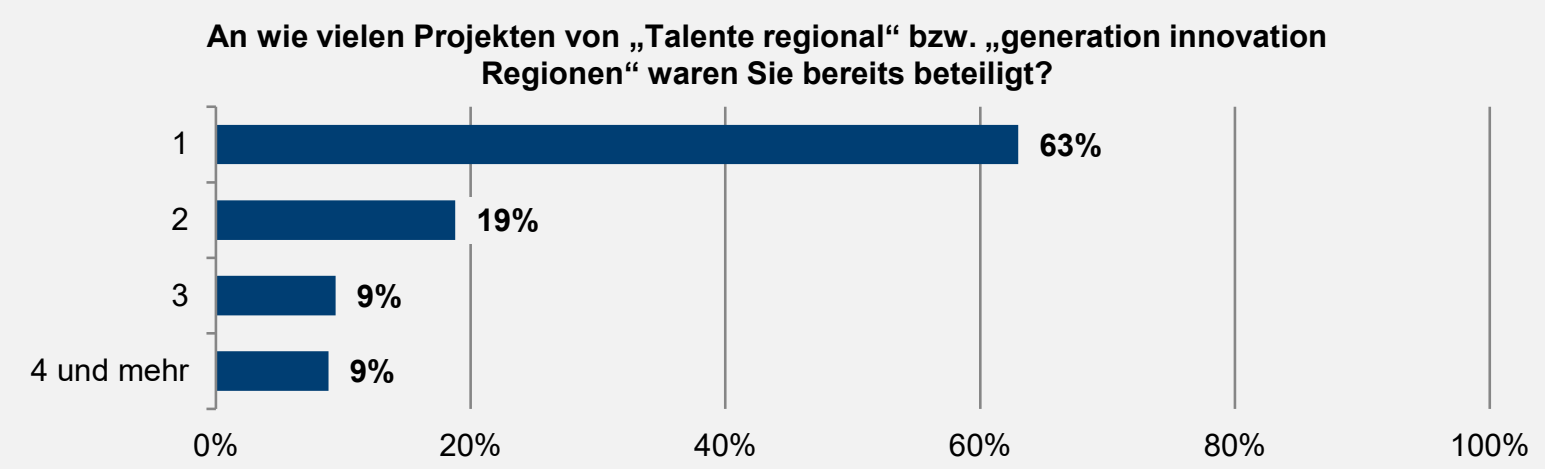

Quelle: ibw-Befragung 2017, n=181

Der Großteil der Befragten, über drei Viertel, gibt an, dass sich ihre Angaben auf eine der letzten drei Ausschreibungen (2014 bis 2016) beziehen. Die letzte Ausschreibung aus dem Jahr 2016 ist mit fast $40 \%$ besonders stark vertreten (vgl. Grafik 19 ).

Diese Verteilung des Rücklaufs ist einerseits von der Tatsache geprägt, dass die Befragten, welche bereits an mehreren Projekten teilgenommen haben, gebeten wurden, nur auf die letzte (aktuellste) Teilnahme Bezug zu nehmen. Und andererseits liegt der Schluss nahe, dass es bei den Projekten aus älteren Ausschreibungen zu Veränderungen beim Personal bzw. den Kontaktdaten gekommen ist.

\section{Grafik 19: Projektbezug der Antworten}

Die Antworten im Rahmen dieser Befragung beziehen sich auf ein Projekt aus der:

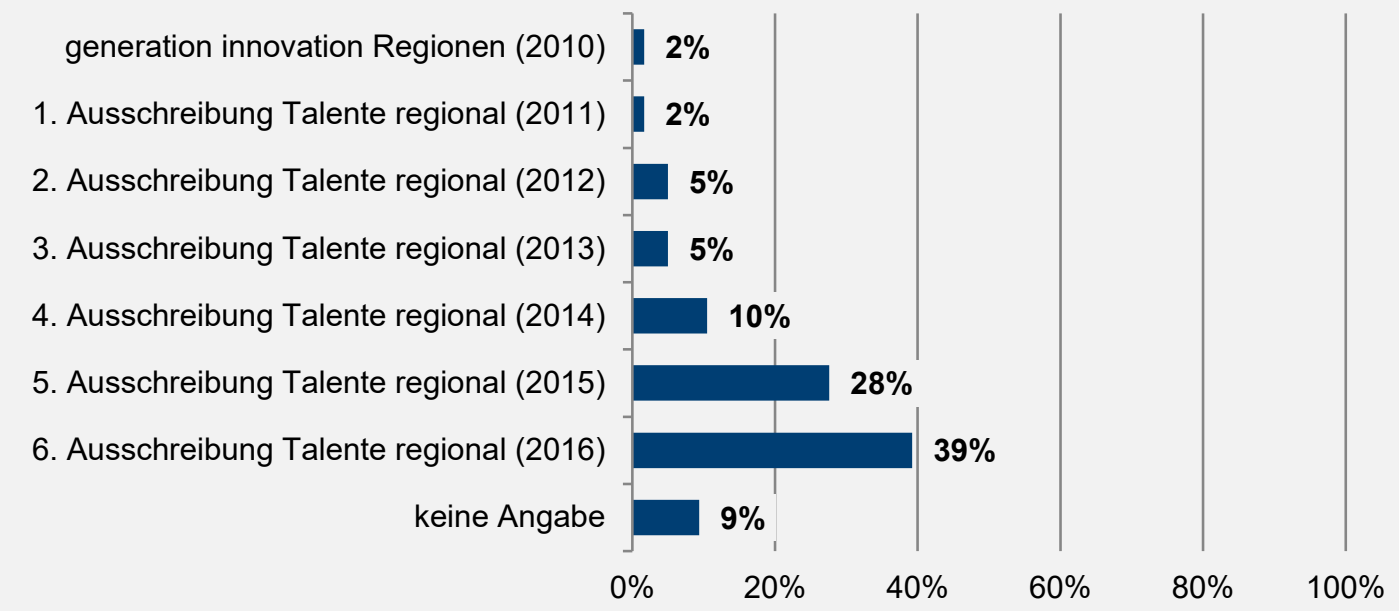

Quelle: ibw-Befragung 2017, n=181

Bei den Rollen, die die Befragten im jeweiligen Projekt übernommen haben, entfällt über ein Drittel der Antworten auf die Konsortialführung. Danach folgen die (vor-)schulischen Bildungseinrichtungen als Projektbeteiligte mit $20 \%$ und jene mit Kooperationszuschuss mit $15 \%$. Organisationen, welche die wissenschaftliche bzw. unternehmerische Rolle als Projektpartnerln übernommen haben, machen $14 \%$ bzw. $12 \%$ aus (vgl. Grafik 20 ). 


\section{Grafik 20: Rolle im Projekt}

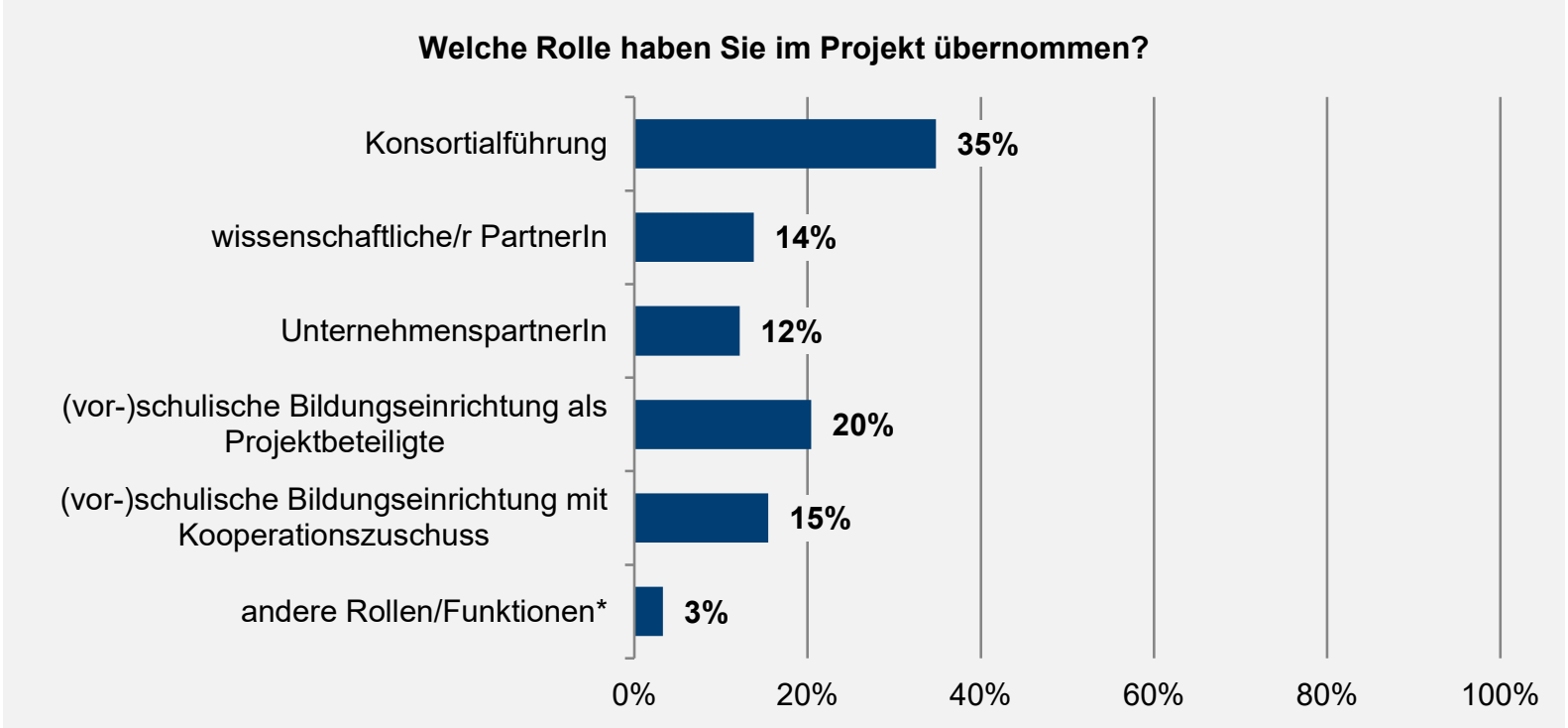

Quelle: ibw-Befragung 2017, n=181

Anmerkung: Unter die Kategorie „andere Rollen/Funktionen“ fallen sogenannte „assoziierte PartnerInnen“, die im Projekt eine unterstützende Rolle eingenommen, aber keine Kosten geltend gemacht haben.

Die Verteilung der Organisationsformen ist mit $42 \%$ besonders stark von den (vor-)schulischen Bildungseinrichtungen geprägt. Darauf folgen die Unternehmen mit einem Anteil von $20 \%$ und die hochschulischen Einrichtungen mit 14\%. Die sonstigen Formen, bei denen es sich vor allem um Vereine und Verbände handelt, machen 12\%, und die außeruniversitären Forschungseinrichtungen $9 \%$ aus (vgl. Grafik 21).

\section{Grafik 21: Organisationsformen}

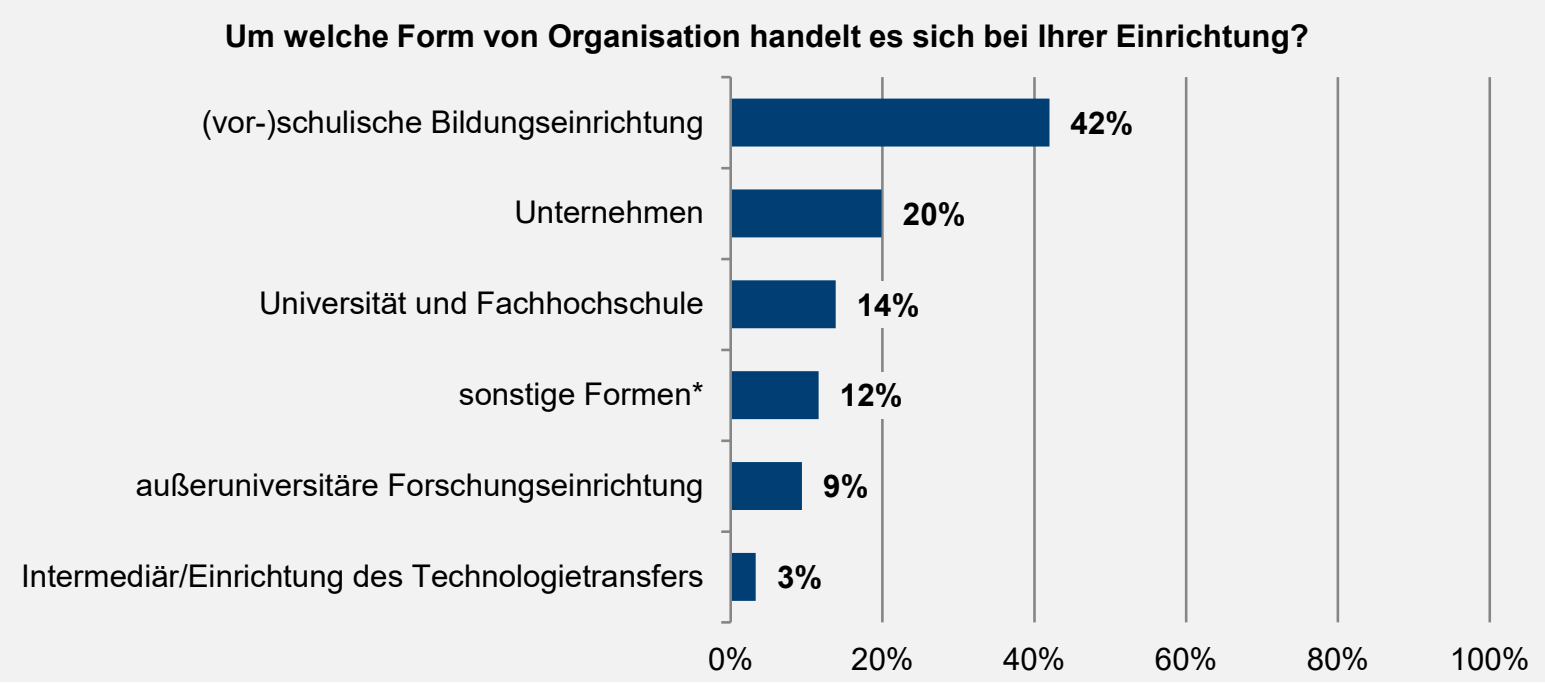

Quelle: ibw-Befragung 2017, n=181

Anmerkung: Unter die Kategorie „sonstige Formen“ fallen Einrichtungen wie Vereine bzw. Verbände.

Bei den Bildungseinrichtungen, die an der Befragung teilgenommen haben, macht die Volksschule mit $41 \%$ den größten Anteil aus (vgl. Grafik 22). An zweiter Stelle kommen die neuen Mittelschulen (Hauptschulen) mit 20\%. Der Anteil der allgemeinbildenden höheren Schule liegt in der Unterstufe bei $11 \%$ und in der Oberstufe bei $13 \%$. Auf dem gleichen Niveau liegen die berufsbildenden mittleren und höheren Schulen. Sonstige Formen wie Sonderschulen oder alternative Schulprojekte machen 7\% aus. Somit ähnelt die Grobstruktur der an der Befragung teilgenommenen Bildungseinrichtungen jener der Grundgesamtheit (vgl. Grafik 10). 


\section{Grafik 22: Formen der Bildungseinrichtungen}

Welcher dieser Formen kann Ihre Organisation zugeordnet werden? (Mehrfachantworten möglich)

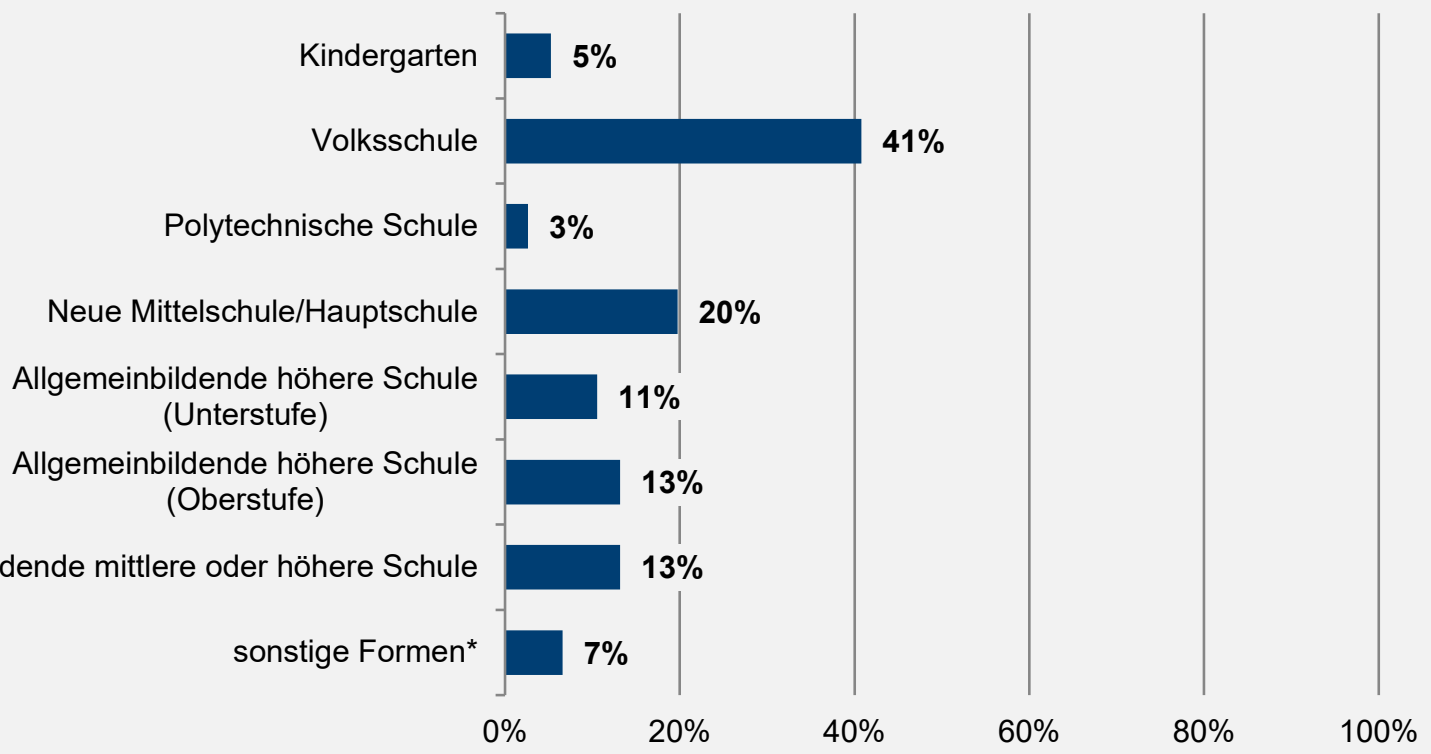

Quelle: ibw-Befragung 2017, n=76 (Mehrfachnennungen)

Anmerkung: Unter die Kategorie „sonstige Formen“ fallen Formen wie Sonderschulen oder alternative Schulprojekte.

Bei den Unternehmen, die sich an der Befragung beteiligt haben, machen Ingenieurbüros mit $20 \%$ den größten Anteil aus. Danach folgen mit jeweils 11\% Unternehmen aus den Bereichen Informationstechnologie, Produktion und Forstwirtschaft. Aber auch dem Bereich Medizintechnik, Lebensmittel und Umwelt können jeweils 9\% der Unternehmen zugeordnet werden. An letzter Stelle kommen die Unternehmensberatungen mit $6 \%$. Erwartungsgemäß haben also überwiegend Unternehmen aus naturwissenschaftlichen und technischen Bereichen teilgenommen (vgl. Grafik 23).

\section{Grafik 23: Branchen der Unternehmen}

In welcher Branche ist Ihr Unternehmen hauptsächlich tätig?

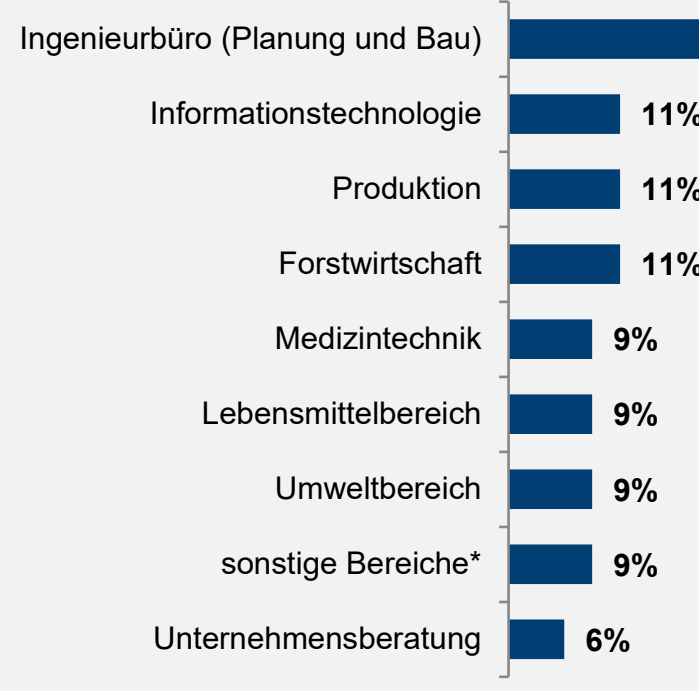

$0 \% \quad 20 \%$
$20 \%$

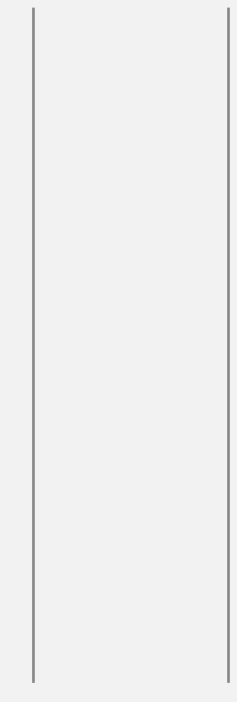

$60 \%$

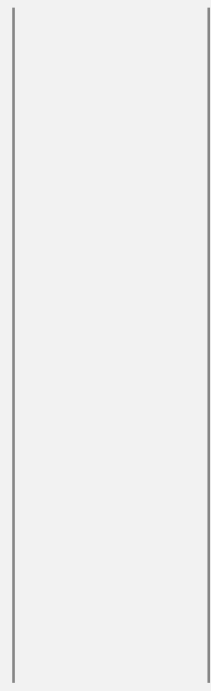

$80 \%$

$100 \%$

Quelle: ibw-Befragung 2017, n=35

Anmerkung: Unter die Kategorie „sonstige Bereiche“ fallen Nennungen wie Chemie, Forschung oder Regionalentwicklung und Innovationsförderung. 
Diese Unternehmen zeichnen sich zudem durch einen hohen Anteil an Beschäftigten in „MINTBerufen"18 aus. Fast drei Viertel der RespondentInnen gaben an, dass ihre Belegschaft zu über $50 \%$ diesem Berufsfeld zugeordnet werden kann und bei rund der Hälfte der Unternehmen liegt dieser Anteil sogar über $75 \%$ (vgl. Grafik 24).

\section{Grafik 24: MINT-Orientierung der Unternehmen}

Bitte schätzen Sie den Anteil der Beschäftigten in Ihrem Unternehmen, die dem Berufsfeld der „MINT-Berufe“ zugeordnet werden können.

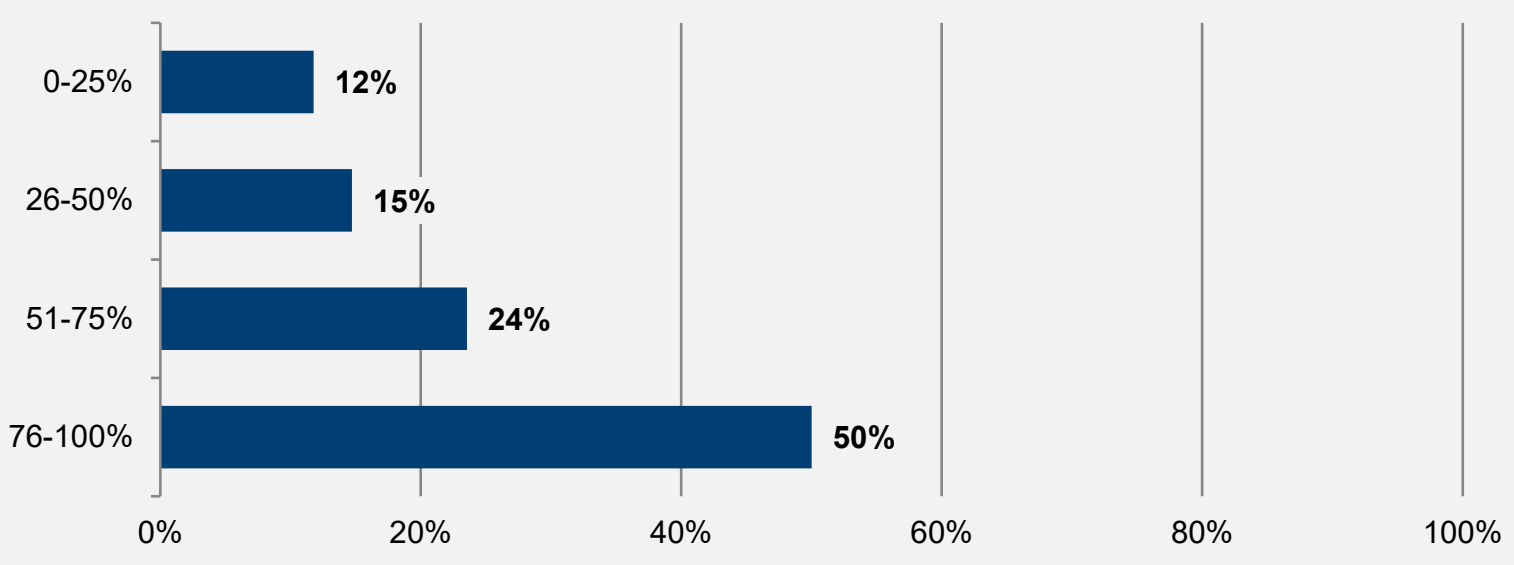

Quelle: ibw-Befragung 2017, n=34

18 Es handelt sich dabei um Tätigkeiten, für deren Ausübung ein hoher Anteil an Kenntnissen in den Bereichen Mathematik, Informatik, Naturwissenschaften und/oder Technik erforderlich ist. Dabei wird auch das Bauen und Instandhalten technischer Anlagen und Geräte als zentraler Bestandteil gezählt. 


\subsection{Erstkontakt und Vorerfahrungen}

Wie bzw. von wem haben Projektbeteiligte erstmals von "Talente regional“ erfahren? Bei den KonsortialpartnerInnen (vgl. Grafik 25) zeigt sich eine relativ breite Streuung der Informationskanäle.

Besonders häufig werden die wissenschaftlichen PartnerInnen aber auch die (vor-)schulischen Bildungseinrichtungen genannt. Danach folgt die offene Kategorie „andere Kontakte/Kanäle“, bei der private Kontakte sowie ArbeitskollegInnen eine wichtige Rolle spielen. Aber auch der Kontakt mit dem BMVIT bzw. der FFG sowie deren Informationen und Veranstaltungen werden als relevant eingestuft. Medienberichte und UnternehmenspartnerInnen spielten hingegen eher eine untergeordnete Rolle.

Bei den wissenschaftlichen PartnerInnen ist außerdem die eigene Recherche mit fast einem Drittel der RespondentInnen besonders stark ausgeprägt.

\section{Grafik 25: Erstkontakt mit „Talente regional“ - Teil 1}

Wie bzw. von wem haben Sie zum ersten Mal von „Talente regional“ bzw. "generation innovation Regionen" erfahren?
- Medienberichte
- Kontakt mit BMVIT/FFG
- Konsortialführung
- Unternehmenspartnerln
- andere Kontakte/Kanäle*

- eigene Recherche

Informationen/Veranstaltungen des BMVIT/FFG

-Wissenschaftliche/r Partnerln

च (vor-)schulische Bildungseinrichtung

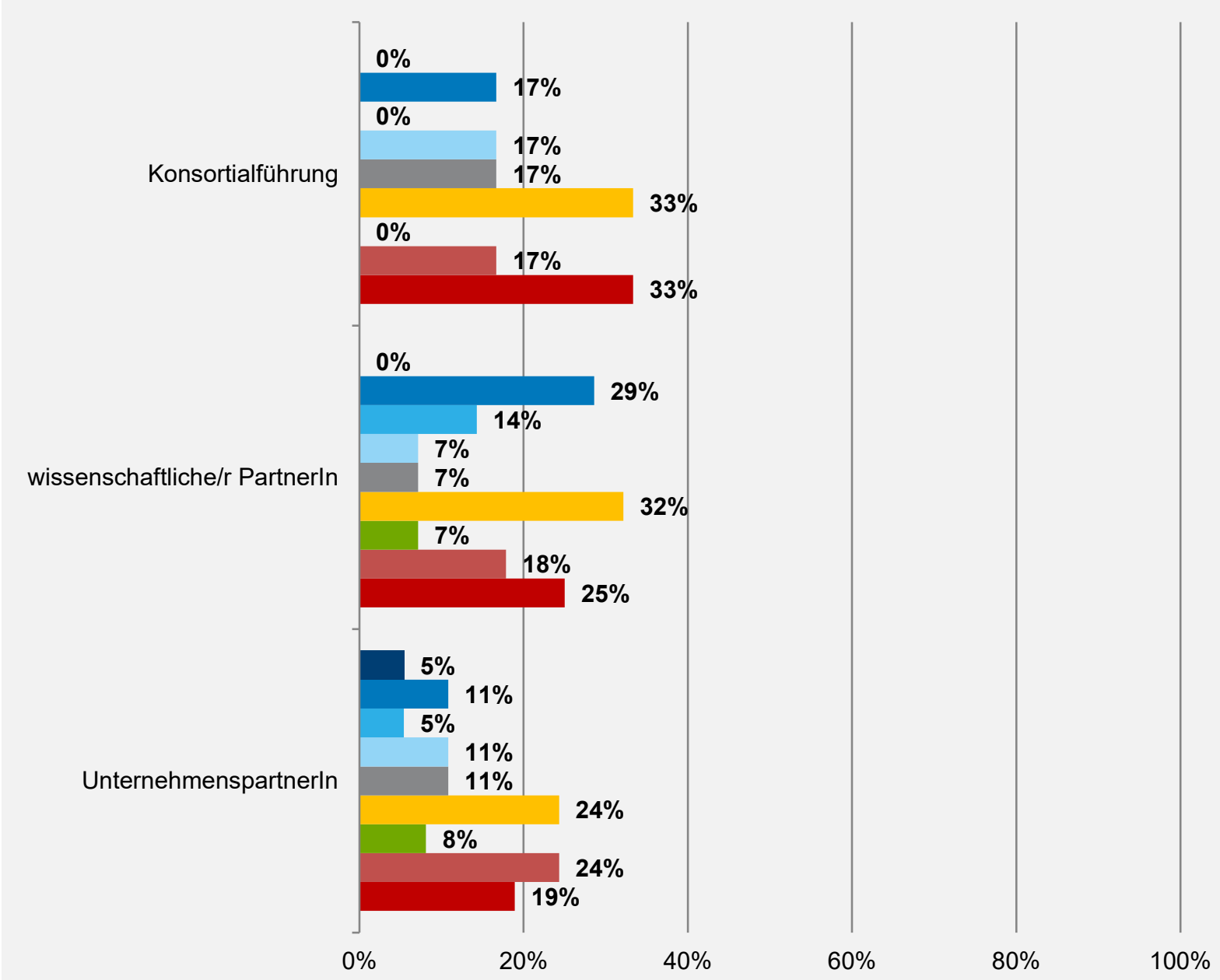

Quelle: ibw-Befragung 2017, n=181

Anmerkung: Unter die Kategorie „andere Kontakte/Kanäle“ fallen private Kontakte sowie Arbeitskolleglnnen. 
Bei den Bildungseinrichtungen dominiert die Konsortialführung als erstmaliger Kontakt mit „Talente regional" (vgl. Grafik 26). Danach folgen mit einem Abstand von 23 bzw. 16 Prozentpunkten die wissenschaftlichen Partnerlnnen. Die Bildungseinrichtungen mit Kooperationszuschuss nennen außerdem die Informationen und Veranstaltungen des BMVIT und der FFG relativ häufig als Anknüpfungspunkt.

Ganz anders sieht es bei den RespondentInnen der Kategorie „andere Rollen/Funktionen“ aus, hier macht die eigene Recherche den höchsten Anteil aus. Danach folgen der Kontakt mit dem BMVIT und der FFG sowie deren Informationen und Veranstaltungen.

\section{Grafik 26: Erstkontakt mit „Talente regional“ - Teil 2}

Wie bzw. von wem haben Sie zum ersten Mal von „Talente regional“ bzw. „generation innovation Regionen“ erfahren?
- Medienberichte
- Kontakt mit BMVIT/FFG
- Konsortialführung
- Unternehmenspartnerln
andere Kontakte/Kanäle*

- eigene Recherche

Informationen/Veranstaltungen des BMVIT/FFG

Wissenschaftliche/r Partnerln

- (vor-)schulische Bildungseinrichtung

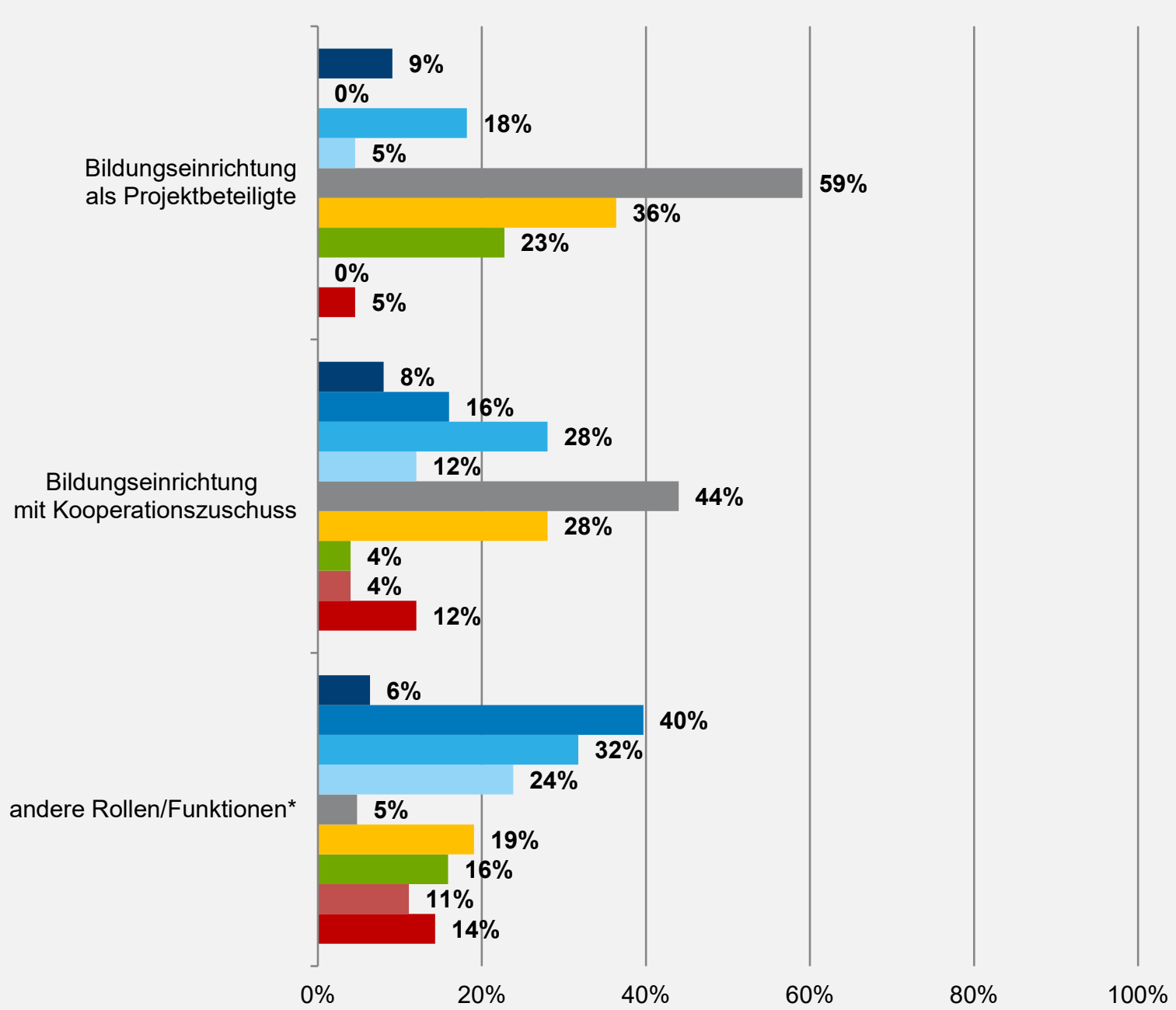

Quelle: ibw-Befragung 2017, $\mathrm{n}=181$

Anmerkung: Unter die Kategorie „andere Kontakte/Kanäle“ fallen private Kontakte sowie ArbeitskollegInnen. Unter die Kategorie „andere Rollen/Funktionen“ fallen sogenannte „assoziierte PartnerInnen“, die im Projekt eine unterstützende Rolle eingenommen, aber keine Kosten geltend gemacht haben. 
Neben den Projektbeteiligungen an „Talente regional“ hat fast ein Drittel der Befragten auch noch an anderen Formaten des Förderschwerpunkts „Talente“ teilgenommen.

Grafik 27: Teilnahme an anderen Förderformaten von „Talente“

Haben Sie im Förderschwerpunkt „Talente“ des BMVIT über Talente regional hinausgehend auch an anderen Förderformaten (bspw. Praktika für Schülerlnnen, FEMtech Karriere, etc.) teilgenommen?

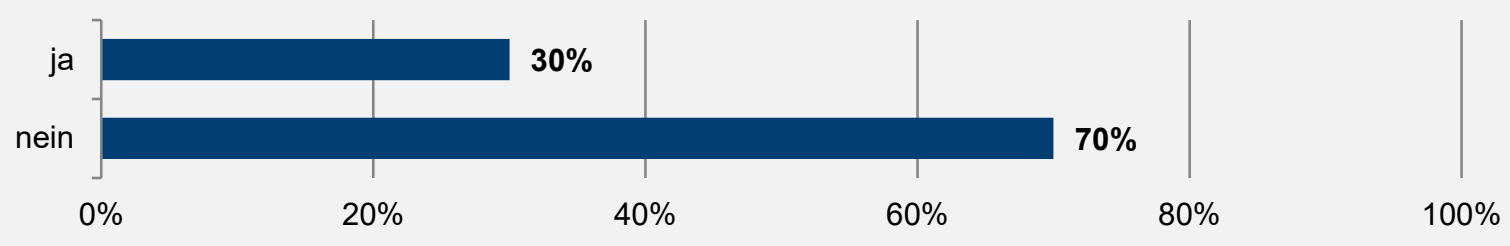

Quelle: ibw-Befragung 2017, n=170

Mit über $80 \%$ entfällt ein Großteil dieser Teilnahmen auf „Praktika für Schülerinnen und Schüler“. Aber auch die „FEMtech Praktika für Studentinnen“ bzw. „FEMtech Forschungsprojekte“ sind mit $41 \%$ bzw. $25 \%$ vertreten. Bei den anderen Formaten dieses Förderschwerpunkts weisen die RespondentInnen verhältnismäßig wenige Teilnahmen auf (vgl. Grafik 28).

\section{Grafik 28: Weitere Förderformate des BMVIT}

\section{Um welches Förderformat handelt es sich genau?}

Praktika für Schülerinnen und Schüler

FEMtech Praktika für Studentinnen

FEMtech Forschungsprojekte

FEMtech Karriere/FEMtech Karriere-Check für $\mathrm{KMU}$

Die österreichische Jobbörse für Forschung, Entwicklung und Innovation.

FEMtech Dissertationen

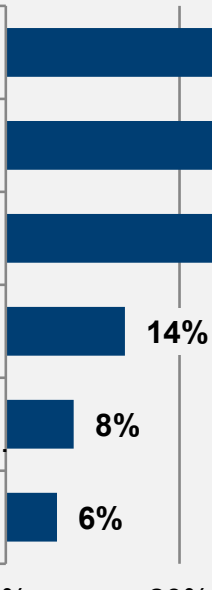

$0 \%$

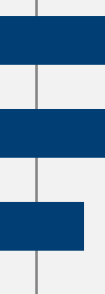

$14 \%$

$25 \%$

$20 \%$

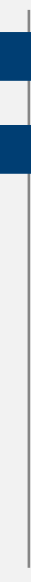

$40 \%$
$82 \%$

$41 \%$

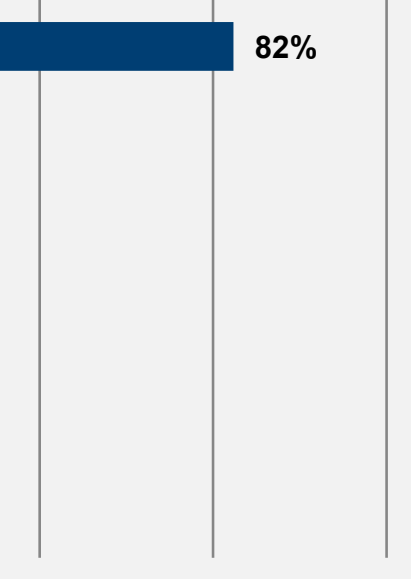

$60 \% \quad 80 \%$

$100 \%$

Quelle: ibw-Befragung 2017, n=51 (Mehrfachnennungen)

Insgesamt 55\% der Befragten geben außerdem an, dass Sie auch schon an anderen Initiativen zur Förderung des Interesses an Naturwissenschaft und Technik teilgenommen haben.

Grafik 29: Teilnahme an anderen Initiativen

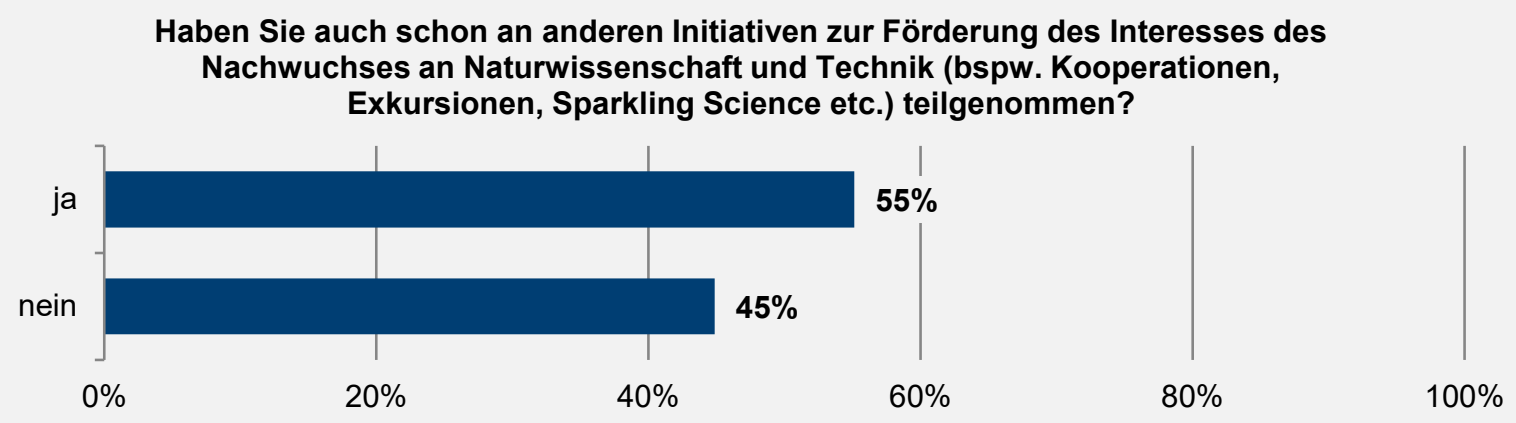

Quelle: ibw-Befragung 2017, n=156 
Davon geben $23 \%$ an, dass sie sich bei „Sparkling Science“ beteiligt hätten. Danach folgen diverse Exkursionen, Besichtigungen, Workshops und Messebesuche mit 20\%. Weitere Initiativen bzw. Projekte wie die „Lange Nacht der Forschung“, "Citizen Science“ oder "Science4girls“ machen jeweils zwischen 3\% und 6\% aus. Der Anteil der verschiedenen Einzelnennungen von Projekten und Aktivitäten wie "Biocosmos" oder die "Campusland-Initiative“ macht zusammen insgesamt $31 \%$ aus (vgl. Grafik 30).

\section{Grafik 30: Initiativen}

Wenn ja, um welche Initiativen handelte es sich?

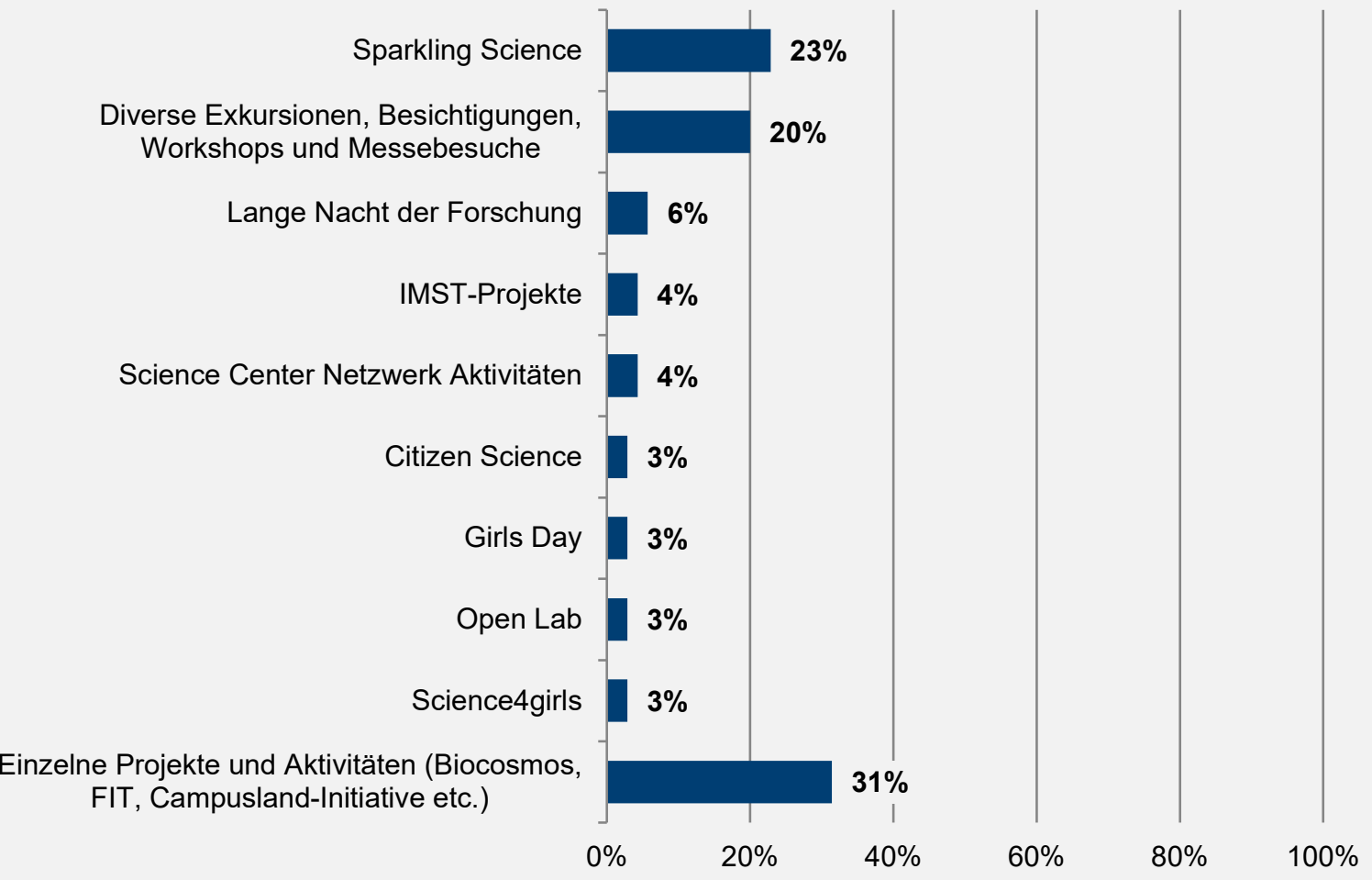

Quelle: ibw-Befragung 2017, n=70 


\subsection{Projektidee und Kontaktaufnahme}

Die in diesem Abschnitt behandelten Fragen wurden ausschließlich an die Konsortialführung gerichtet. Der Großteil, insgesamt $71 \%$, der befragten Konsortialführungen gab an, dass sie selbst den entscheidenden Impuls für das Projekt gegeben haben bzw. die Projektidee hatten. Bei jedem fünften Projekt waren es die UnternehmenspartnerInnen. Die restlichen Organisationen bzw. Kontakte waren jeweils bei jedem zehnten Projekt für den initialen Anstoß verantwortlich (vgl. Grafik 31).

\section{Grafik 31: Projektimpuls}

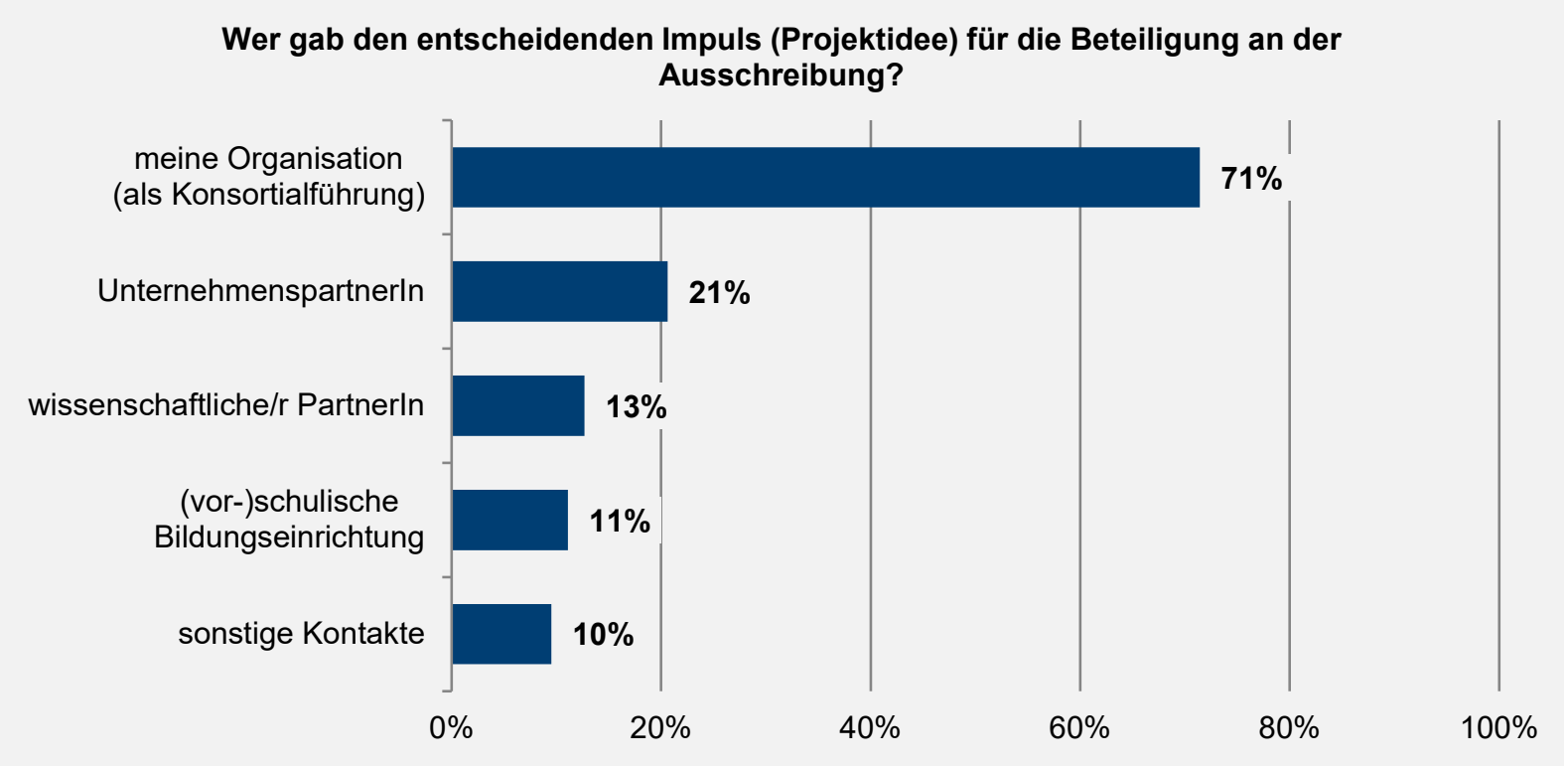

Quelle: ibw-Befragung 2017, n=63 (Mehrfachnennungen möglich)

Anmerkung: Unter die Kategorie „sonstige Kontakte“ fallen private Kontakte sowie ArbeitskollegInnen.

Neben der Projektidee ist auch interessant, wie sich die Kontaktaufnahme zum Projektkonsortium (vgl. Grafik 32) sowie zu den Bildungseinrichtungen (vgl. Grafik 33) gestaltet hat.

Bei den wissenschaftlichen PartnerInnen zeigt sich ein relativ ausgeglichenes Bild. Es handelte sich sowohl um neue Kontakte, als auch um bestehende Netzwerke und Kontakte mit bzw. auch ohne Projektzusammenarbeit in der Vergangenheit. Die kombinierten Kategorien („trifft zu“ und „trifft eher zu“) liegen hier zwischen 58\% und 66\%.

Die Verteilung der Kontaktaufnahme zu den Unternehmenspartnerlnnen ist hingegen etwas stärker durch neue Kontakte geprägt, die erst im Rahmen des Projektes zustande gekommen sind. Hier liegt der kombinierte Anteil („trifft zu“ und "trifft eher zu“) bei genau 70\%. Danach folgen die bestehenden Kontakte und Netzwerke ohne bisherige Projektkooperation mit 59\%. Am geringsten ausgeprägt sind jene, mit denen man in der Vergangenheit bereits zusammengearbeitet hat, mit $47 \%$.

Analog zu den Unternehmenspartnerlnnen ist auch bei den Bildungseinrichtungen die absteigende Häufigkeit der einzelnen Kontaktformen zu beobachten. Bei jenen, die als Projektbeteiligte involviert waren, handelt es sich zu fast $80 \%$ („trifft zu“ und „trifft eher zu“) um gänzlich neue Kontakte. Mit fast $90 \%$ ist dieser Anteil bei den Bildungseinrichtungen, die im Rahmen von Kooperationszuschüssen teilgenommen haben, sogar noch stärker ausgeprägt. 


\section{Grafik 32: Kontaktaufnahme mit dem Konsortium}

\section{Wie kam der Kontakt zum Projektkonsortium zustande?}

Es handelte sich um ...

- trifft zu $\quad$ trifft eher zu $\quad$ trifft eher nicht zu $\quad$ trifft nicht zu

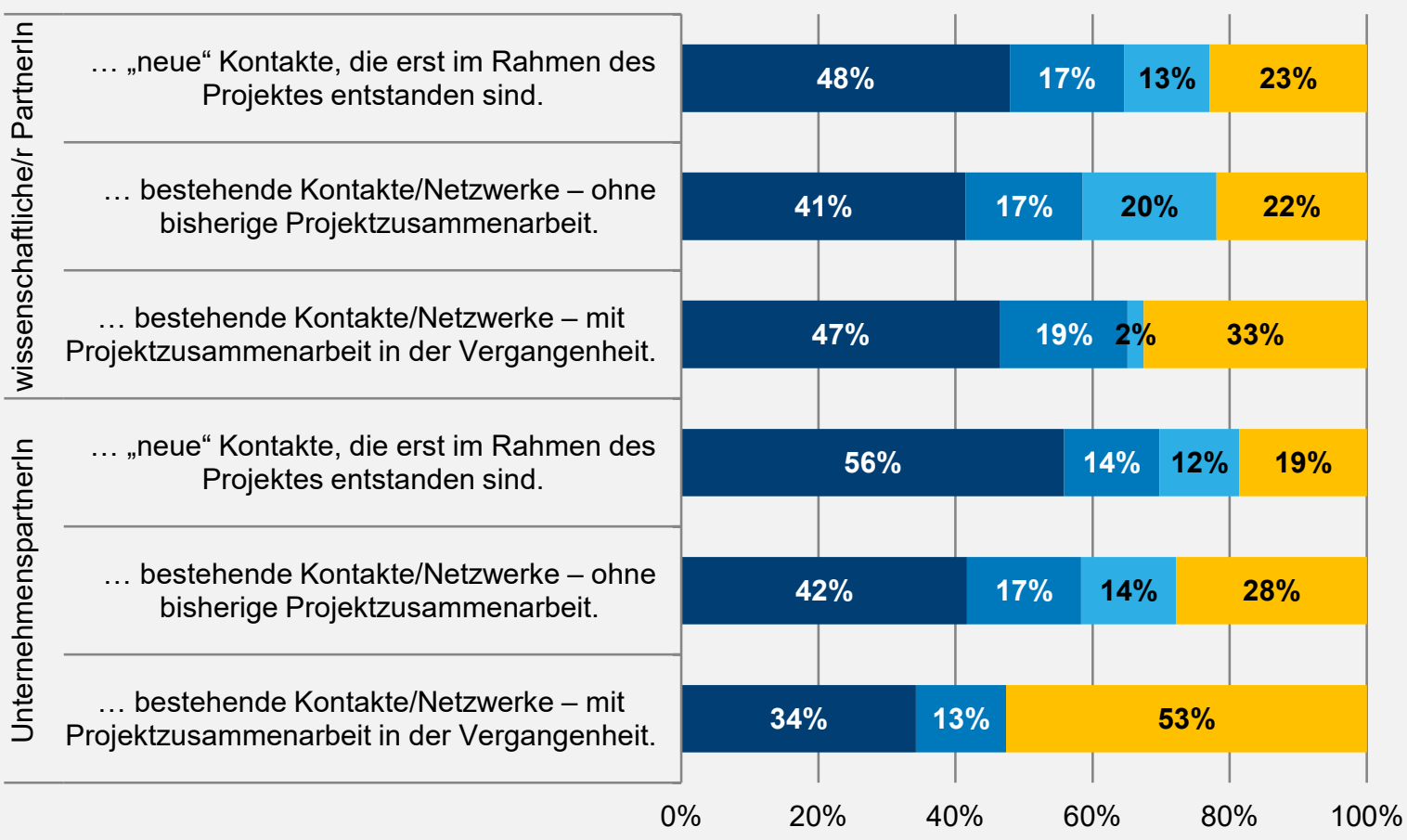

Quelle: ibw-Befragung 2017, n=63

\section{Grafik 33: Kontaktaufnahme mit den Bildungseinrichtungen}

\section{Wie kam der Kontakt zu den (vor-)schulischen Bildungseinrichtungen zustande?} Es handelte sich um ...

- trifft zu $\quad$ atrifft eher zu $\quad$ trifft eher nicht zu $\quad$ trifft nicht zu
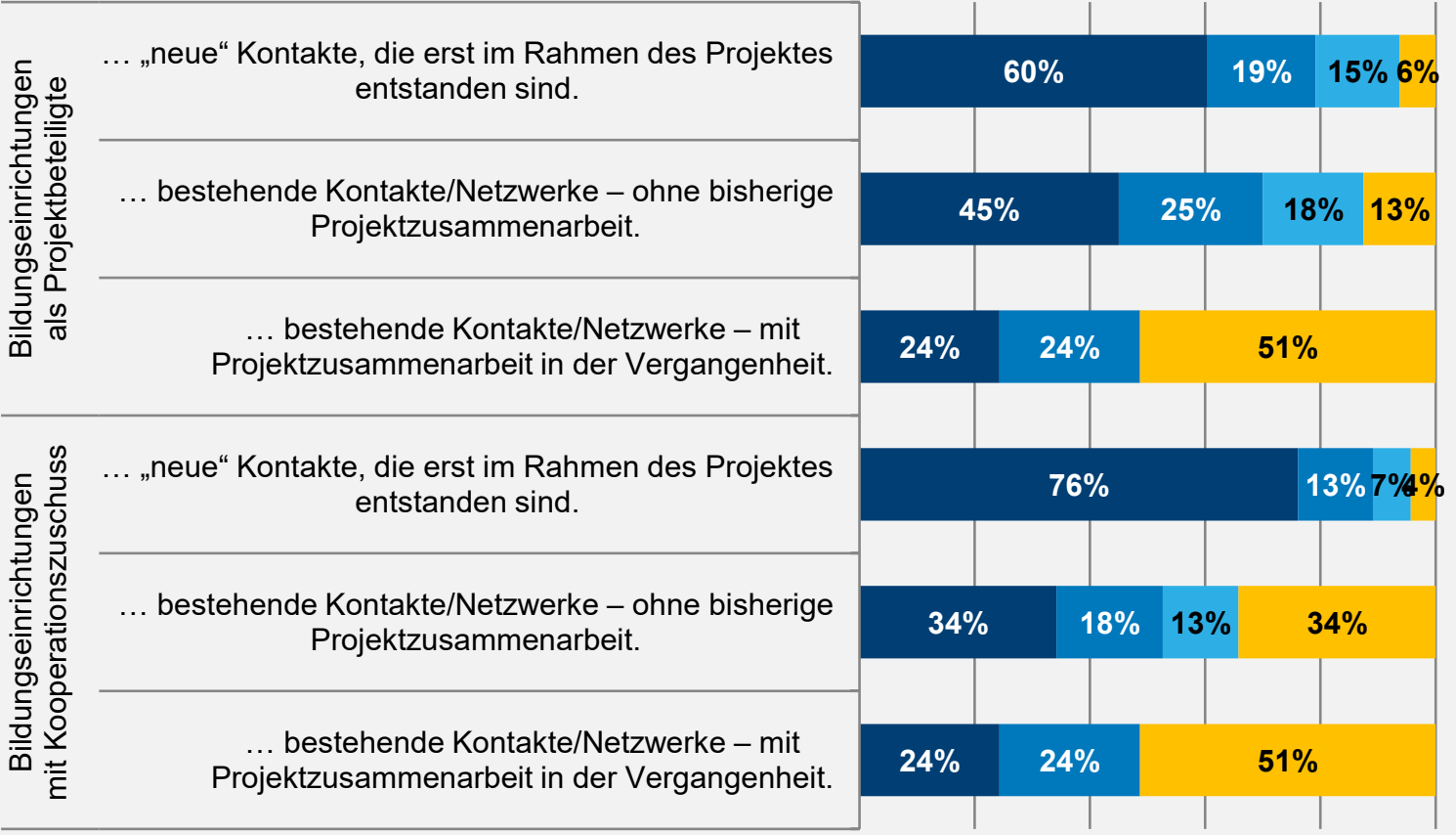


\subsection{Projektabwicklung und -gestaltung}

Die im Abschnitt 4.4 und 4.5 behandelten Fragen wurden an das Projektkonsortium (Konsortialführung, wissenschaftliche PartnerInnen und UnternehmenspartnerInnen) sowie an die Bildungseinrichtungen als Projektbeteiligte gerichtet. Bildungseinrichtungen, die einen Kooperationszuschuss in Anspruch genommen oder andere Rollen und Funktionen eingenommen haben, wurden nicht befragt.

Bei der Frage nach der Zufriedenheit bezüglich verschiedener Aspekte im Zusammenhang mit der Ausschreibung bzw. mit der konkreten Projektabwicklung zeigt sich ein äußerst positives Bild (vgl. Grafik 34). Drei Viertel der RespondentInnen sind mit dem Kontakt sowie der Betreuung durch die FFG sehr zufrieden und ein weiteres Viertel ist zufrieden. Bei den restlichen Items ist die Kategorie „sehr zufrieden“ zwar weniger stark ausgeprägt, liegt allerdings mit mindestens $35 \%$ und maximal $55 \%$ auf einem guten Niveau. Kombiniert mit der Kategorie „zufrieden“ liegt bei allen abgefragten Items der Minimalwert bei $85 \%$. Somit ist es auch wenig überraschend, dass der Höchstwert der Kategorie „nicht zufrieden“ gerade mal bei $5 \%$ liegt. Diese hohen Zufriedenheitswerte legen also nahe, dass der Großteil der RespondentInnen mit der Ausschreibung und Projektabwicklung äußerst zufrieden war.

\section{Grafik 34: Zufriedenheit mit der Ausschreibung und Projektabwicklung}

Wie zufrieden sind Sie mit den verschiedenen Vorgaben der Ausschreibung und der Projektabwicklung?

घehr zufrieden $₫$ zufrieden $\varpi$ weniger zufrieden $₫$ nicht zufrieden

Kontakt bzw. Betreuung durch die FFG

Laufzeit der Projekte

formaler Ablauf der Antragstellung

Zeitpunkt der Ausschreibung

Förderhöhe/Projektbudget

Darstellung der Förderkriterien

Konzeption der Kooperationszuschüsse

Zeitpunkt (Deadline) der Förderentscheidung

Dauer bis zur Förderentscheidung

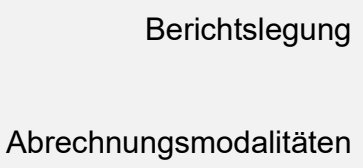

Abrechnungsmodalitäten
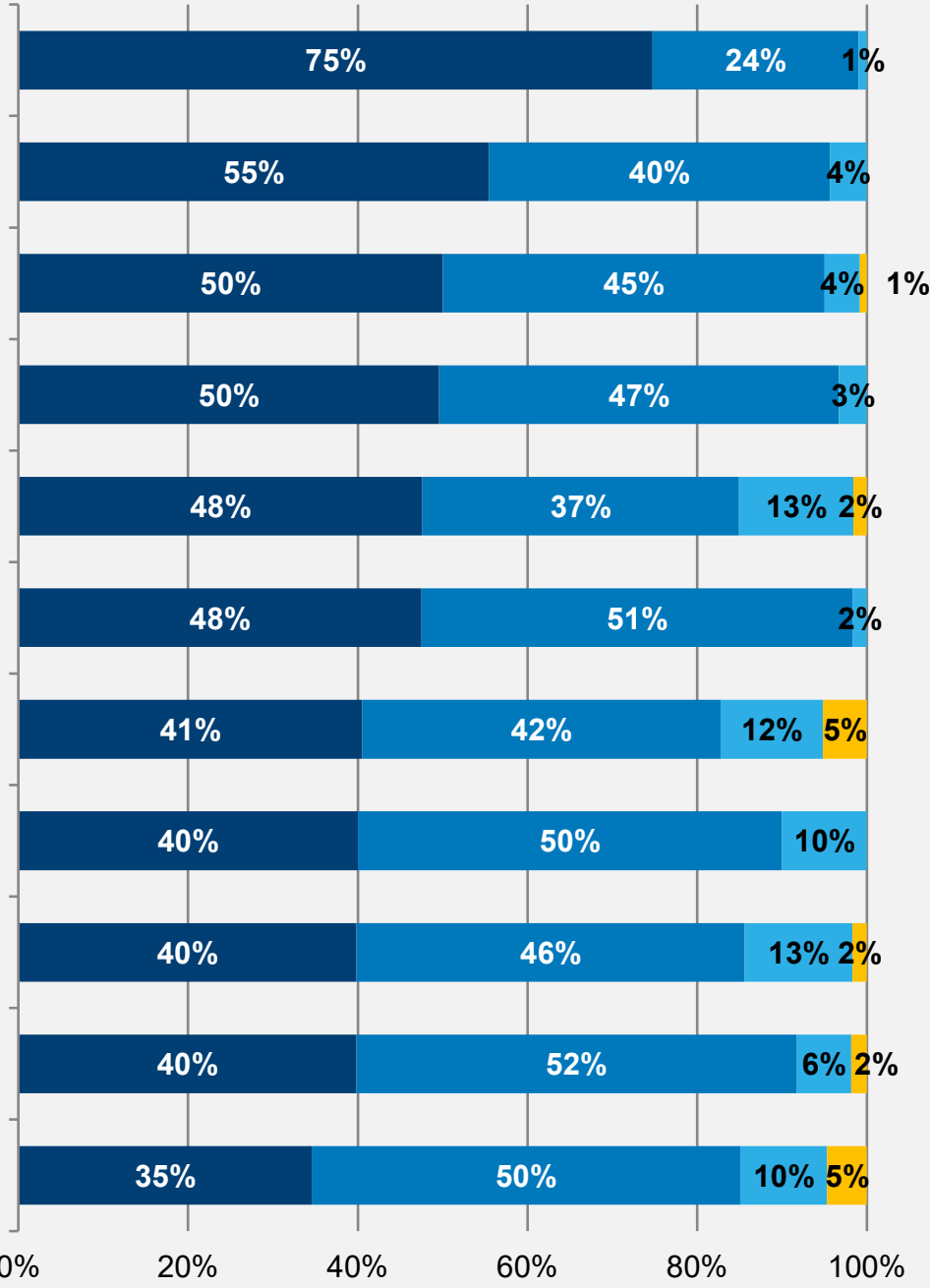
Die Zufriedenheit mit den einzelnen Aspekten innerhalb des Projektkonsortiums wurde sogar noch höher eingestuft als jene mit der Ausschreibung und Projektabwicklung (vgl. Grafik 35). Die Anteile in der Kategorie „sehr zufrieden“ liegen zwischen 57\% und 73\%. Kombiniert mit der Kategoire „zufrieden" reichen die Werte von $93 \%$ bis $100 \%$.

\section{Grafik 35: Zufriedenheit mit dem Projektkonsortium}

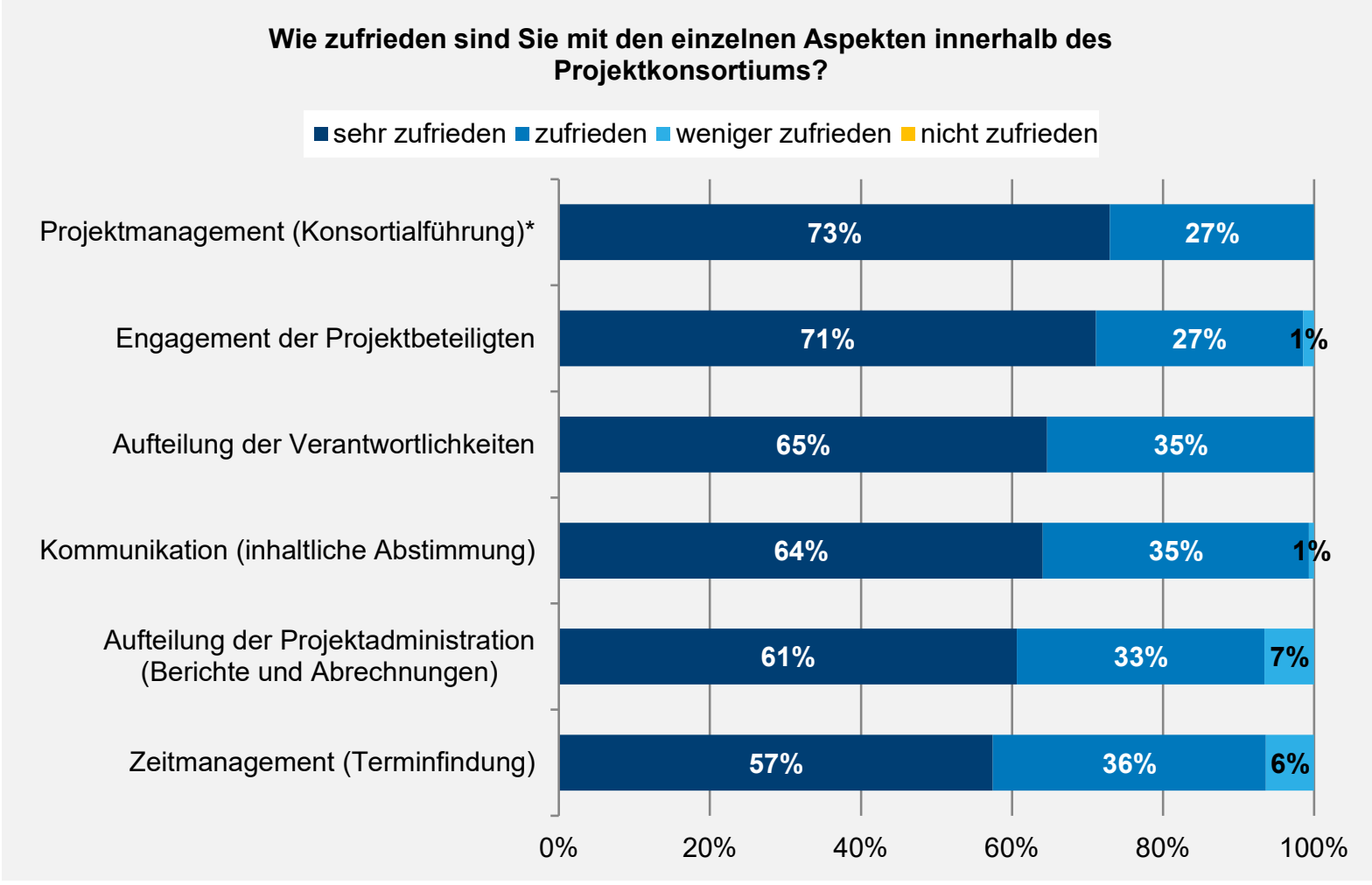

Quelle: ibw-Befragung 2017, n=122-142

Anmerkung: Der Konsortialführung selbst wurde das erste Frage-Item nicht angezeigt, $n=74$ 


\subsection{Ausschreibungsschwerpunkte}

Neben der Zufriedenheit mit den verschiedenen Aspekten der Ausschreibung sowie dem Konsortium wurden die Projektbeteiligten auch noch gefragt, wie sie die Eignung der Ziele und Anforderungen von „Talente regional“ einschätzen.

Bei allen Items (vgl. Grafik 36), abgesehen vom letzten, sind weit über die Hälfte der Respondentlnnen der Meinung, dass "Talente regional“ gut geeignet ist, die genannten Ziele und Anforderungen zu erreichen bzw. umzusetzen. Berücksichtigt man auch noch die Kategorie „trifft eher zu“, so liegen alle Items über $85 \%$, somit wird die Eignung von „Talente regional“ für die Umsetzung der abgefragten Ziele und Anforderungen als überaus hoch eingeschätzt.

Allerdings lässt sich eine Rangreihung ableiten, die zeigt, welche Bereiche sich besonders gut adressieren lassen. So ist "Talente regional“ laut 90\% der RespondentInnen zur Steigerung des Interesses an Forschung, Technologie und Innovation sowie zu $87 \%$ zur Vertiefung des Bezugs der Zielgruppe zu Naturwissenschaft und Technik geeignet. Bei der Stärkung der Zusammenarbeit zwischen den verschiedenen ProjektpartnerInnen und den Bildungseinrichtungen liegt dieser Anteil bei $70 \%$. Die Eignung zur aktiven Einbindung von Kindern und Jugendlichen unabhängig von der sozialen oder geografischen Herkunft trifft laut der RespondentInnen 62\% zu. Am geringsten ist der Wert mit 40\% bei der Berücksichtigung der Gender-Aspekte bzw. Geschlechterausgewogenheit.

\section{Grafik 36: Umsetzung der Ziele und Anforderungen}

Sind Sie der Meinung, dass „Talente regional“ bzw. „generation innovation Regionen" dafür geeignet ist, die folgenden Ziele/Anforderungen (Ausschreibungsschwerpunkte) umzusetzen?

Talente regional ist grundsätzlich dafür geeignet ...

घrifft zu $\quad$ trifft eher zu $\quad$ trifft eher nicht zu $\quad$ trifft nicht zu

... das Interesse von Kinder und Jugendlichen an Forschung, Technologie und Innovation zu steigern.

.. den Bezug zu Naturwissenschaft und Technik von Kindern und Jugendlichen durch die aktive Einbindung in die Projekte zu vertiefen.

... die Vernetzung von (vor-)schulischen Bildungseinrichtungen und PartnerInnen aus Wirtschaft und Forschung zu stärken.

... Kinder und Jugendliche unabhängig ihrer sozialen oder geografischen Herkunft (bspw. mit Migrationshintergrund) aktiv einzubinden.

... Gender-Aspekte bzw. Geschlechterausgewogenheit (bspw. durch gezieltes Ansprechen von Mädchen und jungen Frauen) zu berücksichtigen.

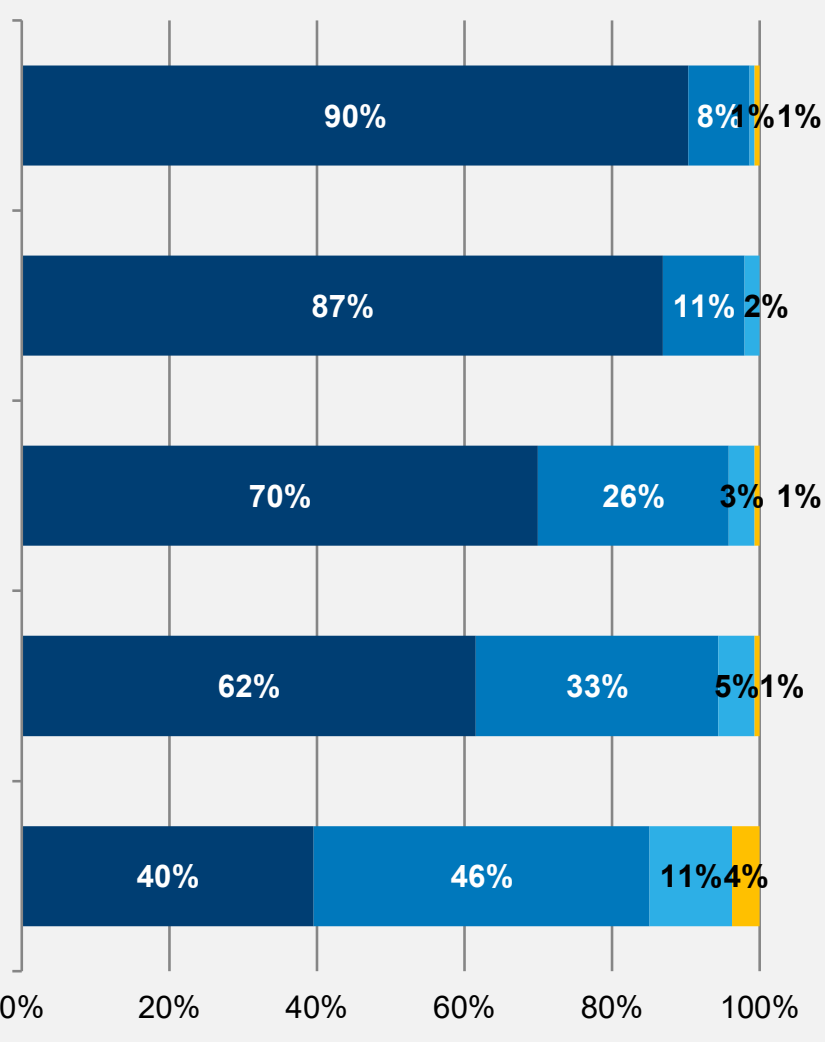


Zusätzlich konnten die RespondentInnen auch noch eine Einschätzung machen, wie anspruchsvoll es war, die genannten Ziele und Anforderungen im Rahmen des jeweiligen Projektes zu berücksichtigen (vgl. Grafik 37). Fast ein Fünftel schätzt dies als sehr anspruchsvoll ein und fast zwei Drittel als eher anspruchsvoll. Lediglich ein Fünftel ist der Meinung, dass dies weniger bzw. gar nicht anspruchsvoll war.

\section{Grafik 37: Anspruch der Ziele und Anforderungen}

Wie anspruchsvoll war es, all diese (oben genannten) Ziele/Anforderungen im Rahmen des Projektes zu berücksichtigen?

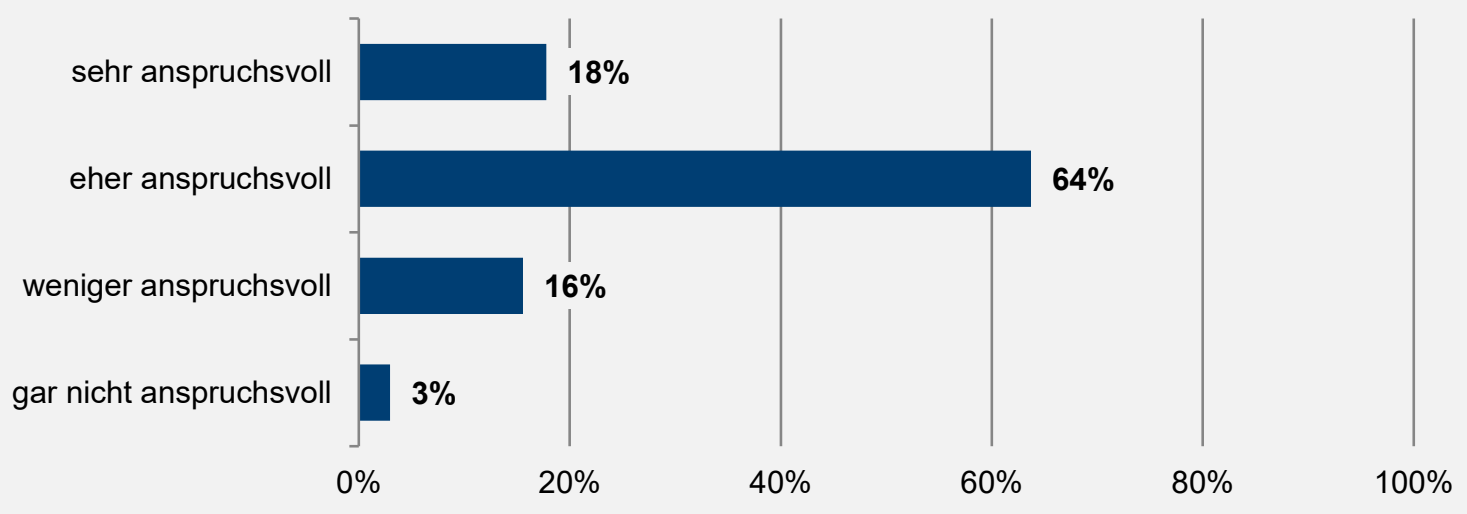

Quelle: ibw-Befragung 2017, n=135-145

Im Rahmen einer offenen Frage gab es auch noch die Möglichkeit anzugeben, wie bzw. in welcher Form die genannten Ziele und Anforderungen im Rahmen des Projektes miteinander in Einklang gebracht werden konnten. Insgesamt haben 71 RespondentInnen eine Angabe gemacht, dies entspricht einem relativen Anteil von 48,3\% (Basis 147 RespondentInnen ${ }^{19}$ ). Die Nennungen lassen sich bezogen auf die jeweilige Abstraktionsebene in die folgenden drei unterschiedlichen Blöcke gruppieren.

Der erste Bereich bezieht sich vor allem auf die Vorbereitung und Tätigkeiten im Rahmen des Projektmanagement. So steht die Koordinierung der Termine und das Zeitmanagement im Rahmen von Steuerungsgruppen und Meetings mit elf Nennungen an erster Stelle. Danach folgen das notwendige Engagement und die hohe Motivation der beteiligten Personen mit neun Nennungen und die intensive Pflege der Kontakte sowie der laufenden Kommunikation mit acht Nennungen. Vorbereitungsmaßnahmen mit den Projektbeteiligten - speziell mit den Schulen bzw. Pädagoglnnen, bspw. im Rahmen von Sensibilisierungsworkshops im Vorfeld - wurden fünf Mal genannt.

Im zweiten Block liegt der Fokus auf der konkreten Projektkonzeption. So geben zehn RespondentInnen an, dass auf gendersensibles Design, also die bewusste Adressierung von Mädchen, gendersensible Sprache und Gestaltung der entwickelten Lehrunterlagen sowie passende Role Models, geachtet werden sollte. Außerdem wird die altersgerechte Umsetzung in Form unterschiedlicher Vermittlungskonzepte, um auch verschiedene Lerntypen zu erreichen, sowie die praxisbezogene Umsetzung mit klarem Bezug zur Anwendbarkeit sechs Mal genannt. Die aktive Einbindung von Schulen mit einem hohen Anteil an SchülerInnen mit Migrationshintergrund hat insgesamt drei Nennungen.

19 Diese Frage wurde an das Projektkonsortium (Konsortialführung, wissenschaftliche Partnerlnnen und UnternehmenspartnerInnen) sowie die Bildungseinrichtungen als Projektbeteiligte gestellt. Bildungseinrichtungen, die einen Kooperationszuschuss in Anspruch genommen haben oder andere Rollen und Funktionen hatten, wurden nicht befragt. 
Beim dritten Bereich steht die methodische Umsetzung der Aktivitäten im Vordergrund. Sieben RespondentInnen geben an, dass vor allem die aktive Einbindung der Kinder/Jugendlichen durch den direkten Dialog und Erfahrungsaustausch bspw. in Form von Teamarbeit mit anschließenden Präsentationen von Bedeutung sind. Aber auch die Anwendung von innovativen Unterrichtsmaterialien und -methoden, bspw. durch Workshops in kleinen Gruppen (damit alle teilnehmenden Kinder und Jugendlichen die Experimente bzw. Übungen auch selbst ausprobieren können) ist wichtig. Exkursionen werden von fünf Befragten als notwendig erachtet, um die Kinder und Jugendlichen in Kontakt mit dem Umfeld von Forschung und Technik (z. B. Betriebe und Werkstätten) zu bringen und die jeweiligen Berufsbilder vor Ort kennen zu lernen. Weiters gibt es auch drei Nennungen, die die unterschiedlichen Coaching-/Mentoring-/Peer-Tutoring-Ansätze als wichtig erachten. 


\subsection{Erfolgsfaktoren}

Die in diesem Abschnitt behandelten Fragen wurden an alle Projektbeteiligten, inklusive Bildungseinrichtungen, die einen Kooperationszuschuss in Anspruch genommen haben, sowie Einrichtungen, die andere Rollen und Funktionen übernommen haben, gestellt.

In der kombinierten Betrachtung der Kategorien „sehr wichtig“ und „wichtig“ wird der Großteil der Items (vgl. Grafik 38 und Grafik 39) als wichtig eingestuft. Abgesehen von den letzten drei Items liegen die Werte zwischen $86 \%$ und $99 \%$ und sind somit überaus hoch. Mit $38 \%$ weist das Item "Einbeziehung der Eltern in die Aktivitäten" den mit Abstand geringsten Wert auf. Danach folgen die "gendersensible Gestaltung der Aktivitäten" mit 57\% und "Coaching/Mentoring/Peer-Tutoring durch ältere SchülerInnen“ mit 64\%.

Betrachtet man ausschließlich die Kategorie „sehr wichtig“, so lässt sich eine deutliche Rangreihung der einzelnen Aspekte identifizieren (vgl. Grafik 38 und Grafik 39). Mit einem relativ großen Abstand wird „eigenes Experimentieren“ mit als $87 \%$ am wichtigsten eingestuft. Danach folgen der persönliche Kontakt zu den ExpertInnen mit $67 \%$, der Besuch der wissenschaftlichen Einrichtungen mit $58 \%$ sowie die Vorbereitung auf die Aktivitäten im Unterricht mit $57 \%$.

Die Hälfte der Befragten stufte auch den Besuch eines innovativen Unternehmens und den Erwerb von technischer Infrastruktur an den Schulen als sehr wichtig ein. Danach folgt ein Bündel von sieben Aspekten, die Werte zwischen $36 \%$ und $45 \%$ aufweisen. Darunter fallen vor allem Aspekte, die sich auf die innerschulische Organisation, die aktive Einbindung der PädagogInnen sowie die didaktische Umsetzung der thematischen Schwerpunkte beziehen.

Analog zur kombinierten Betrachtung der Kategorien weist auch hier das Item „Einbeziehung der Eltern in die Aktivitäten" mit lediglich $9 \%$ den mit Abstand geringsten Wert auf. Danach folgen die "gendersensible Gestaltung der Aktivitäten“ mit 20\% und „Coaching/Mentoring/Peer-Tutoring durch ältere SchülerInnen“ mit 27\%.

\section{Grafik 38: Erfolgsfaktoren - Teil 1}

Welche dieser Aspekte haben sich im Rahmen der Projekte als besonders wichtig erwiesen, um Kinder/Jugendliche für Naturwissenschaft/Technik zu begeistern?

a sehr wichtig

eigenes Experimentieren

persönlicher Kontakt zu ForscherInnen/TechnikerInnen/ Entwicklerlnnen/Wissenschaftlerlnnen

Besuch einer wissenschaftlichen Einrichtung

Vorbereitung auf die Aktivitäten im Unterricht

Besuch eines innovativen Unternehmens

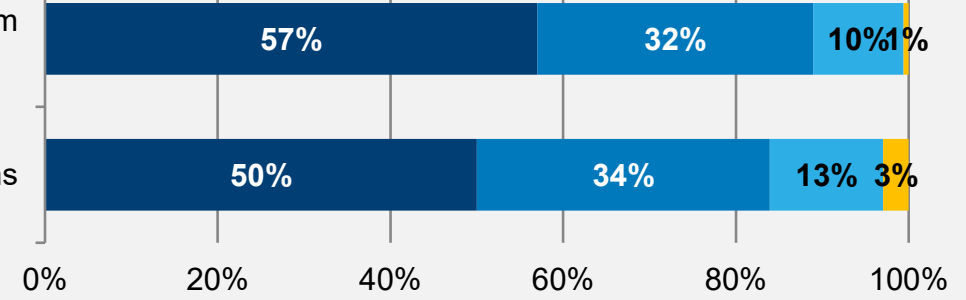

$12 \% 1 \%$
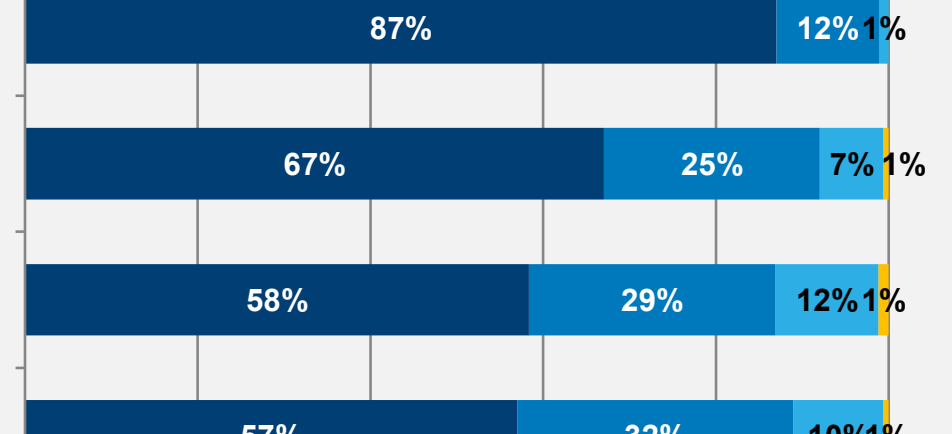

Quelle: ibw-Befragung 2017, n=168-177 


\section{Grafik 39: Erfolgsfaktoren - Teil 2}

Welche dieser Aspekte haben sich im Rahmen der Projekte als besonders wichtig erwiesen, um Kinder/Jugendliche für Naturwissenschaft/Technik zu begeistern?

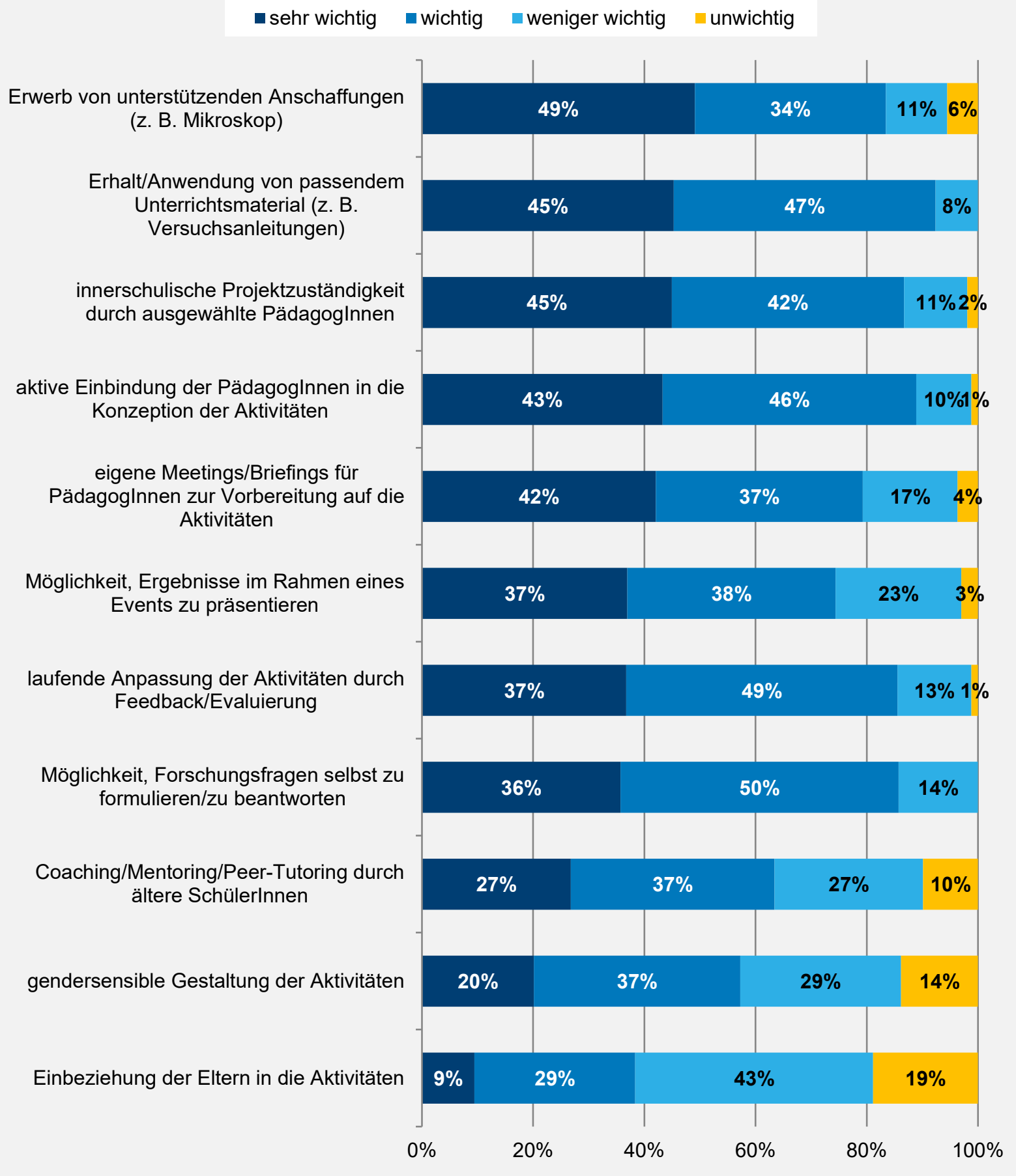

Quelle: ibw-Befragung 2017, n=142-171

Da es sich bei den Projektaktivitäten und den Aktivitäten im Rahmen der Kooperationszuschüsse um verschiedene Maßnahmen handelt, deren Erfolgsfaktoren durchaus unterschiedlich eingeschätzt werden könnten, wurde die Fragestellung zusätzlich differenziert ausgewertet (vgl. Grafik 47 und Grafik 48). Erwähnenswerte Unterschiede ergeben sich bei der Einschätzung bezüglich des Erwerbs von unterstützenden Anschaffungen sowie des Erhalts von passendem Unterrichtsmaterial. Diese beiden Aspekte spielen vor allem im Rahmen der Kooperationszuschüsse eine übergeordnete Rolle und werden aus diesem Grund als sehr wichtig eingestuft. 
Neben der Einstufung der vorgegebenen Items hatten die RespondentInnen im Rahmen einer offenen Frage die Möglichkeit, auch weitere Aspekte zu nennen, die sie aufgrund ihrer Erfahrung als wichtig einschätzen, um Kinder/Jugendliche für Naturwissenschaft/Technik zu begeistern. Insgesamt wurden 51 Angaben gemacht, was einem Anteil von 28\% der Befragten entspricht. Da die verschiedenen Nennungen äußerst vielfältig und heterogen sind, wurde auf eine Gruppierung verzichtet. Der Großteil der folgenden Beschreibungen basiert also auf Einzelnennungen.

So wird darauf hingewiesen, dass die aktive Beteiligung der Kinder und Jugendlichen elementar ist. Damit ist gemeint, dass diese die Möglichkeit bekommen, die Aufgaben wirklich selber auszuprobieren, um die praktischen Arbeiten zu einem gewissen Grad auch eigenständig durchzuführen. Ein weiterer Punkt, der nicht unterschätzt werden sollte, ist die altersgerechte Aufbereitung bzw. Vermittlung der Inhalte sowie die Anknüpfung an die kindliche Lebenswelt, um die Relevanz der Themen für den persönlichen Alltag aufzuzeigen. Um dies zu gewährleisten, wird der Einsatz von kreativen Methoden (bspw. Gamification) und modernen Technologien (bspw. Roboter oder Tablets) genannt. Durch das Filmen der eigenen Aktivitäten können die Maßnahmen gleich vor Ort dokumentiert und auch verbreitet werden. Abschließend wurde auch noch darauf hingewiesen, dass man vermehrt Freiräume (sowohl zeitlich, als auch inhaltlich) im schulischen Altag schaffen müsse, da diese die Grundvoraussetzung für eine erfolgreiche Implementierung von Projekten bzw. Aktivitäten dieser Art sind. 


\subsection{Wirkungen der Projektaktivitäten und Kooperationszuschüsse}

Die in Grafik 40 behandelte Frage wurde an alle Projektbeteiligten, inklusive Bildungseinrichtungen, die einen Kooperationszuschuss in Anspruch genommen haben, sowie Organisationen, die andere Rollen und Funktionen übernommen haben, gestellt.

Bezüglich der Wirkungen bei den Kindern sind etwa zwei Drittel der Befragten der Meinung, dass die ersten drei Wirkungen zutreffen, und ungefähr ein Drittel der Ansicht, dass diese eher zutreffen. Somit ist der Großteil der Meinung, dass bei der Zielgruppe die Kenntnisse erweitert werden konnten, man einen realitätsnahen Einblick in die Tätigkeiten ermöglichen konnte und auch das Interesse an Naturwissenschaft und Technik gestiegen ist. Lediglich die Steigerung des Interesses an den jeweiligen Berufen wird mit 42\% in der Kategorie "trifft zu“ in Relation etwas geringer eingeschätzt.

In der differenzierten Auswertung (vgl. Grafik 49) zeigt sich, dass die Wirkungen der Aktivitäten im Rahmen der Kooperationszuschüsse im Vergleich zu den Projektaktivitäten in allen Items höher eingeschätzt werden. Vor allem die Erweiterung der Kenntnisse in Naturwissenschaft und Technik wird mit $86 \%$ in der Kategorie „trifft zu“ überaus hoch eingeschätzt.

\section{Grafik 40: Wirkungen bei den Kindern und Jugendlichen}

Welche Wirkungen hatten Ihrer Meinung nach die im Projekt durchgeführten Aktivitäten bei den Kindern/Jugendlichen?

- trifft zu $\quad$ trifft eher zu $\quad$ trifft eher nicht zu $\quad$ trifft nicht zu

Die Kenntnisse in Naturwissenschaft und Technik konnten erweitert werden.

Die Projekte ermöglichen einen realitätsnahen Einblick in die Tätigkeit von TechnikerInnen/ForscherInnen.

Das Interesse an Naturwissenschaft und Technik ist gestiegen.

Das Interesse an Berufen in Naturwissenschaft und Technik ist gestiegen.

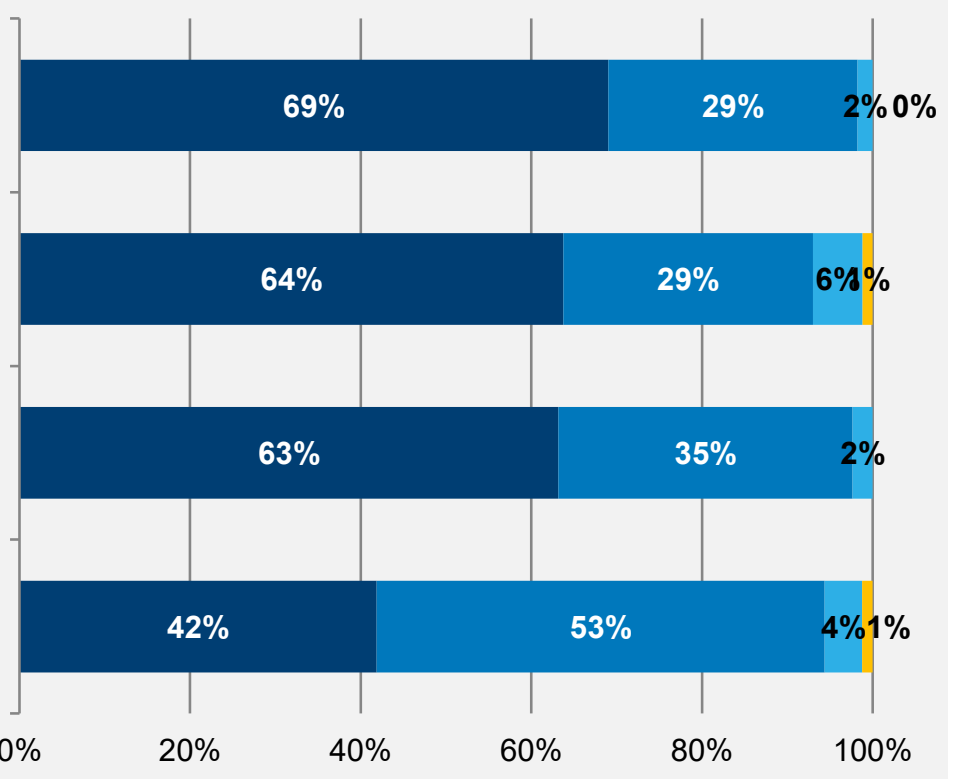

Quelle: ibw-Befragung 2017, n=160-171

Die in Grafik 41 behandelte Frage wurde ausschließlich an Bildungseinrichtungen (inklusive jene mit Kooperationszuschuss) gestellt. Im Vergleich zu den Wirkungen bei Kindern und Jugendlichen ergeben sich bei der Betrachtung der PädagogInnen schon etwas größere Differenzen zwischen den abgefragten Items. Zwar ist die kombinierte Kategorie („trifft zu“ und „trifft eher zu“) bei den ersten drei Items mit Werten zwischen $87 \%$ und $94 \%$ ähnlich hoch, aber anhand der Kategorie "trifft zu“ lässt sich eine Rangordnung ableiten. Am höchsten wird das Kennenlernen von Methoden mit $59 \%$ und das erhöhte Interesse, künftig mehr Projektarbeit in den Unterricht zu integrieren, mit 55\% eingeschätzt. 
Der realitätsnahe Einblick in die Unternehmen im Bereich Naturwissenschaft und Technik wird laut $41 \%$ ermöglicht. Lediglich das Kennenlernen von gendersensibler Unterrichtsgestaltung wird mit $17 \%$ in der Kategorie "trifft zu“ als eher unwahrscheinlich eingeschätzt.

Da es sich bei Projektaktivitäten und den Aktivitäten im Rahmen der Kooperationszuschüsse um verschiedene Maßnahmen handelt, deren Wirkungen durchaus unterschiedlich eingeschätzt werden könnten, wurde die Fragestellung zusätzlich differenziert ausgewertet (vgl. Grafik 50). Die Wirkungen der Aktivitäten im Rahmen der Kooperationszuschüsse werden im Vergleich zu den Projektaktivitäten in allen Items höher eingeschätzt. Bei der Reihung der einzelnen Items ergeben sich allerdings keine Verschiebungen.

\section{Grafik 41: Wirkungen bei den PädagogInnen}

Welche Wirkungen hatten Ihrer Meinung nach die im Projekt durchgeführten Aktivitäten bei den PädagogInnen?

- trifft zu $\quad$ trifft eher zu $\quad$ trifft eher nicht zu $\quad$ trifft nicht zu

Es wurden Methoden kennengelernt, um das Interesse an Naturwissenschaft und Technik bei Kindern/Jugendlichen zu erhöhen.

Die Projekte erhöhten das Interesse künftig mehr Projektarbeit in den Unterricht zu integrieren.

Die Projekte ermöglichen den PädagogInnen einen realitätsnahen Einblick in Unternehmen im Bereich der Naturwissenschaft und Technik.

Es wurden Methoden zur gendersensiblen Unterrichtsgestaltung kennengelernt.

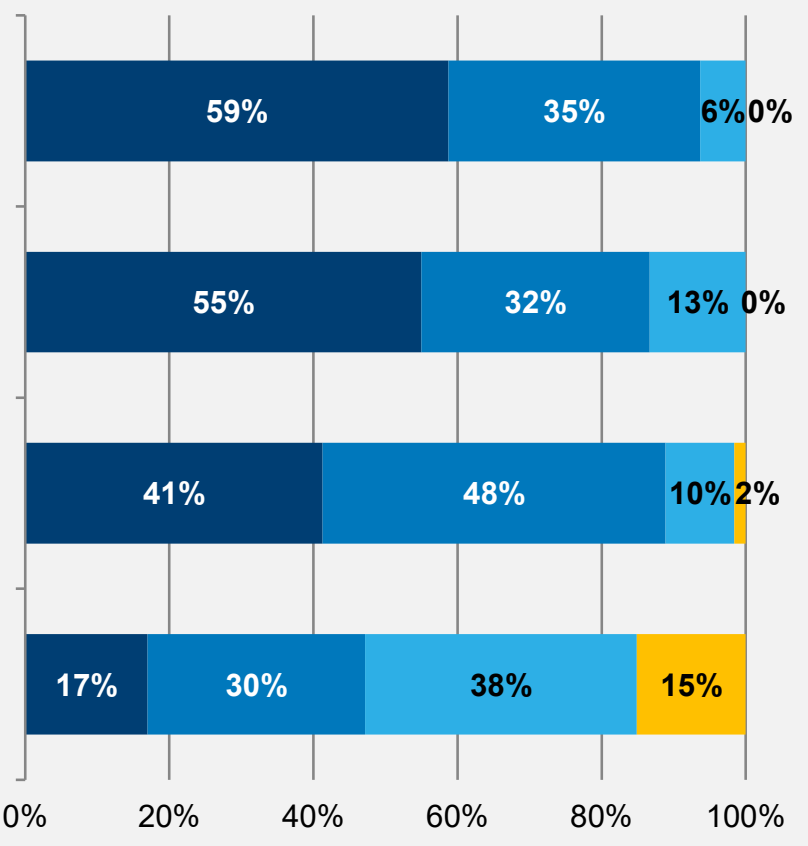

Quelle: ibw-Befragung 2017, n=53-63

Die in Grafik 42 behandelte Frage nach den Wirkungen auf Ebene der Schulen wurde ausschließlich an Bildungseinrichtungen (inklusive jene mit Kooperationszuschuss) gestellt. Im Vergleich zu den Wirkungen bei den Kindern und Jugendlichen sowie den PädagogInnen werden jene auf der Ebene der Schulen etwas geringer eingestuft. Bei der Betrachtung der Kategorie „trifft zu“ ergeben sich relativ geringe Unterschiede zwischen den einzelnen Items. Die Werte liegen lediglich zwischen $22 \%$ und $30 \%$.

Zieht man die Werte der kombinierten Kategorie heran („trifft zu“ und „trifft eher zu“), so ergibt sich eine Rangreihung der einzelnen Items. Vor allem die Steigerung des Stellenwerts von Naturwissenschaft und Technik im Unterricht ist mit einem Wert von $88 \%$ relativ hoch. Danach folgt die Stärkung der schulinternen Zusammenarbeit mit $78 \%$. Schwächer ausgeprägt sind die Verbesserung der technischen Infrastruktur an der Schule mit 56\% und der Aufbau von regionalen Kooperationen mit Wirtschaft und Forschung mit 49\%. 
Die Projektaktivitäten und die Aktivitäten im Rahmen der Kooperationszuschüsse wurden getrennt ausgewertet (vgl. Grafik 51) und zeigen Folgendes. Analog zu den Wirkungen bei den Kindern und Jugendlichen sowie PädagogInnen werden auch jene auf der Ebene der Schulen von den Bildungseinrichtungen mit Kooperationszuschuss etwas höher eingeschätzt. Insbesondere die Verbesserung der technischen Infrastruktur sticht mit $44 \%$ in der Kategorie "trifft zu“ und $40 \%$ in der Kategorie "trifft eher zu" stark heraus. Im Vergleich liegen die Anteile bei den Bildungseinrichtungen als Projektbeteiligte lediglich bei $6 \%$ bzw. $30 \%$. Dies ist allerdings nicht überraschend, da mit den Kooperationszuschüssen die Bildungseinrichtungen die Möglichkeit erhalten, zusätzlich innovative Unterrichtsprojekte im Bereich Naturwissenschaft und Technik an Kindergärten und Schulen bei deren Umsetzung zu fördern.

\section{Grafik 42: Wirkungen auf der Ebene der Schulen}

Welche Wirkungen hatten Ihrer Meinung nach die im Projekt durchgeführten Aktivitäten auf der Ebene der Schulen?

Quelle: ibw-Befragung 2017, n=55-59

Die in Grafik 43 behandelte Frage nach den Wirkungen der Kooperationszuschüsse wurde an alle Projektbeteiligte, inklusive Bildungseinrichtungen, die einen Kooperationszuschuss in Anspruch genommen haben, sowie Organisationen, die andere Rollen und Funktionen übernommen haben, gestellt. Nicht befragt wurden jene RespondentInnen, welche ausschließlich eine Projektbeteiligung im Jahr 2010 aufweisen, da in dieser Ausschreibung (generation innovation Regionen) noch keine Kooperationszuschüsse vergeben wurden.

Betrachtet man die kombinierten Kategorien („trifft zu“ und "trifft eher zu“), so ergeben sich nur geringe Unterschiede. Da keines der Items einen geringeren Wert als $80 \%$ aufweist, treffen laut den Befragten offenbar alle der genannten Wirkungen zu einem relativen hohen Grad zu.

Auch auf Basis der Kategorie „trifft zu“ sind die Unterschiede eher schwach. So findet die Einschätzung, dass die Kooperationszuschüsse eine Förderung mit einem geringen formalen Aufwand ermöglicht, mit $60 \%$ die meiste Zustimmung. Danach folgen die Vergrößerung der angesprochenen Zielgruppe mit $56 \%$, die Erweiterung der Vielfalt der ProjektteilnehmerInnen mit $53 \%$ und die Basis für eine breitere Vernetzung zwischen Schule, Wirtschaft und Forschung mit 50\%. Die Förderung des 
Interesses, sich vermehrt mit Förderungen auseinanderzusetzen trifft laut $45 \%$ zu. An letzter Stelle liegt der Beitrag, die Projekte von „Talente regional“ in der Öffentlichkeit bekannter zu machen, mit $36 \%$.

\section{Grafik 43: Wirkungen der Kooperationszuschüsse}

\section{Welche Wirkungen haben Ihrer Meinung nach die vergebenen Kooperationszuschüsse?}

- trifft zu $\quad$ atrifft eher zu $\quad$ trifft eher nicht zu $\quad$ trifft nicht zu

Sie ermöglichen die Förderung von Aktivitäten mit einem geringen formalen Aufwand.

Sie vergrößern die Anzahl der angesprochenen Kinder und Jugendlichen im hohen Maße.

Sie erweitern die Vielfalt der Projektteilnehmerlnnen entscheidend.

Sie sind eine gute Basis für die breitere Vernetzung von (vor-)schulischen Bildungseinrichtungen mit PartnerInnen aus Wirtschaft und Forschung.

Sie begünstigen das Interesse, sich vermehrt mit der Inanspruchnahme von Förderungen auseinanderzusetzen.

Sie tragen wesentlich zur Bekanntheit von „Talente regional" und deren Projekten in der Öffentlichkeit bei.

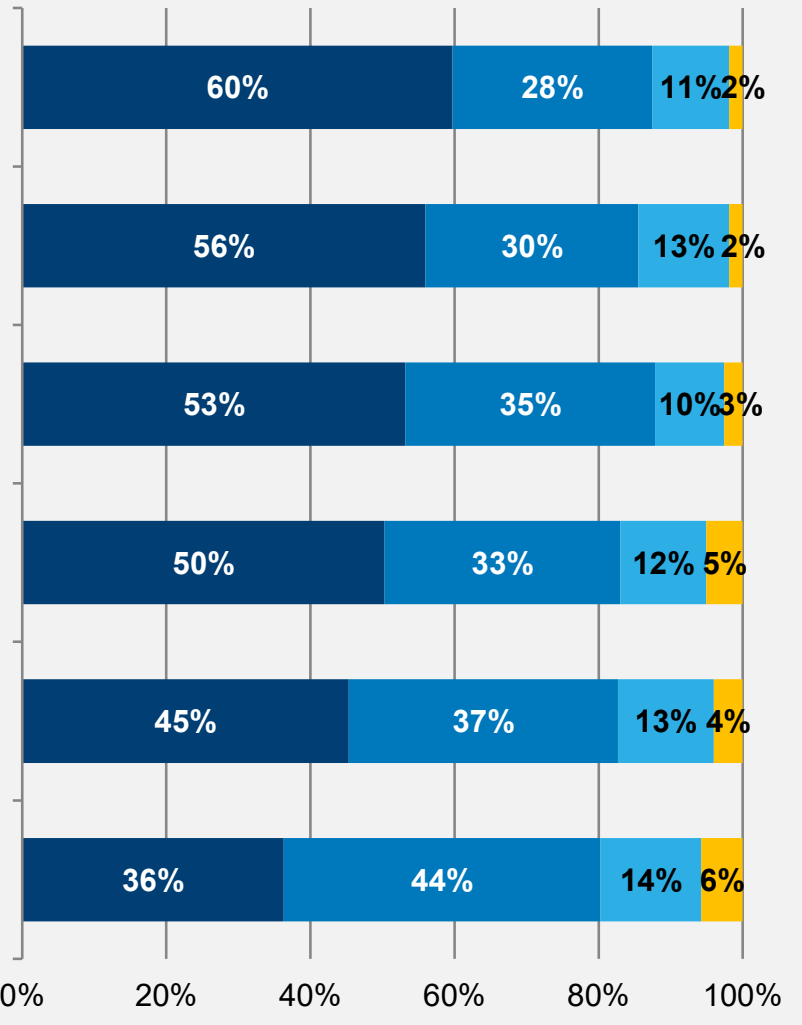

Quelle: ibw-Befragung 2017, n=150-159 


\subsection{Nachhaltigkeit der Kooperationen}

Die in diesem Abschnitt behandelten Fragen wurden an alle Projektbeteiligten, inklusive Bildungseinrichtungen, die einen Kooperationszuschuss in Anspruch genommen haben, sowie Organisationen, die andere Rollen und Funktionen übernommen haben, gestellt. Da die Projekte aus den Ausschreibungen aus dem Jahr 2015 und 2016 zur Zeit der Befragung erst seit kurzem bzw. noch nicht abgeschlossen waren, wurden jene RespondentInnen, die ihre Antworten auf eine Projektbeteiligung dieser Ausschreibungen beziehen, nicht befragt. Von der ausgewählten Stichprobe gaben fast drei Viertel der Befragten an, dass nach Projektabschluss nachhaltige Kooperationen mit einzelnen Organisationen entstanden seien.

\section{Grafik 44: Kooperationen nach Projektabschluss}

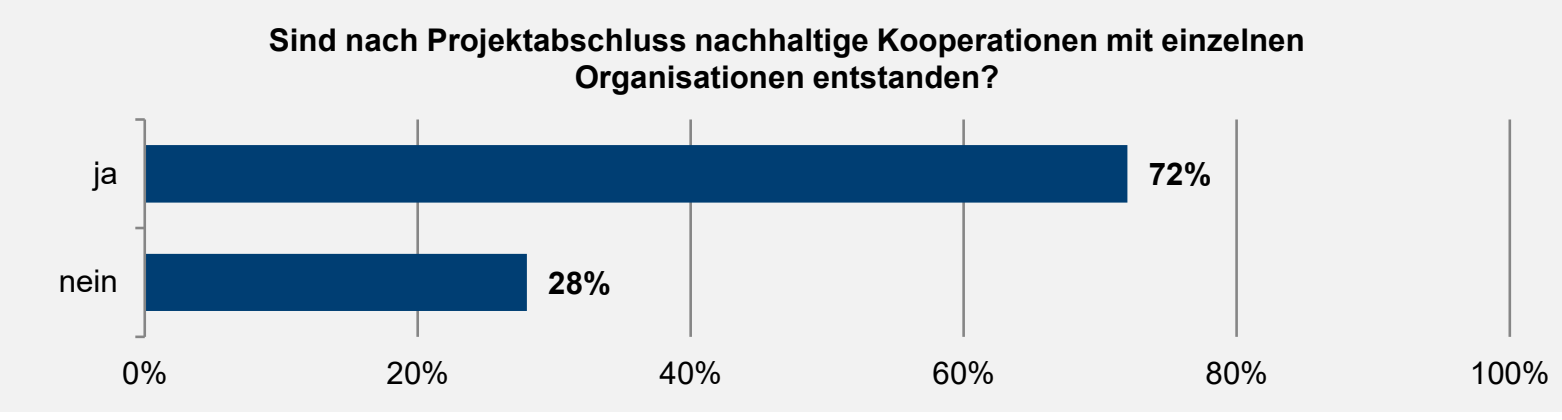

Quelle: ibw-Befragung 2017, n=50

Kooperationen mit einer starken Zusammenarbeit finden vor allem bei den (vor-)schulischen Bildungseinrichtungen (41\%) und Intermediären (32\%) statt. Auf Kooperationen mit Unternehmen, Hochschulen und außeruniversitären Forschungseinrichtungen trifft dies bei einem Viertel bzw. Fünftel der Befragten zu (vgl. Grafik 45).

Interessant ist, dass die Anteile der Zusammenarbeit bei mittelmäßiger Intensität vor allem bei den Hochschulen, Unternehmen und außeruniversitären Forschungsreinrichtungen, mit $40 \%$ bis $52 \%$, besonders hoch sind.

Grafik 45: Intensität der Zusammenarbeit

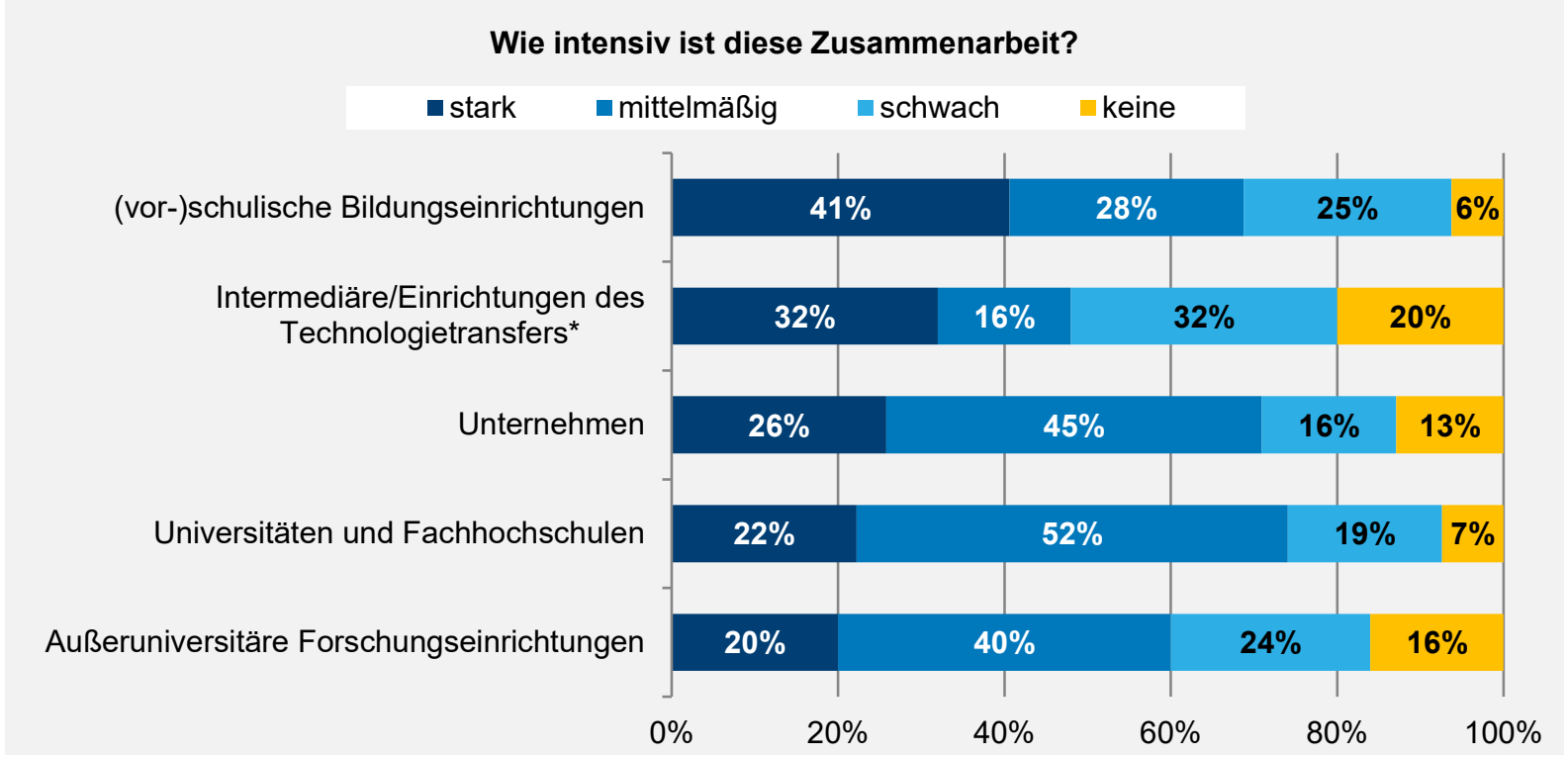

Quelle: ibw-Befragung 2017, n=25-32

Anmerkung: Unter die Kategorie „Intermediäre/Einrichtungen des Technologietransfers“ fallen Gründungs- und Technologiezentren, Unternehmenscluster, Science Center und Regionalmanagements. 
Bei der Betrachtung der kombinierten Kategorie („trifft zu“ und „trifft eher zu“) zeigt sich, dass die Formen der Zusammenarbeit relativ vielfältig sind (vgl. Grafik 46). Einerseits wird zwar indirekt an die Arbeiten des abgeschlossenen Projektes angeknüpft (78\%) und andererseits das pädagogische bzw. didaktische Konzept weiterentwickelt $(72 \%)$. Aber auch andere thematische Schwerpunkte sowie Zielgruppen gibt es laut $68 \%$ bzw. $54 \%$ der befragten Stichprobe.

\section{Grafik 46: Formen der Zusammenarbeit}

Um welche Form/en der Zusammenarbeit handelt es sich?

Die Zusammenarbeit ...

- trifft zu $\quad$ trifft eher zu $\quad$ trifft eher nicht zu $\quad$ trifft nicht zu

... entwickelt das pädagogisch/didaktische Konzept weiter.

... schließt direkt an das Projekt an

... hat einen anderen thematischen Fokus.

... hat eine andere Zielgruppe.

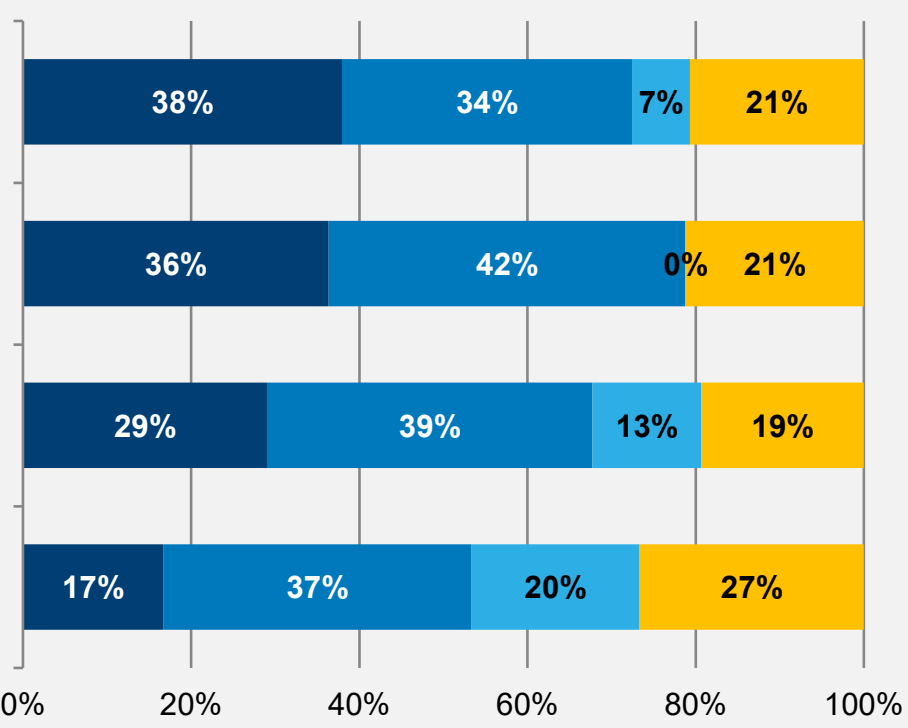

Quelle: ibw-Befragung 2017, n=29-33

Jene Befragten, bei denen es zu keinen nachhaltigen Kooperationen nach Projektabschluss gekommen ist, wurden im Rahmen einer offenen Frage zu den jeweiligen Gründen befragt.

Insgesamt haben 5 RespondentInnen diese Möglichkeit genutzt und folgende Gründe angeführt:

- abgelehntes Förderansuchen

- anderer inhaltlicher Schwerpunkt

- zu hoher Aufwand

- $\quad$ kein wirtschaftlicher Anreiz

- Lehrplan ist zu umfassend und dicht 


\subsection{Offene Abschlussfragen}

Abschließend wurden alle Befragten nochmals gebeten, ihre Einschätzungen und Erfahrungen, die sie bei der Umsetzung des Projekts gemacht haben, im Rahmen von offenen Antworten mitzuteilen. Weiters wurden Projektbeteiligte, die bereits in mehrere Projekte involviert waren, nach erwähnenswerten Unterschieden befragt.

Die erste Frage fokussiert auf die Herausforderungen, mit denen man im Laufe des Projekts konfrontiert war. Hier haben insgesamt 121 RespondentInnen (67\% der Befragten) eine Angabe gemacht. Inhaltlich lassen sich diese Angaben grob in allgemeine Anmerkungen zur Durchführung und Organisation der Projekte sowie Anregungen zur konkreten Umsetzung der Maßnahmen einteilen.

\section{Herausforderungen im Rahmen der Organisation}

- Koordination/Abstimmung/Terminfindung mit den Projektbeteiligten (46 Nennungen, 25\% der Befragten)

- Zeitaufwand/-management vor allem mit den Bildungseinrichtungen sowie kurze Projektlaufzeit (22 Nennungen, 12\% der Befragten)

- Finden von Kooperationsschulen und Ansprechpersonen sowohl als Projektbeteiligte als auch bei Kooperationszuschüssen (9 Nennungen, 5\% der Befragten)

- administrative Vorgaben der Antragstellung und Personalverrechnung sowie zu große Abstände zwischen den Zeitpunkten der Auszahlungen (8 Nennungen, 4\% der Befragten)

- wechselnde Kontaktpersonen (4 Nennungen, $2 \%$ der Befragten)

- zu geringes Projektbudget (4 Nennungen, $2 \%$ der Befragten)

- zu hoher Kommunikationsaufwand (2 Nennungen, 1\% der Befragten)

\section{Herausforderungen im Rahmen der Umsetzung}

- Technik kindgerecht, einfach und spannend erklären - Übersetzung technischer und naturwissenschaftlicher Inhalte in einfache Sprache - Hineindenken in die Aufnahmefähigkeit und Interessenlage von Jugendlichen sowie Berücksichtigung des Wissensstands (15 Nennungen, 8\% der Befragten)

- zusätzliche Themen in den ohnehin engen Lehrplan integrieren (6 Nennungen, $3 \%$ der Befragten)

- Überzeugungsarbeit im Kollegium sowie Motivation der PädagogInnen (5 Nennungen, 3\% der Befragten)

- Ausstattung der einzelnen Forschergruppen - Technischer Fortschritt: teilweise sind Anschaffungen schnell wieder veraltet (3 Nennungen, 2\% der Befragten)

- Aufmerksamkeit der SchülerInnen eine längere Zeit aufrecht zu halten (2 Nennungen, 1\% der Befragten) 
Bei der zweiten Frage wurde nach konkreten Vorschlägen für Verbesserungen gefragt. Hier haben insgesamt 68 RespondentInnen (38\% der Befragten) eine Angabe gemacht.

- Bildungseinrichtungen (17 Nennungen, 9\% der Befragten)

Informationskampagnen, Online-Pool von interessierten Bildungseinrichtungen, Anreize für Bildungseinrichtungen schaffen, Bildungseinrichtungen früher einbinden bspw. durch Vorbesprechungen und Weiterbildungen, Lehrplan entrümpeln, weniger/homogenere Altersgruppen

- Konzeption der Projekte (6 Nennungen, 3\% der Befragten) breitere Themenbereiche, weniger Innovationsgehalt dafür mehr Tiefe und Folgeprojekte, stärkere Trennung der Schwerpunkte

- Kooperationszuschüsse (6 Nennungen, $3 \%$ der Befragten) zentraler Pool, Information durch Ministerium, geringeres Stundenausmaß, eigene Förderschiene

- Projektbudget (5 Nennungen, 3\% der Befragten)

höherer Budgetrahmen, Projektumfang berücksichtigen, Auszahlungsmodalitäten, finanzielle Deckung

- Antragstellung (3 Nennungen, $2 \%$ der Befragten)

Antragstellerlnnen einbeziehen, leichtere Einreichung, verständlichere Formulierungen

- Verwertung (2 Nennungen, $2 \%$ der Befragten)

gemeinsame Plattformen und Veranstaltungen

In der dritten Frage konnten die positiven Erfahrungen, die im Rahmen der Projekte gemacht wurden, mitgeteilt werden. Hier haben insgesamt 122 RespondentInnen (67\% der Befragten) eine Angabe gemacht. Da der Großteil der Nennungen sehr ähnlich ist, wurde auf inhaltliche Gliederung bzw. Zählung der Nennungen verzichtet. Stattdessen werden ausgewählte Aussagen aufgelistet, die einen exemplarischen Einblick in die positiven Erfahrungen der Projektbeteiligten ermöglichen sollen.

- Die Begeisterung und der Forschergeist der Kinder und Jugendlichen (strahlende Kinderaugen und die große Neugier) sowie die positiven Rückmeldungen der PädagogInnen und Eltern.

- Das Kennenlernen von Persönlichkeiten aus Forschung und Entwicklung sowie der Einblick in die wissenschaftliche Arbeitswelt.

- Die Arbeit mit Kindern war eine sehr erfrischende Abwechslung von dem ansonsten von Zahlen bestimmten Arbeitsalltag.

- Die Verknüpfung verschiedener Unternehmen und Forschungseinrichtungen zu einem Bildungsauftrag ist ein vielversprechender Ansatz.

- Das Betreuungsteam der FFG ist extrem serviceorientiert. Ich kenne keine Förderstelle, die ähnlich lösungsorientiert und freundlich ist. 
Alle Projektbeteiligten, die am Anfang des Fragebogens angegeben hatten, bereits an mehreren Projekten von "Talente regional“ beteiligt gewesen zu sein, wurden gefragt, ob es zwischen den verschiedenen Ausschreibungen, Projekten bzw. Projektkonsortien erwähnenswerte Unterschiede gab. Von den 67 RespondentInnen, die an mehreren Projekten teilgenommen haben, haben insgesamt 29 (43\% der ausgewählten Stichprobe) die Möglichkeit genutzt und ihre Einschätzungen und Erfahrungen mitgeteilt. Da sich die Nennungen größtenteils sehr ähnlich sind, wurden diese nicht inhaltlich gegliedert bzw. gezählt. Stattdessen sollen einzelne exemplarische Aussagen einen Eindruck von den Erfahrungen der Projektbeteiligten vermitteln.

- Abgesehen vom Themenfeld gestalten sich die Ausschreibungen sehr ähnlich.

- Die Abrechnungen und Formulare sind einfacher geworden.

- Die längere Laufzeit ist von großem Vorteil, da längerfristige Beobachtungen und Experimente durchgeführt werden können.

- Die Abwicklung des Projekts ist einfach und effizient. Mit einem relativ geringen Aufwand (wenig "Schreibkram" für den Projektleiter) können den SchülerInnen interessante und erlebnisorientierte Experimente nähergebracht werden.

- Das Konsortium sollte nicht zu groß sein, da es sonst unübersichtlich wird.

- Die Komplexität der zu berücksichtigenden Aspekte (bspw. Migration- und Genderthematik) ist gestiegen. 


\section{Qualitative Detailanalysen}

Aufbauend auf den Ergebnissen der quantitativen Befragung wurden leitfadengestützte Interviews zur Vertiefung thematischer Schwerpunkte, die für die Weiterentwicklung bzw. Bewertung für „Talente regional" hohe Relevanz haben, durchgeführt. Die konkrete Auswahl der Leitfragen sowie der Zielgruppe wurde im Rahmen eines Meetings (mit VertreterInnen des BMVIT, der FFG und des Bewertungsgremiums) festgelegt.

Der erste Schwerpunkt richtete sich an (vor-)schulische Bildungseinrichtungen als Projektbeteiligte. Im Fokus steht die Frage, wie die Auswirkungen der Aktivitäten wahrgenommen wurden und wie die Akteurlnnen die damit einhergehenden Effekte erkennen bzw. beschreiben. Ziel ist es dabei sowohl die positiven, als auch herausfordernde Aspekte im Detail zu beschreiben. Abschließend wurde dann auch noch das komplexe Projektdesign bzw. die Vereinbarkeit der verschiedenen Schwerpunkte und Zielsetzungen thematisiert.

Der zweite Schwerpunkt richtete sich an Organisationen, die an mehreren Ausschreibungen beteiligt waren und somit über einen besonders großen Erfahrungsschatz verfügen. Hier standen die Erfolgsfaktoren sowie der Einfluss der Zusammensetzung der ProjektpartnerInnenschaft und/oder thematisch-inhaltlichen Ausrichtung (hochtechnologische Forschung / Industrie \& Produktion / alltagsbezogene Themenfelder) im Vordergrund. Abschließend wurde dann auch noch das komplexe Projektdesign bzw. die Vereinbarkeit der verschiedenen Schwerpunkte und Zielsetzungen thematisiert.

Die Kontaktdaten sowie wichtige Charakteristika (Rolle der Organisation im Projekt sowie Anzahl der Projektteilnahmen) zur Auswahl der InterviewpartnerInnen wurden bei der quantitativen Befragung erfasst. Im ersten Schwerpunkt wurde die Zielgruppe der Bildungseinrichtungen, aufgrund einer zu geringen Anzahl an Rückmeldungen, auf jene die im Rahmen von Kooperationszuschüssen beteiligt waren, ausgeweitet. Insgesamt konnten sechs PädagogInnen, drei aus Bildungseinrichtungen mit einer Projektbeteiligung und drei aus Bildungseinrichtungen mit einem Kooperationszuschuss, befragt werden. Beim zweiten Schwerpunkt konnten ebenfalls sechs Personen interviewt werden, deren Organisationen in Summe an 29 Projekten (zumeist als Konsortialführung) beteiligt waren.

\subsection{Schwerpunkt 1 - Wirkungen und Effekte}

Der Großteil der Ausführungen in diesem Abschnitt bezieht sich auf Erfahrungen von PädagogInnen aus Bildungseinrichtungen mit einer Projektbeteiligung. Eine Ausnahme bildet die Beschreibung der Wirkungen bei den Kindern und Jugendlichen (Abschnitt 5.1.3), die auch für die Aktivitäten der Kooperationszuschüsse zutreffen. Die letzten beiden Absätze im Abschnitt 5.1.1 beziehen sich ausschließlich auf Aussagen von PädagogInnen aus Bildungseinrichtungen mit einem Kooperationszuschuss.

\subsubsection{Wirkungen auf die Bildungseinrichtung}

Die Passung mit dem Lehrplan stellt für die Befragten zumeist keine Herausforderung dar. Es wird angemerkt, dass die interdisziplinäre Herangehensweise im Rahmen der verschiedenen Unterrichtsfächer als äußerst zielführend und bereichernd wahrgenommen wird. Die ExpertInnen eröffnen den Kindern und Jugendlichen unterschiedliche Blickwinkel und gewährleisten somit eine intensive und vielschichtige Auseinandersetzung mit der jeweiligen Materie. Vorteilhaft ist es allerdings, wenn die fachliche Übertragbarkeit bei der Wahl und Konzeption des thematischen Schwerpunkts berücksichtigt wird, da es sonst für die PädagogInnen sehr aufwändig ist, den fächerübergreifenden Unterricht untereinander abzustimmen. 
Als besonders wirkungsvoll werden die Exkursionen sowie Besichtigungen von Organisationen aus der Forschung und Wirtschaft eingeschätzt. Da man in diesem Rahmen die Möglichkeit hat, die Infrastruktur vor Ort zu nutzen und dadurch die Übungen und Experimente einen stärkeren Praxisbezug aufweisen. Außerdem können alle Teilnehmerlnnen bei den Aufgaben wirklich selbst anpacken und aufgrund des Know-hows der ExpertInnen sind die Versuchsabläufe zumeist sehr detailliert und durchdacht. Weiters können die Methoden und Erfahrungen von den PädagogInnen in den alltäglichen Unterricht aufgenommen werden und auch der Kontakt zu den besuchten Organisationen bleibt in den meisten Fällen erhalten.

Diese Zusammenarbeit kann in Folge den Zugang zu weiteren Kontakten bzw. Netzwerken ermöglichen, wodurch die Suche neuer regionaler KooperationspartnerInnen erleichtert wird. Aber auch die Vernetzung mit anderen Bildungseinrichtungen hat einen ähnlichen Effekt und begünstigt ebenfalls die Bildung von Kooperationen zwischen den Bereichen Schule, Forschung und Wirtschaft. So kommen die höheren Schulen im Rahmen der Projekte mit den SchülerInnen der vorgelagerten Schulstufen in Kontakt, wodurch sich oftmals auch die Möglichkeit ergibt, die Räumlichkeiten des Standortes vor Ort besser kennenzulernen. Somit können die Schulen das eigene Profil den potenziellen Schülerlnnen gezielt näherbringen und stärken gleichzeitig auch die Sichtbarkeit und Relevanz des eigenen Schulstandortes in der Region.

Um die schulinterne Koordination zu meistern, gab es in den Schulen oft eine zentrale Ansprechperson, die die Verantwortung für die Organisation übernimmt und versucht die Aufgaben und Informationen im Kollegium zu verteilen. Diese treibt auch die Initiative vor Ort voran und bildet somit die Schnittstelle zwischen dem Projektkonsortium und der Bildungseinrichtung.

In Bezug auf die Wirkungen der Projektteilnahme auf die Schulentwicklung wird konstatiert, dass durch die Aktivitäten im Bereich Naturwissenschaft und Technik die Attraktivität des jeweiligen Schulstandortes grundsätzlich gesteigert wird. Einerseits durch die Verbreiterung der schulinternen Angebotspalette und andererseits durch die verstärkte Professionalisierung des Lehrpersonals.

So fördert die Beteiligung in vielen Fällen die Motivation der PädagogInnen an Schulungen und Seminaren teilzunehmen, da sie das Gelernte gleich direkt in der Praxis anwenden können. Teilweise bilden sich auch lokale Initiativen oder Programme, in denen versucht wird, die erlernten Erkenntnisse und Methoden mit Hilfe von kollegialem Mentoring weiterzugeben.

Es gibt auch Projekte, in denen die Ergebnisse der Aktivitäten zu baulichen Veränderungen im Erscheinungsbild der Schule geführt haben und somit den schulischen Alltag auch räumlich prägen. Dabei handelt es sich bspw. um vertikale Begrünungen oder ein Labor mit Materialien bzw. Geräten für Übungen und Experimente. Außerdem können die unterschiedlichen Maßnahmen und Aktivitäten auch als Grundlage für Zertifizierungen oder Auszeichnungen herangezogen werden.

Allerdings wird darauf hingewiesen, dass vor allem Schulen an Aktivitäten teilnehmen, die ohnehin sehr aktiv sind. Sprich zu einer grundlegenden Veränderung der Sichtweise bzw. Kultur in der Schule kommt es laut den Befragten nicht unbedingt. Man sieht die Teilnahme vielmehr als zusätzliche Ressource, mit deren Hilfe man das bereits existierende Engagement an den Schulen fördern und unterstützen kann.

Bei den PädagogInnen, die sich ausschließlich auf die Aktivitäten im Rahmen eines Kooperationszuschusses beziehen, wird die zentrale Bedeutung der finanziellen Unterstützung zum Aufbau der schulischen Infrastruktur betont. Dabei handelt es sich zumeist um spezielle Geräte, ForscherInnenboxen oder unterstützende Literatur. Diese sind notwendig, um langfristig praxisbezogene Übungen und Experimente im Klassenzimmer durchführen zu können. Weiters fördert der Kooperationszuschuss die Motivation der PädagogInnen Exkursionen und Ausflüge anzubieten, da die Finanzie- 
rung der Reise- bzw. Fahrtkosten gewährleistet ist und der Organisationsaufwand entsprechend geringer ist.

Die Inanspruchnahme der Kooperationszuschüsse ist relativ unkompliziert, wodurch vor allem innovative Ideen und Initiativen, die oftmals an der Finanzierung und dem damit einhergehenden Aufwand scheitern, gefördert werden. Im Vergleich zu anderen Förderungen wird der Multiplikatoreffekt der eingesetzten Mittel als extrem hoch eingeschätzt.

\subsubsection{Störfaktoren und Herausforderungen}

Beispiele für Störfaktoren auf der Ebene der Bildungseinrichtung, die im Rahmen der Aktivitäten auftreten können, konnten von den befragten PädagogInnen nicht genannt werden. Allerdings gibt es durchaus zentrale Faktoren, die berücksichtigt werden müssen, um eine friktionsfreie Umsetzung der Aktivitäten gewährleisten zu können.

Ein zentraler Faktor für die Bildungseinrichtungen ist die thematische Passung der Aktivitäten sowie die Übertragbarkeit der Inhalte auf die jeweilige Fächerverteilung der einzelnen Schulstufe. Laut den PädagogInnen müssen die Inhalte einerseits sehr gut vorstrukturiert und aufbereitet sein, und andererseits frühzeitig abgestimmt werden, um diese erfolgreich in den Lehrplan bzw. Unterricht integrieren zu können. Dabei sollte auch der jeweilige Wissensstand der Teilnehmerlnnen berücksichtigt werden, um darauf aufbauend eine altersgerechte Vermittlung der Informationen gewährleisten zu können.

Besonders vorteilhaft ist es, wenn die zuständigen Personen den Projektbeteiligten selbst einen pädagogischen Hintergrund aufweisen und konkrete Erfahrungen im Umgang mit Kindern und Jugendlichen mitbringen. Im Idealfall sollte also die Auswahl der Projektbeteiligten auch auf die Charakteristika der verschiedenen Schulformen und deren Schwerpunkte abgestimmt sein. Dies funktioniere am besten, wenn sich die Konsortialführung nicht nur auf die administrativen und organisatorischen Aufgaben fokussiert, sondern auch bei der thematischen und didaktischen Umsetzung ihre Expertise einbringt.

Die Terminfindung bzw. -koordination wird in diesem Zusammenhang als besonders anspruchsvoll eingeschätzt, da das Schuljahr bzw. der Lehrplan schon lange (zumeist ein Jahr) im Voraus geplant wird und somit Änderungen zu einem enormen Mehraufwand führen können. Dies trifft vor allem auf die höheren Schulstufen zu, die mit der Vielzahl an Terminen und Verpflichtungen (mehrstündige Schularbeiten, vorwissenschaftliche Arbeit, standardisierte Reifeprüfung etc.) ohnehin schon ausgelastet sind. Aus diesem Grund sind die PädagogInnen der Meinung, dass man sich vor allem auf die niedrigeren Schulstufen, unter der Sekundarstufe 2, konzentrieren sollte.

Weiters sollte man sich als Bildungseinrichtung im Vorfeld ganz konkret darüber Gedanken machen in welchem Umfang die Aktivitäten überhaupt umgesetzt werden können. Es empfiehlt sich in diesem Zusammenhang alle Projektbeteiligten bezüglich der Aufgaben und der Zuständigkeiten zu sensibilisieren und bei Bedarf auch entsprechende Einschränkungen vorzunehmen. Gerade wenn der zeitliche Rahmen relativ großzügig bemessen wird, der für die erfolgreiche Umsetzung der Maßnahmen notwendig ist, wird der tatsächliche Arbeitsaufwand oftmals unterschätzt.

Neben den organisatorischen Aspekten ist auch die Moral der am Projekt Beteiligten von großer Bedeutung. Ohne einem hohen Grad an Begeisterung und Motivation ist es nicht möglich, den mit den Projekten einhergehenden Ansprüchen gerecht zu werden. Viele PädagogInnen sind zu Beginn auch eher zögerlich, weil mit den Aktivitäten natürlich ein gewisser Mehraufwand einhergeht. Nur mit Hilfe von regelmäßigen Meetings und viel Überzeugungsarbeit ist es möglich, diese Bedenken schrittweise abzubauen. Es wird auch betont, dass es im schulischen Altag an entsprechenden 
„Freiräumen“ mangelt, sowohl zeitlich als auch fachlich. Dazu kommt noch, dass es für die Pädagoglnnen in diesem Setting wenig Anreize gibt, sich an Projekten zu beteiligen.

\subsubsection{Wirkungen auf die Kinder und Jugendlichen}

Der zentrale Effekt ist, laut den PädagogInnen, die Steigerung des Interesses für Inhalte und Themen, die im Regelunterricht zumeist eher unbeliebt sind. Dies ist vor allem auf den Einsatz vielfältiger Methoden, Ansätze und Perspektiven zurückzuführen, die es ermöglichen die Faszination der gewählten Materie für Kinder und Jugendliche greifbarer zu machen. Im Vordergrund stehen hier nicht nur die durchdachte Konzeption der Experimente und Übungen sondern auch die abwechslungsreiche Aufbereitung sowie die interdisziplinäre Übertragbarkeit.

Vor allem die verschiedenen Besichtigungen und Exkursionen, die einen klaren Bruch zum herkömmlichen Schulalltag darstellen, werden als besonders bereichernd wahrgenommen. Die TeilnehmerInnen entwickeln im Rahmen der Aktivitäten eine große Begeisterung und haben die Möglichkeit sich relativ unbefangen einer Thematik zu widmen. Den Kindern und Jugendlichen wird gezeigt, welche Konsequenzen das eigene Verhalten in dem jeweiligen Kontext haben kann und wie eng die abstrakten Phänomene und Mechanismen mit ihrem sozialen und regionalen Umfeld verbunden sind.

Das Projektsetting bildet eine wichtige Voraussetzung, um diese methodische Offenheit zu gewährleisten, da in diesem Rahmen die grundsätzliche Bereitschaft etwas Neues auszuprobieren relativ groß ist und somit eher gängige Muster aufgebrochen werden können. Für die PädagogInnen sind die Aktivitäten sowohl eine willkommene Abwechslung, als auch eine wertvolle Ressource, auf deren Grundlage viele Kontakte geknüpft werden sowie Informationen gesammelt werden können.

Neben der Steigerung des Interesses bzw. der Erweiterung der fachlichen Kenntnisse wird auch der positive Effekt auf die Entwicklung des Selbstbildes der Kinder und Jugendlichen betont. Die TeilnehmerInnen begreifen durch die direkte Resonanz der eigenen Handlung erst wozu sie eigentlich in der Lage sind. Damit sich dieses Vertrauen auch manifestiert, müssen die Ergebnisse entsprechend verwertet bzw. sichtbar gemacht werden. Erst durch die externe Aufmerksamkeit bzw. Bestätigung bekommen die eigenen Leistungen eine gewisse Relevanz und werden somit ernst genommen, wodurch die Teilnehmerlnnen ermutigt werden, ihr Potenzial zu entfalten. Dieser Prozess ist, laut den PädagogInnen, enorm entscheidend, da prinzipiell alle Kinder und Jugendlichen ein gewisses Interesse mitbringen, aber das eigene Selbstvertrauen dafür ausschlaggebend ist, ob man sich auch mit unbekannten oder anspruchsvollen Inhalten auseinandersetzt.

Besonders relevant ist dieser Aspekt im Kontext der geschlechtsspezifischen Bildungswahl aber auch der Integration von bildungsfernen Bevölkerungsgruppen. In beiden Fällen ist es wichtig, die Barrieren und Ängste abzubauen, damit sich die Kinder und Jugendlichen entsprechend ihrer Neigungen und Talente entwickeln können. Hier können beispielsweise der Kontakt mit unterschiedlichen Berufsbildern und/oder Role-Models den Horizont der Kinder entscheidend erweitern.

Eine ähnliche Wirkung haben, laut den PädagogInnen, die verschiedenen Peer-Mentoring/TutoringKonzepte. Die jüngeren Schülerlnnen genießen die ungeteilte Aufmerksamkeit und exklusive Betreuung und können somit bei der Umsetzung von anspruchsvolleren Aufgabenstellungen begleitet werden. Für die älteren SchülerInnen ist hingegen der Wechsel in die Perspektive des Lehrenden besonders eindrucksvoll, da sie einerseits den Respekt der jüngeren SchülerInnen, aber auch einen Einblick in die anspruchsvolle Rolle der PädagogInnen bekommen. Dies steigert wiederum das eigene Selbstwertgefühl und stärkt das Verständnis und die Wertschätzung für die Arbeit der Lehrenden. 


\subsubsection{Reflexionen zum komplexen Projektdesign}

Grundsätzlich sind die PädagogInnen der Meinung, dass die verschiedenen Zielsetzungen im Rahmen der Projektkonzeption zumeist gut vereinbar und umsetzbar waren. Lediglich bei den Schwerpunkten Geschlechterausgewogenheit und Integration von Kindern und Jugendlichen unabhängig ihrer sozialen oder geographischen Herkunft ist man zum Teil etwas verunsichert, da es sich um sehr abstrakte Themen handelt, die im Rahmen der Aktivitäten nicht immer integriert werden können.

Die PädagogInnen weisen darauf hin, dass die Angaben im Rahmen der Ausschreibung zu allgemein formuliert und die Begriffe nicht ausreichend operationalisiert werden. Dadurch fehlt es zum Teil an projektspezifischen Anknüpfungspunkten, die die Auswahl der passenden Methoden sowie des gewünschten Kontextes erleichtern würden. Aber auch in der konkreten Umsetzung ergeben sich aufgrund des lokalen Umfelds verschiedene Beschränkungen. So ist es nicht in jeder Region möglich die passenden Role-Models zu finden oder die gleichmäßige Verteilung der Geschlechter bei den ExpertInnen zu erreichen.

Nichtsdestotrotz wird die Umsetzung dieser Schwerpunkte als äußerst wichtig und erstrebenswert erachtet und man unterstützt die Bemühungen die Sensibilisierung in diesen Themenbereichen weiter voranzutreiben. Allerdings sollte der Anspruch bzw. die Erwartungshaltung in diesem Kontext stärker an die Bedingungen in den Bildungseinrichtungen angepasst werden. Die Teilnehmerlnnen werden im Rahmen der Aktivitäten mit einer Flut an Informationen versorgt und die Themen Geschlechterausgewogenheit und Integration von Kindern und Jugendlichen unabhängig ihrer sozialen oder geographischen Herkunft gehen teilweise unter.

Wesentlich besser kann die interkulturelle und soziale Wahrnehmung der Kinder und Jugendlichen durch den direkten Kontakt mit möglichst unterschiedlichen Personengruppen erweitert werden. Die Teilnehmerlnnen können dadurch verschiedene Perspektiven und Kulturen kennenlernen und dann die Erlebnisse und Erfahrungen auch im eigenen Bekanntenkreis besprechen. Außerdem helfen viele der angewandten Methoden, wie bspw. das spielerische Lernen, grundsätzlich dabei die Scheu vor technischen und naturwissenschaftlichen Inhalten abzulegen und unterstützen die TeilnehmerInnen unabhängig von Geschlecht, Nationalität oder sozialer Schicht. 


\subsection{Schwerpunkt 2 - Erfolgsfaktoren}

Der zweite Schwerpunkt richtete sich an Organisationen, die an mehreren Ausschreibungen beteiligt waren und somit über einen besonders großen Erfahrungsschatz verfügen. Die folgenden Ausführungen beziehen sich auf die Angaben von fünf Befragten, deren Rückmeldungen zwar verschiedene Teilbereiche in den Vordergrund stellen, sich aber bei der inhaltlichen Bewertung der Aktivitäten und Maßnahmen zum Großteil decken. Lediglich bei der Vereinbarkeit der Zielsetzungen (erster Unterpunkt in Abschnitt 5.2.6) unterscheiden sich die Rückmeldungen dieser Befragten bezüglich der Themen Geschlechterausgewogenheit und der Integration von Kindern und Jugendlichen unabhängig ihrer sozialen oder geographischen Herkunft etwas voneinander.

Der letzte Unterpunkt in Abschnitt 5.2.6 (Kritische Betrachtung der Rentabilität) bezieht sich auf die Angaben einer befragten Person, deren Rückmeldung sich ausschließlich auf diese Thematik fokussierte.

\subsubsection{Themenfindung und Projektkonsortium}

Entscheidend ist in erster Linie, dass Themengebiete und Inhalte auswählt werden, die eine langfristige Relevanz besitzen und somit entscheidende Impulse für die spätere Bildungs- bzw. Berufswahl liefern können. Dabei sollte allerdings die Übertragbarkeit der zum Teil relativ abstrakten Inhalte in den Alltag, sowie die Lebenswelt der Kinder und Jugendlichen nicht vernachlässigt werden.

Die persönliche Betroffenheit und konkrete Anwendbarkeit ist in diesem Zusammenhang äußerst wichtig, um das Interesse und die Aufmerksamkeit der TeilnehmerInnen sicherzustellen. Dabei muss ein Spagat zwischen der Darstellung der Komplexität der wissenschaftlichen Zusammenhänge sowie der altersgerechten und praxisorientierten Vermittlung gefunden werden.

Um dies zu gewährleisten wird ein dichtes Netzwerk an passenden Kontakten zu wirtschaftlichen und wissenschaftlichen Einrichtungen, die die notwendige Expertise in den gewählten Themengebieten mitbringen, benötigt. Im besten Fall kooperieren diese Organisationen bereits miteinander und das eigene Projekt kann zum Teil auf diese Projektressourcen zurückgreifen. Dies wirkt sich äußerst positiv auf die Qualität der Aktivitäten aus, da die TeilnehmerInnen neben den fachlichen Informationen die Zusammenarbeit von Wirtschaft und Forschung in der Praxis miterleben können und dadurch die Charakteristika und Funktionsweisen beider Sphären kennenlernen.

Wichtig ist auch, dass die jeweiligen ExpertInnen Erfahrungen in der Arbeit mit Kindern mitbringen. Denn die altersgerechte Vermittlung (bspw. durch Einsatz von altersgerechter Sprache sowie der aktiven Mitgestaltung der Teilnehmerlnnen) der naturwissenschaftlichen und technischen Themen ist überaus anspruchsvoll und benötigt neben fachlichen vor allem auch pädagogische Kompetenzen.

\subsubsection{Konzeption und Angebotspalette}

Ein entscheidender Faktor ist die interdisziplinäre Umsetzung in den Projekten. Je breiter eine Thematik behandelt werden kann, desto leichter ist es für die Kinder und Jugendlichen einerseits einen persönlichen Bezug herzustellen und andererseits die vielfältigen Zusammenhänge zwischen den unterschiedlichen Disziplinen und Fächern wahrzunehmen. Hierbei sollte darauf geachtet werden, dass die Aktivitäten auf die Bedürfnisse und Schwerpunkte der Schulen und Klassen abgestimmt sind und im Einklang mit dem jeweiligen Lehrplan stehen.

Besonders vorteilhaft ist eine breite Palette an modularen Angeboten bzw. Formaten (Workshops, Exkursionen, Übungen und Experimente), die es den Bildungseinrichtungen ermöglicht, zwischen verschiedenen Aktivitäten zu wählen. Dies erhöht die Teilnahmebereitschaft von Seiten der Pädago- 
glnnen enorm, da diese dann besser steuern können, in welchem Umfang und in welcher Form sie aktiv werden möchten.

Auch die Relevanz der verschiedenen Meetings und Events, wie bspw. die Abschlussveranstaltung, sollte nicht unterschätzt werden. In diesem Rahmen können zum Projektende die Projektbeteiligten und TeilnehmerInnen an den Aktivitäten nochmals ihre Konzepte und Ergebnisse präsentieren.

\subsubsection{Einbindung und Unterstützung der Bildungseinrichtungen}

Für eine erfolgreiche Umsetzung der Projekte sollte die Kontaktaufnahme zu den Bildungseinrichtungen auf keinen Fall unterschätzt werden. Wenn kein Netzwerk an passenden Kontakten vorhanden ist, kann sich diese relativ aufwändig und zeitintensiv gestalten. Es ist zwar möglich, die Projekte zentral, bspw. über den Landesschulrat oder die Direktorlnnenkonferenz, zu bewerben. Die Erfahrung hat gezeigt, dass die direkte Kontaktaufnahme mit den PädagogInnen am besten funktioniert. Für die Konsortialführung ist es in diesem Rahmen einerseits leichter die Verantwortlichen gezielt anzusprechen und somit von der Idee des Projektes zu überzeugen, und andererseits kann auch die Motivation sowie das Engagement vor Ort besser eingeschätzt werden. Durch den persönlichen Kontakt können etwaige Anpassungswünsche besser kommuniziert und Zweifel einfacher ausgeräumt werden.

Zur konkreten Umsetzung der Projektaktivitäten benötigen die PädagogInnen die schulinterne Unterstützung durch die Direktion und dem gesamten Lehrpersonal. Das ist notwendig, damit die Aufgaben entsprechend verteilt und die Aktivitäten fächerübergreifend umgesetzt werden können. Grundsätzlich ist es aus organisatorischer Sicht sinnvoll, mehrere Lehrkräfte einzubinden, da man sonst bei der Planung und Durchführung der Aktivitäten eingeschränkt ist und auch die Belastung oftmals zu groß wird. Aus diesem Grund empfiehlt es sich, die Bildungseinrichtungen so früh als möglich in die Planung und Konzeption der Projektaktivitäten einzubinden, da deren Kooperation entscheidend für den Erfolg der Umsetzung ist. Vor allem die regelmäßige Kommunikation in Form von Feedbackgesprächen bzw. kleineren Meetings ist äußerst wichtig, um einen reibungslosen Projektablauf zu gewährleisten.

Da die Qualifikation des Lehrpersonals für die Umsetzung der Aktivitäten eine besonders wichtige Rolle spielt, sollten unbedingt projektspezifische Fortbildungen, die den Fokus auf naturwissenschaftliche und technische Themen sowie deren konkrete Vermittlung im Unterricht legen, angeboten werden. Denn gerade die Einbettung der Weiterbildung in den jeweiligen Kontext des Projektes ermöglicht es den PädagogInnen das Gelernte gleich direkt in den geplanten Aktivitäten umzusetzen und gegebenenfalls um eine didaktische Unterstützung anzusuchen. Außerdem ist der langfristige Nutzen dieser Weiterbildungen enorm groß, da die im Projekt entstandenen Erfahrungen somit leichter in den Schulalltag integriert werden können und damit deren nachhaltige Wirkung gefördert wird.

Die Fortbildungen können bspw. in einem didaktisch-fachlichen Tandem durchgeführt wurden, in dem zwei ExpertInnen gemeinsam ihre Methoden und Übungen in Kleingruppen vermitteln. Die ReferentInnen für den didaktischen Teil kommen zumeist von einer pädagogischen Hochschule und der fachliche Teil wird von Vertreterlnnen der wissenschaftlichen oder wirtschaftlichen ProjektpartnerInnen übernommen. Durch dieses besondere Betreuungsverhältnis kann die intensive didaktische und fachliche Auseinandersetzung mit den Inhalten garantiert werden. In diesem Rahmen können neue Experimente und Übungen sowie zeitgemäße Materialien und Methoden, die sich am aktuellen Forschungsstand orientieren, in den Unterricht aufgenommen werden. Dadurch finden vermehrt innovative Ansätze den Weg ins Klassenzimmer und ersetzen dort veraltete und zum Teil nicht mehr zeitgemäße Praktiken. 


\subsubsection{Unterschiede in Bezug auf Zusammensetzung und Ausrichtung}

Grundsätzlich wird die Meinung vertreten, dass es bezüglich der Erfolgsfaktoren keine nennenswerten Unterschiede zwischen den jeweiligen Zusammensetzungen sowie der thematischen Ausrichtung in den verschiedenen Projektbeteiligungen gab. Allerdings gibt es durchaus gewisse Differenzen in der Schwerpunktsetzung, die im Rahmen der unterschiedlichen Projekte beachtet werden sollten.

Bei der Vermittlung von Themengebieten mit einem hohen Grad an Exklusivität oder Innovationskraft steigt der Anspruch an die fachlichen und pädagogischen Kompetenzen der Projektbeteiligten. Dafür braucht es spezifische Schulungen, in denen die ExpertInnen und Lehrkräfte wertvolle didaktische und fachliche Inputs bekommen. Ohne das entsprechende Rüstzeug ist die Umsetzung der geplanten Maßnahmen nur in einem beschränkten Ausmaß möglich.

Hinzu kommt, dass die Übertragbarkeit der Inhalte bei niedrigeren Schulstufen und speziell im Kindergarten, auf Grund des Alters der TeilnehmerInnen und des damit einhergehenden Wissenstandes, als sehr anspruchsvoll eingeschätzt wird. In den höheren Schulstufen gestaltet sich die Vermittlung zumeist leichter, weil bereits grundlegende Kenntnisse vorhanden sind und es teilweise auch Kooperationen mit Fachhochschulen oder Universitäten gibt, die ihr Know-how zur Verfügung stellen.

Ein weiteres Unterscheidungskriterium ist der Grad der Normierung des Projektes. So gibt es auf der einen Seite Projekte, die einen klar abgegrenzten Rahmen mit aufeinander aufbauenden Modulen haben, und andere mit sehr unterschiedlichen Aktivitäten, wo die Anknüpfungspunkte untereinander eher gering sind. Dies kann sowohl der schulinternen Ressourcen (bspw. fehlende Geräte und Materialen oder zu wenig Lehrpersonal), als auch den Präferenzen geschuldet sein. Es gibt sowohl Bildungseinrichtungen, die sich in gewissen Bereichen keine Veränderungen zutrauen und sich vor allem an zentralen Vorgaben orientieren, als auch andere, die wesentlich autarker handeln und somit offen sind für unterschiedliche Aktivitäten.

\subsubsection{Indikatoren für den Erfolg}

Die Befragten geben an, dass versucht wurde, den Erfolg der Projektaktivitäten durch Evaluierungen sichtbar zu machen. Dabei wurden sowohl die Teilnehmerlnnen, als auch die Vertreterlnnen der verschiedenen Projektbeteiligten befragt. Allerdings ist es aufgrund der Vielzahl und des zumeist qualitativen Charakters der verschiedenen Effekte äußerst schwierig, diese valide zu messen. Hinzu kommt auch noch die Problematik, dass man die Veränderungen bei den TeilnehmerInnen über einen längeren Zeitraum beobachten müsste, um aussagekräftige Aussagen über den Erfolg machen zu können.

Als Erfolgsfaktoren werden von den Befragten vor allem qualitative Kriterien, die im Rahmen von persönlichen Rückmeldungen kommuniziert werden, genannt. Besonders der Stolz der Kinder und Jugendlichen auf die eigene Leistung und die Motivation ist unübersehbar, wenn sie ihre Ergebnisse im Rahmen einer Abschlussveranstaltung präsentieren können. Dadurch bekommt der Einsatz der TeilnehmerInnen eine andere Wertigkeit, weil sie dann sehen, wie ihre erstellten Werkstücke oder Materialien betrachtet werden. Das stärkt das Selbstbewusstsein und die Eigeninitiative der Kinder und Jugendlichen und ist die Basis dafür, die Scheu vor naturwissenschaftlichen oder technischen Themen abzubauen.

Optimal ist es, wenn die TeilnehmerInnen dazu motiviert werden, ihre eigenen Ideen und Vorstellungen einzubringen und bspw. die Versuchsanleitung eines Experiments durch kleine Adaptionen zu erweitern. Dies ist ein Beleg dafür, dass die Kinder und Jugendlichen die Thematik verstanden haben und auch das Interesse sich damit intensiver auseinanderzusetzen geweckt wurde. In vielen Fällen 
werden diese Erfahrungen dann auch mit den Eltern und dem Bekanntenkreis geteilt, was wiederum den Erfolg der Aktivität wiederspiegelt.

Die Implikationen für die spätere Bildungs- bzw. Berufswahl sollte nicht unterschätzt werden, da in vielen Maßnahmen Exkursionen und Besichtigungen durchgeführt werden, die den Einblick in das Umfeld und die Tätigkeiten verschiedener Berufsfelder ermöglichen.

Die Tatsache, dass viele der beteiligten Organisationen sich immer wieder an neuen Projekten beteiligen, die Aktivitäten von den Bildungseinrichtungen gerne in Anspruch genommen werden und es auch eine anhaltende Nachfrage der PädagogInnen nach Fortbildungen gibt, zeigt, dass die geförderten Maßnahmen eine hohe Wertschätzung genießen. Im Rahmen der Projekte haben sich im Zeitverlauf viele intensive Kooperationen zwischen Schulen, Unternehmen und Universitäten gebildet, an denen auch zukünftige Projekte andocken können. Im Rahmen der Projektteilnahmen wurden sogar eigene Vereine gegründet, deren Wirkungsbereiche weit über die ursprüngliche Projektidee hinausgehen. Diese etablierten Strukturen sind ein wichtiger Indikator für den Erfolg der Förderungen und zeigen, dass die Investitionen einen großen Mehrwert haben, deren Wirkung anhält.

\subsubsection{Reflexionen zum komplexen Projektdesign}

Die verschiedenen Aspekte zum komplexen Projektdesign beziehen sich auf relativ unterschiedliche Abstraktionsebenen und können somit nur schwer miteinander in Verbindung gebracht werden. Daher wurde nur eine grobe Gliederung einzelner Themen vorgenommen, die nicht aufeinander aufbauen und sich hauptsächlich auf den jeweiligen Teilaspekt konzentrieren.

\section{Vereinbarkeit der Zielsetzungen}

Die Befragten gaben an, dass die verschiedenen Zielsetzungen im Rahmen der Projekte grundsätzlich gut vereinbar und umsetzbar waren. Von PädagogInnen werden die Ziele der Geschlechterausgewogenheit sowie der Integration von Kindern und Jugendlichen unabhängig ihrer sozialen oder geographischen Herkunft bei der Umsetzung der Projekte als besonders herausfordernd und teilweise sogar als störend wahrgenommen.

Zum Teil können die Vorgaben in diesen Bereichen, wenn bei der Wahl der Methoden und Konzepte die regionalen und sozialen Gegebenheiten berücksichtigt werden, durchaus im gewünschten Ausmaß umgesetzt werden. Der gezielte Einsatz von Role-Models oder sprachsensiblen Unterrichtsmethoden werden als überaus erfolgreich eingeschätzt.

Allerdings sind ein Teil der Befragten auch der Meinung, dass diesen Themen zu viel Aufmerksamkeit geschenkt wird und der Fokus sich stärker auf naturwissenschaftliche und technische Inhalte und nicht auf politische oder gesellschaftliche Fragestellungen richten sollte. Man sollte bei den Schwerpunkten mehr Wert auf die Prioritätensetzung legen, da sich diese Punkte in gewisser Weise auf Basis der anderen Zielsetzungen ergeben sollten und daher nicht so prominent und explizit angesprochen werden müssen. In den meisten Fällen zielen die Aktivitäten und Übungen ohnehin darauf ab, die Zielgruppe ganz unabhängig von gewissen Merkmalen zu fördern und anzusprechen.

Weiters sollte nicht unterschätzt werden, dass man bei der Adressierung und Umsetzung dieser Thematik stark von der Bereitschaft und Qualifikation der jeweiligen Lehrkraft vor Ort abhängig ist. Hinzu kommt auch noch die Tatsache, dass in Kindergärten und Volksschulen das Lehrpersonal fast ausschließlich weiblich ist. Diese strukturelle Gegebenheit prägt die Kinder wesentlich stärker als punktuelle Maßnahmen und Aktivitäten. Außerdem wird der fast ausschließliche Fokus der Gendersensibilität auf die Rolle der Frau als zu einseitig empfunden und angeraten, diese durch die Vielseitigkeit der männlichen Rolle zu ergänzen. 
Weitere Kritikpunkte sind der Grad der Operationalisierung der Begriffe sowie zu wenig konkrete Formulierungen wie die Vorgaben integriert bzw. umgesetzt werden sollen.

\section{Zusammenarbeit mit den Bildungseinrichtungen}

Im Rahmen der verschiedenen Projektteilnahmen hat sich gezeigt, dass die Zusammenarbeit mit kleineren Bildungseinrichtungen sowie niedrigeren Schulstufen meist einfacher ist, da die Organisation schlanker ist und Abstimmungen rascher durchgeführt werden können.

Man konnte ebenfalls beobachten, dass die Schulen in ländlichen Gegenden zumeist besser ausgestattet sind. Dies betrifft sowohl die Infrastruktur wie Geräte und Materialien, als auch die personellen Ressourcen. Außerdem weisen diese Schulen auch eine höhere Teilnahmebereitschaft auf als größere Schulen in Ballungszentren. Die Bildungseinrichtungen in größeren Städten erhalten teilweise sehr viele Projektanfragen und sind deswegen oft überfordert, die Fülle an Angeboten überhaupt in Anspruch zu nehmen. Je weniger regionale Angebote es gibt, desto größer ist zumeist auch das Interesse an den Aktivitäten teilzunehmen.

\section{Einbindung der Eltern}

Ein Aspekt, der sich im Rahmen der Aktivitäten nur schwer bzw. gar nicht umsetzen ließ, ist die direkte Einbindung bzw. Beteiligung der Eltern. Aufgrund der Erwerbstätigkeit der meisten Eltern ist es für diese kaum möglich an Schulaktivitäten, die meistens am Vormittag stattfinden, teilzunehmen. Allerdings wurde die Erfahrung gemacht, dass es sehr gut funktioniert die Eltern über die Kinder und Jugendlichen über das regionale Angebot an wissenschaftlichen Einrichtungen und Unternehmen zu informieren bzw. zu sensibilisieren. Diese Strategie funktioniert sehr gut, weil die Teilnehmerlnnen fasziniert sind und die erhaltenen Informationen gerne verbreiten und somit auch die Möglichkeit haben in die Rolle der Informationsvermittlung zu schlüpfen.

Darüber hinaus wurden den Kindern auch kleinere Übungen bzw. Experimente gegeben, um diese gemeinsam mit ihren Eltern durchzuführen. Für einige Mädchen ist dies besonders spannend, wenn sie mit naturwissenschaftlichen bzw. technischen Fragestellungen ihren Vater ansprechen können.

\section{Kooperationszuschüsse}

Mit den Kooperationszuschüssen kann man die Bildungseinrichtungen in der Umgebung sehr gut ansprechen und verbreitert dadurch die regionale Bekanntheit der Projekte entscheidend. Die finanzielle Unterstützung ermöglicht es den PädagogInnen die Infrastruktur (Geräte und Materialien) an den Bildungseinrichtungen auszubauen und fördert das Interesse an Exkursionen oder Besichtigungen teilzunehmen. Die Deckung der Fahrtkosten ist zumeist mit einem relativ hohen organisatorischen Aufwand verbunden, der die Umsetzung einzelner Maßnahmen entscheidend hemmen kann. Durch diese Entlastung kann die Experimentierfreudigkeit der PädagogInnen gestärkt und die Teilnahme von Kindern und Jugendlichen, unabhängig vom sozialen Hintergrund, erleichtert werden.

Außerdem entstehen daraus auch Kooperationen zu verschiedensten Organisationen aus Wirtschaft und Forschung. Aufbauend auf der Zusammenarbeit mit diesen Kontakten und den daraus resultierenden Erfahrungen können die Bildungseinrichtungen dann leichter für eine zukünftige Projektteilnahme gewonnen werden.

\section{Aufbau nachhaltiger Strukturen}

Um die Effekte und Ergebnisse, die im Rahmen der Projekte erzielt werden konnten, langfristig noch besser verwertbar zu machen wird angeraten, die etablierten Strukturen über die Projektlaufzeit hinaus weiter zu fördern. Dabei geht es weniger um eine Finanzierung von bestimmten Maßnahmen oder Aktivitäten, sondern um die öffentliche Unterstützung von regionalen Anlaufstellen. Diese sind notwendig, um das bestehende Angebot zu koordinieren sowie zu bewerben und den Fortbestand 
besonders nachhaltiger Maßnahmen, wie bspw. Fortbildungsprogramme für Pädagoglnnen, zu gewährleisten. Dadurch können vor allem die Kontakte zu den Bildungseinrichtungen intensiviert werden, was sich wiederum positiv auf die Kommunikationsbasis, den Informationsstand sowie die Akzeptanz an Maßnahmen teilzunehmen, auswirken sollte. Nur wenn Strukturen und Angebote über einen längeren Zeitraum bereitgestellt werden können, kommt es laut den Befragten zu nennenswerten Netzwerkeffekten.

\section{Kritische Betrachtung der Rentabilität}

Neben den durchwegs positiven Rückmeldungen zu den Projekten und deren Konzeption, äußerte sich auch eine der befragten Personen überaus kritisch, vor allem in Bezug auf die Rentabilität der eingesetzten Fördermittel. Diese ist der Meinung, dass die Projekte einfach in einem zu großen Rahmen stattfinden und dadurch zu viele Ressourcen in die Organisation, Koordination und Dokumentation fließen würden. In Relation zum eingesetzten Kapital werden die Mittel, die bei der eigentlichen Zielgruppe ankommen, als zu gering eingeschätzt. Demzufolge ist auch der Anspruch der Projekte, bspw. durch die zu starke Orientierung am Innovationsgrad, zu hoch angesetzt wird, was dazu führt, dass die Kinder und Jugendlichen gar nicht in der Lage sind, die Inhalte zu verarbeiten.

Bemängelt wird zudem, dass den Bildungseinrichtungen in der Projektkonzeption eher eine Rolle als Schnittstelle zukommt. Um den Bedürfnissen der einzelnen Schulformen und lokalen Standorte gerecht werden zu können, wäre eine stärkere Einbindung von der Ideenfindung bis hin zur Planung des Projektes notwendig.

Die Rentabilität der Kooperationszuschüsse wird hingegen wesentlich höher eingeschätzt, da es in diesem relativ kleinen Rahmen kaum zu nennenswerten Reibungsverlusten kommt. Außerdem geht man davon aus, dass mit dieser sehr niederschwelligen Form vermehrt auch eher weniger engagierte Bildungseinrichtungen angesprochen werden können, die zumeist einen hohen Förderbedarf haben. 


\section{Anhang}

\subsection{Erfolgsfaktoren und Wirkungen - Differenziert}

\section{Grafik 47: Erfolgsfaktoren - Differenziert Teil 1}

Welche dieser Aspekte haben sich im Rahmen der Projekte als besonders wichtig erwiesen, um Kinder/Jugendliche für Naturwissenschaft/Technik zu begeistern?

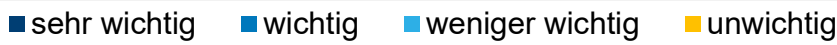

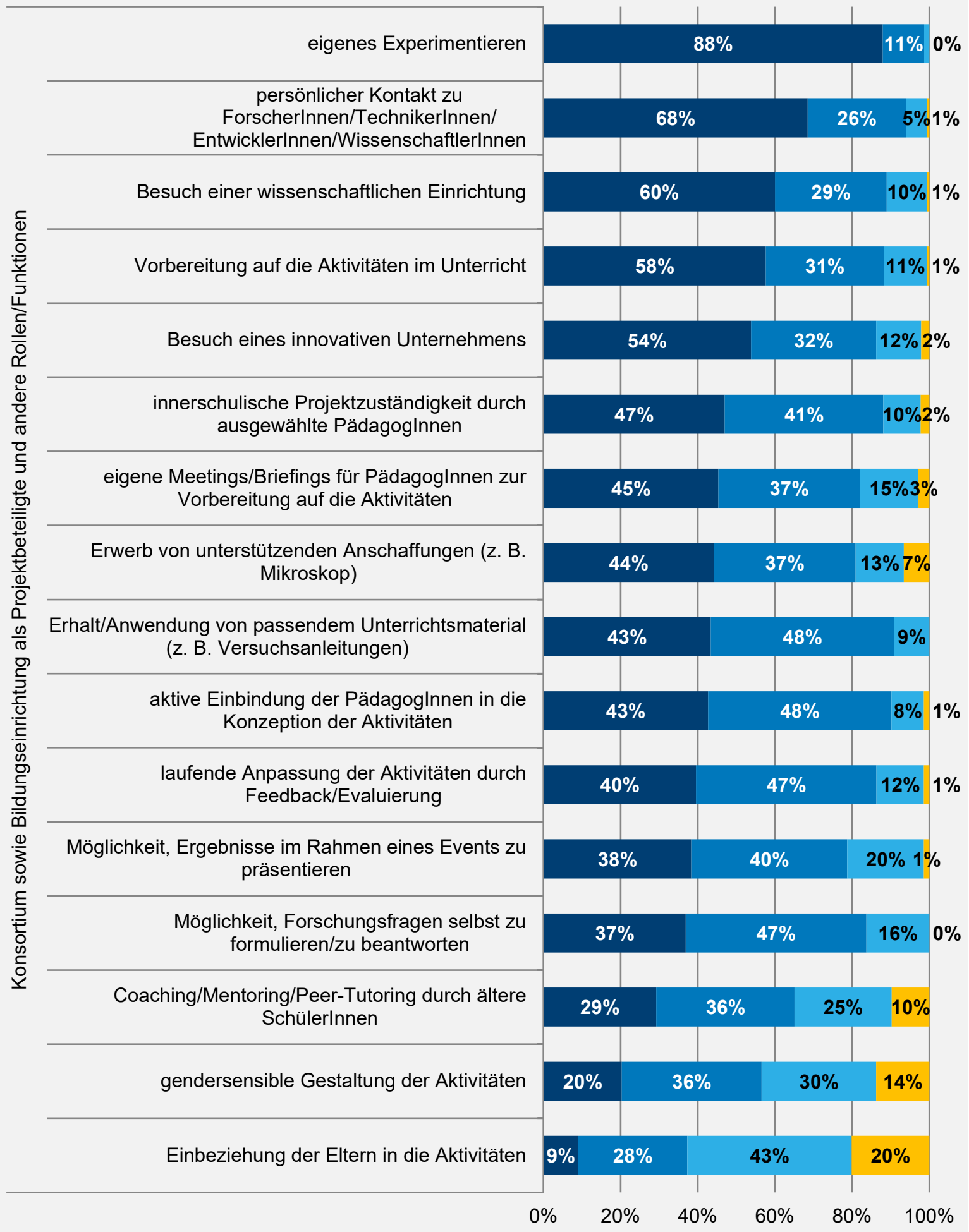

Quelle: ibw-Befragung 2017, n=123-149 


\section{Grafik 48: Erfolgsfaktoren - Differenziert Teil 2}

Welche dieser Aspekte haben sich im Rahmen der Projekte als besonders wichtig erwiesen, um Kinder/Jugendliche für Naturwissenschaft/Technik zu begeistern?

घehr wichtig $\quad$ wichtig $\quad$ weniger wichtig $\quad$ unwichtig

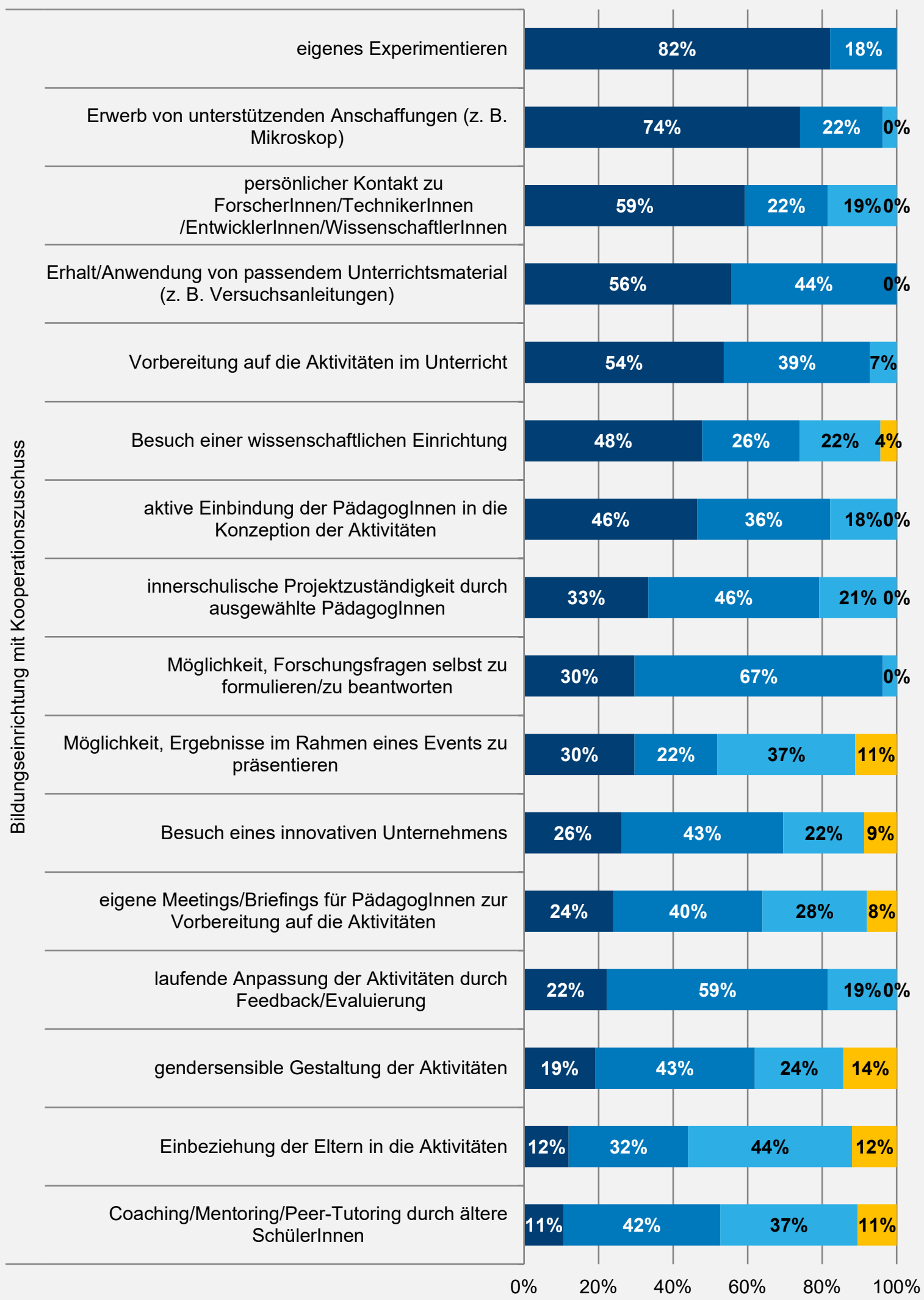




\section{Grafik 49: Wirkungen bei den Kindern und Jugendlichen - Differenziert}

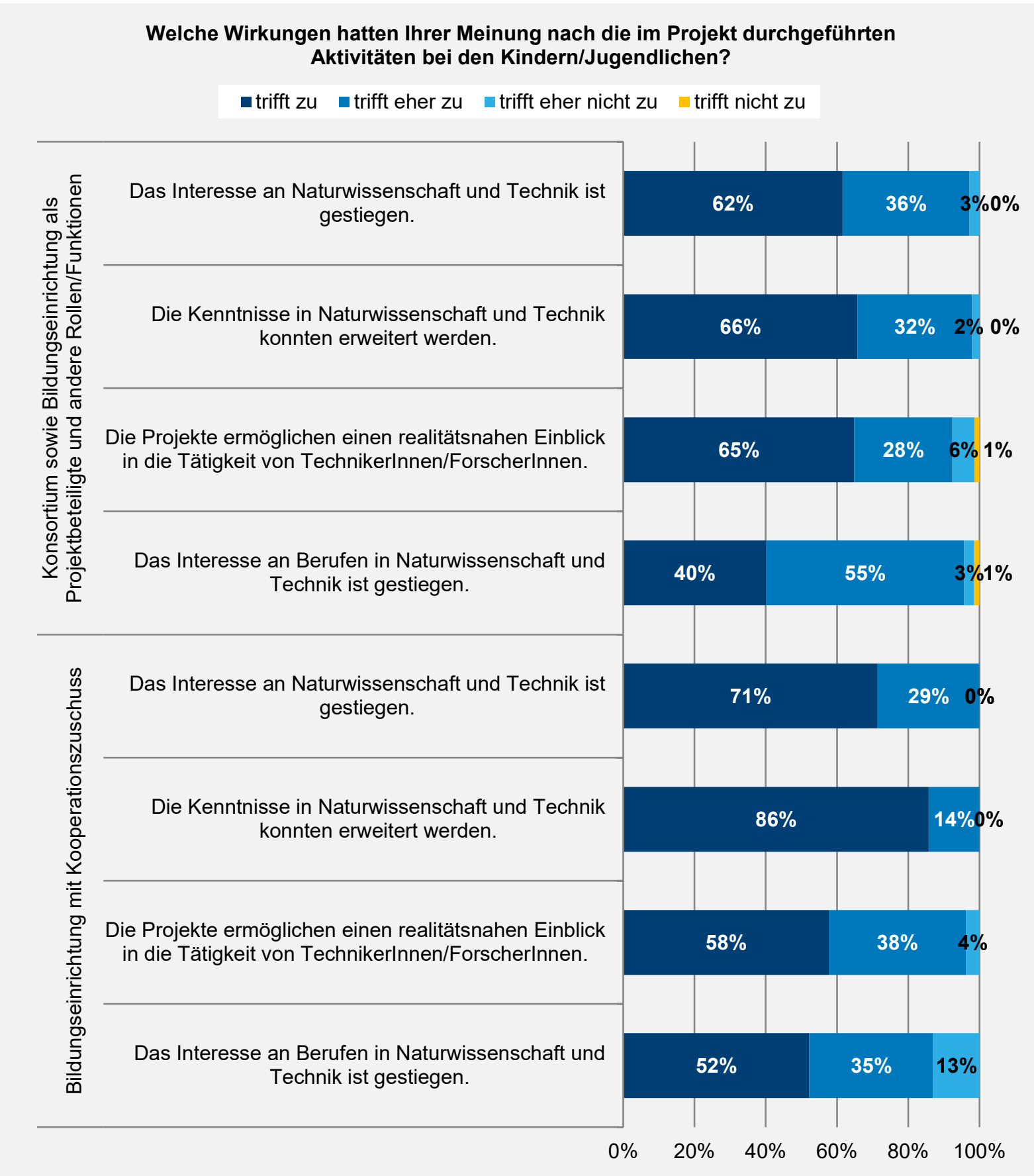

Quelle: ibw-Befragung 2017, n=137-143, n=23-28 


\section{Grafik 50: Wirkungen bei den PädagogInnen - Differenziert}

\section{Welche Wirkungen hatten Ihrer Meinung nach die im Projekt durchgeführten Aktivitäten bei den PädagogInnen?}

- trifft zu $\quad$ trifft eher zu $\quad$ trifft eher nicht zu $\quad$ trifft nicht zu

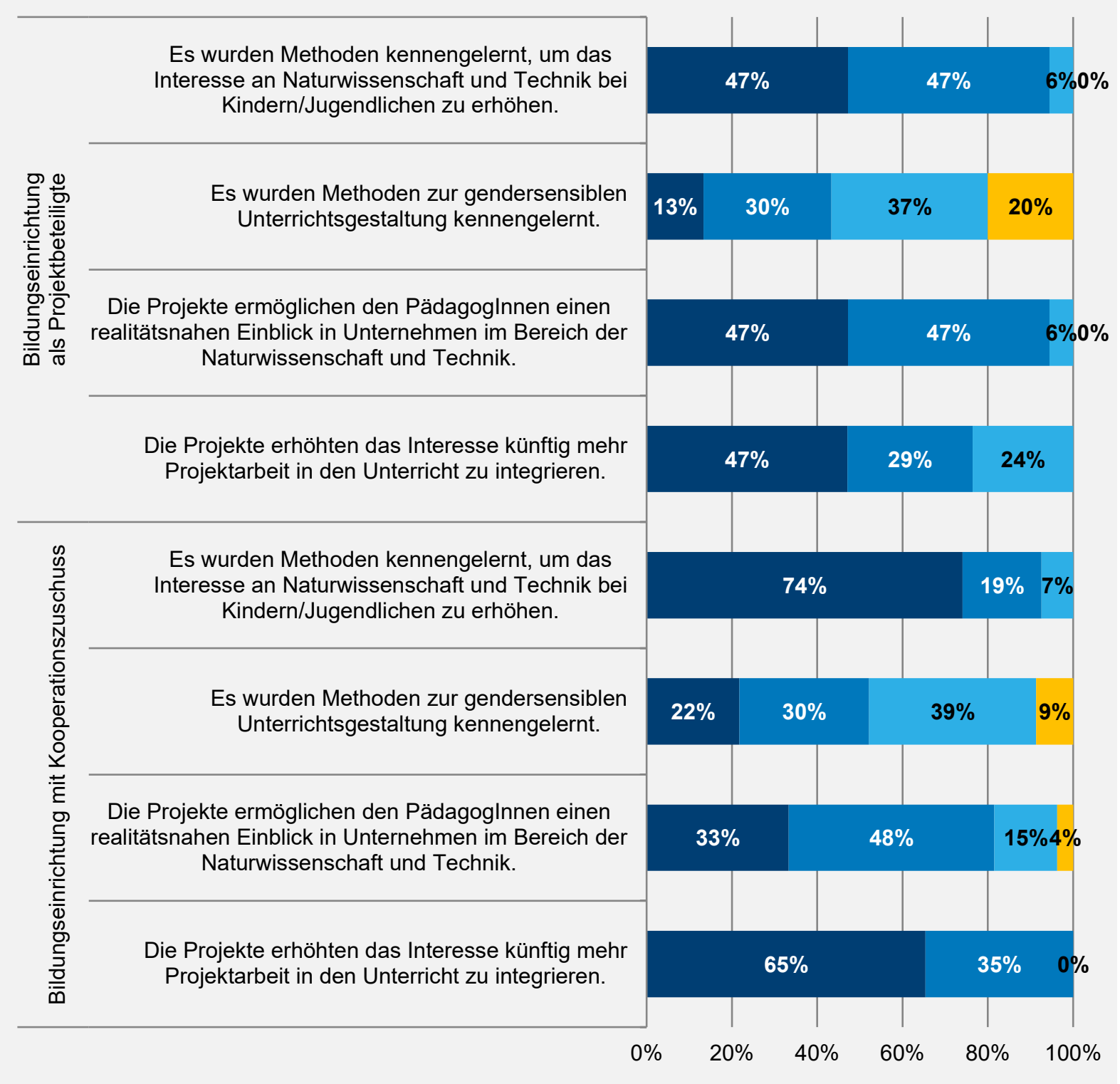

Quelle: ibw-Befragung 2017, n=30-36, n=23-27 


\section{Grafik 51: Wirkungen auf der Ebene der Schulen - Differenziert}

Welche Wirkungen hatten Ihrer Meinung nach die im Projekt durchgeführten Aktivitäten auf der Ebene der Schulen?

\section{Durch das Projekt ...}

- trifft zu $\quad$ trifft eher zu $\quad \square$ trifft eher nicht zu $\quad$ trifft nicht zu

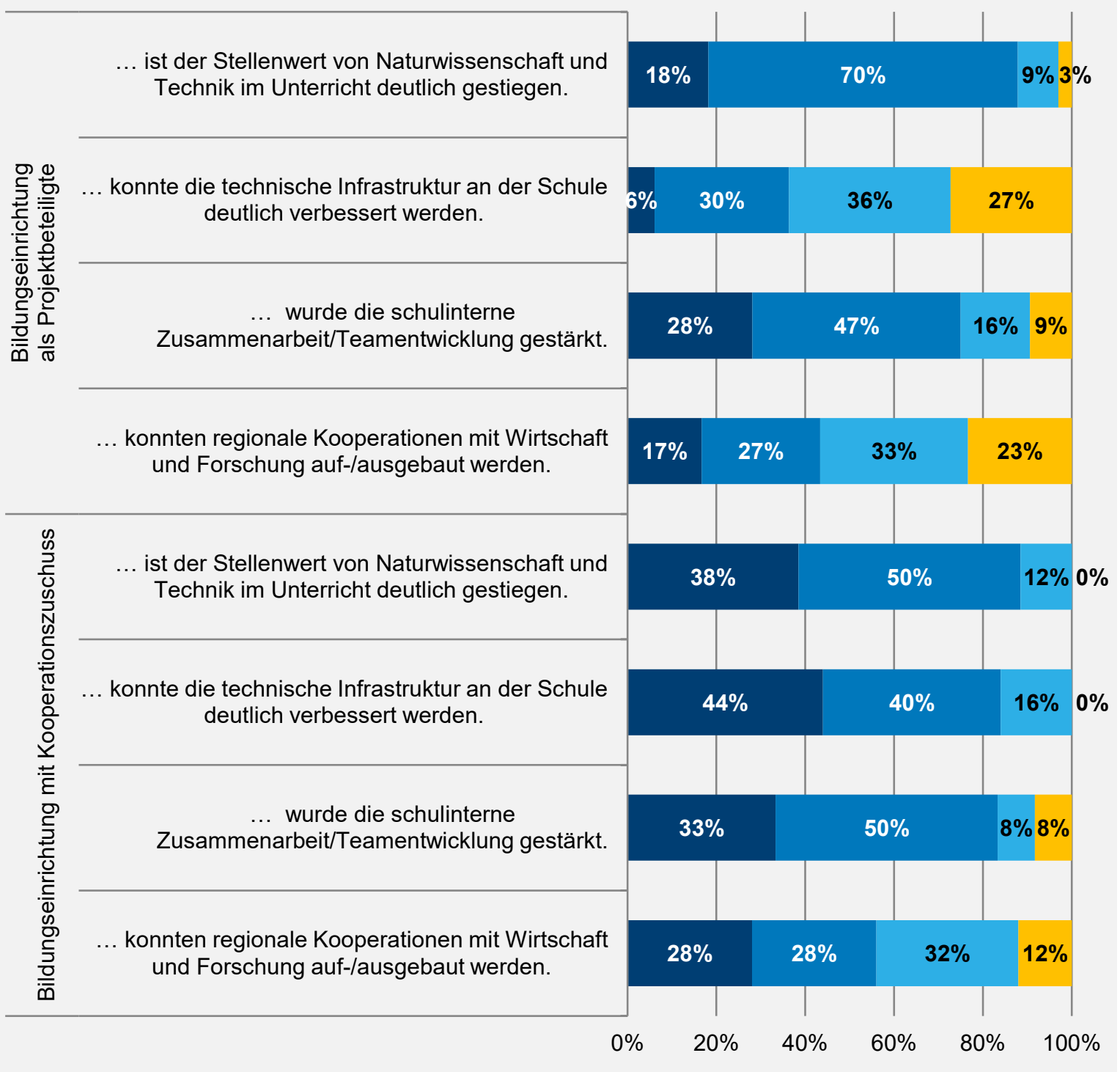

Quelle: ibw-Befragung 2017, n=27-33, n=24-26 


\subsection{Fragebogen Online-Befragung}

\section{Mehrere Projektteilnahmen}

1.1 Waren Sie an mehr als einem Projekt von „Talente regional“ bzw. "generation innovation Regionen“ beteiligt?

O ja $\rightarrow$ Frage 1.1.a

O nein $->$ Frage 2.1

1.1.a. An wie vielen Projekten von „Talente regional“ bzw. „generation innovation Regionen“ waren Sie bereits beteiligt?

Anzahl:

Falls der/die Befragte an mehreren Projekten von „Talente regional“ bzw. „generation innovation Regionen“ teilgenommen hat, kommt folgender Hinweis:

Sie haben angegeben, bereits an mehreren Projekten von „Talente regional“ bzw. „generation innovation Regionen" teilgenommen zu haben. Um eine möglichst differenzierte und genaue Zuordnung Ihrer Erfahrungen und Einschätzungen zu ermöglichen, bitten wir Sie sich im folgenden Fragebogen ausschließlich auf die letzte (aktuellste) Teilnahme zu beziehen.

\section{Ausschreibung, Rolle und Organisation}

2.1 Die Antworten im Rahmen dieser Befragung beziehen sich auf ein Projekt aus der:

O Ausschreibung "generation innovation Regionen (2010)

1. Ausschreibung Talente regional (2011)

O 2. Ausschreibung Talente regional (2012)

3. Ausschreibung Talente regional (2013)

O 4. Ausschreibung Talente regional (2014)

5. Ausschreibung Talente regional (2015)

6. Ausschreibung Talente regional (2016)

2.2 Zusätzlich können Sie auch den Titel des Projektes angeben:

2.3 Waren Sie in diesem Projekt für die Konsortialführung verantwortlich?

O ja $->$ Frage 2.3

nein -> Frage 2.4

2.3.a. Haben Sie zusätzlich zur Konsortialführung auch noch eine andere Rolle im Projekt übernommen?

O wissenschaftliche/r Partnerln

O Unternehmenspartnerln-> Frage 2.5.b und Frage 2.5.c

$O$ andere Rollen/Funktionen:

2.4 Welche Rolle haben Sie im Projekt übernommen?

O wissenschaftliche/r Partnerln

O Unternehmenspartnerln-> Frage 2.5.b und Frage 2.5.c

O (vor-)schulische Bildungseinrichtung als Projektbeteiligte -> Frage 2.5.a

O (vor-)schulische Bildungseinrichtung mit Kooperationszuschuss -> Frage 2.5.a

$O$ andere Rollen/Funktionen: 
2.5 Um welche Form von Organisation handelt es sich bei Ihrer Einrichtung?

O Außeruniversitäre Forschungseinrichtung

O Intermediär / Einrichtung des Technologietransfers (bspw. Gründungs- und Technologiezentrum, Unternehmenscluster, Science Center, Regionalmanagements etc.)

(vor-)schulische Bildungseinrichtung

Universität und Fachhochschule

O Unternehmen-> Frage 2.5.b und Frage 2.5.c

$O$ andere:

2.5.a. Welcher dieser Formen kann Ihre Organisation zugeordnet werden? (Mehrfachantworten möglich)

$\square$ Kindergarten

Volksschule

Polytechnische Schule

Neue Mittelschule/Hauptschule

Allgemeinbildende höhere Schule (Unterstufe)

Allgemeinbildende höhere Schule (Oberstufe)

$\checkmark$ Berufsbildende mittlere oder höhere Schule

$\square$ anderes:

2.5.b. In welcher Branche ist Ihr Unternehmen hauptsächlich tätig?

2.5.c. Bitte schätzen Sie den Anteil der Beschäftigten in Ihrem Unternehmen, die dem Berufsfeld der „MINTBerufe" zugeordnet werden können.

Es handelt sich dabei um Tätigkeiten, für deren Ausübung ein hoher Anteil an Kenntnissen in den Bereichen Mathematik, Informatik, Naturwissenschaften und/oder Technik erforderlich ist. Dabei wird auch das Bauen und Instandhalten technischer Anlagen und Geräte als zentraler Bestandteil gezählt.

\% der Beschäftigen in „MINT-Berufen“ 


\section{Projektidee und Kontaktaufnahme}

3.1 Wer gab den entscheidenden Impuls (Projektidee) für die Beteiligung an der Ausschreibung? (Mehrfachantworten möglich)

meine Organisation (als Konsortialführung)

wissenschaftliche/r Partnerln

$\square$ Unternehmenspartnerln

$\square$ (vor-)schulische Bildungseinrichtung

$\square$ andere:

3.2 Wie kam der Kontakt zum Projektkonsortium zustande? Es handelt sich um ...

\begin{tabular}{|c|c|c|c|c|c|c|c|c|}
\hline & \multicolumn{4}{|c|}{ wissenschaftliche/r Partnerln } & \multicolumn{4}{|c|}{ Unternehmenspartnerln } \\
\hline & $\begin{array}{c}\text { trifft } \\
\mathrm{zu}\end{array}$ & $\begin{array}{l}\text { trifft } \\
\text { eher } \\
\mathrm{zu}\end{array}$ & $\begin{array}{l}\text { trifft } \\
\text { eher } \\
\text { nicht } \\
\text { zu }\end{array}$ & $\begin{array}{c}\text { trifft } \\
\text { nicht } \\
\mathrm{zu}\end{array}$ & $\begin{array}{c}\text { trifft } \\
\mathrm{zu}\end{array}$ & $\begin{array}{l}\text { trifft } \\
\text { eher } \\
\mathrm{zu}\end{array}$ & $\begin{array}{l}\text { trifft } \\
\text { eher } \\
\text { nicht } \\
\text { zu }\end{array}$ & $\begin{array}{c}\text { trifft } \\
\text { nicht } \\
\text { zu }\end{array}$ \\
\hline $\begin{array}{l}\text {... „neue“ Kontakte, die erst im Rahmen } \\
\text { des Projektes entstanden sind }\end{array}$ & $\mathrm{O}$ & $\mathrm{O}$ & $\mathrm{O}$ & O & $\mathrm{O}$ & $\mathrm{O}$ & $\mathrm{O}$ & $\mathrm{O}$ \\
\hline $\begin{array}{l}\text {... bestehende Kontakte/Netzwerke - ohne } \\
\text { bisherige Projektzusammenarbeit }\end{array}$ & $\mathrm{O}$ & $\mathrm{O}$ & $\mathrm{O}$ & $\mathrm{O}$ & $\mathrm{O}$ & $\mathrm{O}$ & $\mathrm{O}$ & $\mathrm{O}$ \\
\hline $\begin{array}{l}\text {... bestehende Kontakte/Netzwerke - mit } \\
\text { Projektzusammenarbeit in der Vergangen- } \\
\text { heit }\end{array}$ & $\mathrm{O}$ & $\mathrm{O}$ & $\mathrm{O}$ & $\mathrm{O}$ & $\mathrm{O}$ & $\mathrm{O}$ & $\mathrm{O}$ & $\mathrm{O}$ \\
\hline
\end{tabular}

3.3 Wie kam der Kontakt zu den (vor-)schulischen Bildungseinrichtungen zustande? Es handelt sich um ...

\begin{tabular}{|c|c|c|c|c|c|c|c|c|}
\hline & \multicolumn{4}{|c|}{$\begin{array}{l}\text { (vor-)schulische Bildungseinrich- } \\
\text { tungen als Projektbeteiligte }\end{array}$} & \multicolumn{4}{|c|}{$\begin{array}{c}\text { (vor-)schulische Bildungseinrich- } \\
\text { tungen mit Kooperationszu- } \\
\text { schuss }\end{array}$} \\
\hline & $\begin{array}{c}\text { trifft } \\
\mathrm{zu}\end{array}$ & $\begin{array}{l}\text { trifft } \\
\text { eher } \\
\mathrm{zu}\end{array}$ & $\begin{array}{c}\text { trifft } \\
\text { eher } \\
\text { nicht } \\
\text { zu }\end{array}$ & $\begin{array}{l}\text { trifft } \\
\text { nicht } \\
\text { zu }\end{array}$ & $\begin{array}{c}\text { trifft } \\
\mathrm{zu}\end{array}$ & $\begin{array}{l}\text { trifft } \\
\text { eher } \\
\mathrm{zu}\end{array}$ & $\begin{array}{c}\text { trifft } \\
\text { eher } \\
\text { nicht } \\
\text { zu }\end{array}$ & $\begin{array}{c}\text { trifft } \\
\text { nicht } \\
\mathrm{zu}\end{array}$ \\
\hline $\begin{array}{l}\text {... „neue“ Kontakte, die erst im Rahmen } \\
\text { des Projektes entstanden sind }\end{array}$ & 0 & 0 & 0 & 0 & 0 & 0 & 0 & 0 \\
\hline $\begin{array}{l}\text {... bestehende Kontakte/Netzwerke - ohne } \\
\text { bisherige Projektzusammenarbeit }\end{array}$ & 0 & 0 & 0 & 0 & 0 & 0 & 0 & 0 \\
\hline $\begin{array}{l}\text {... bestehende Kontakte/Netzwerke - mit } \\
\text { Projektzusammenarbeit in der Vergangen- } \\
\text { heit }\end{array}$ & 0 & 0 & 0 & 0 & 0 & 0 & 0 & 0 \\
\hline
\end{tabular}


Abschnitt 4 richtet sich an alle Projektbeteiligten außer Bildungseinrichtungen mit Kooperationszuschuss.

\section{Projektabwicklung und -gestaltung}

4.1 Wie zufrieden sind Sie mit den verschiedenen Vorgaben der Ausschreibung und der Projektabwicklung?

\begin{tabular}{|l|c|c|c|c|}
\hline & $\begin{array}{c}\text { sehr } \\
\text { zufrieden }\end{array}$ & zufrieden & $\begin{array}{c}\text { weniger } \\
\text { zufrieden }\end{array}$ & $\begin{array}{c}\text { nicht } \\
\text { zufrieden }\end{array}$ \\
\hline Laufzeit der Projekte & O & O & 0 \\
\hline Förderhöhe/Projektbudget & 0 & 0 & 0 & 0 \\
\hline Konzeption der Kooperationszuschüsse & 0 & 0 & 0 & 0 \\
\hline Zeitpunkt der Ausschreibung & 0 & 0 & 0 & 0 \\
\hline Darstellung der Förderkriterien & 0 & 0 & 0 & 0 \\
\hline formaler Ablauf der Antragstellung & 0 & 0 & 0 & 0 \\
\hline Dauer bis zur Förderentscheidung & 0 & 0 & 0 & 0 \\
\hline Zeitpunkt (Deadline) der Förderentscheidung & 0 & 0 & 0 & 0 \\
\hline Berichtslegung & 0 & 0 & 0 & 0 \\
\hline Abrechnungsmodalitäten & 0 & 0 & 0 & 0 \\
\hline Kontakt bzw. Betreuung durch die FFG & 0 & 0 & 0 \\
\hline
\end{tabular}

4.2 Wie zufrieden sind Sie mit den einzelnen Aspekten innerhalb des Projektkonsortiums?

\begin{tabular}{|l|c|c|c|c|}
\hline & $\begin{array}{c}\text { sehr } \\
\text { zufrieden }\end{array}$ & zufrieden & $\begin{array}{c}\text { weniger } \\
\text { zufrieden }\end{array}$ & $\begin{array}{c}\text { nicht } \\
\text { zufrieden }\end{array}$ \\
\hline $\begin{array}{l}\text { Projektmanagement (Konsortialführung) } \\
->(\text { Frage 2.3 f JA) }\end{array}$ & 0 & 0 & 0 \\
\hline Zeitmanagement (Terminfindung) & 0 & 0 & 0 & 0 \\
\hline Kommunikation (inhaltliche Abstimmung) & 0 & 0 & 0 & 0 \\
\hline Aufteilung der Verantwortlichkeiten & 0 & 0 & 0 & 0 \\
\hline $\begin{array}{l}\text { Aufteilung der Projektadministration (Berichte und } \\
\text { Abrechnungen) }\end{array}$ & 0 & 0 & 0 & 0 \\
\hline Engagement der Projektbeteiligten & 0 & 0 & 0 & 0 \\
\hline
\end{tabular}


Abschnitt 5 richtet sich an alle Projektbeteiligten außer Bildungseinrichtungen mit Kooperationszuschuss.

\section{Ausschreibungsschwerpunkte}

5.1 Sind Sie der Meinung, dass „Talente regional“ bzw. „generation innovation Regionen“ dafür geeignet ist, die folgenden Ziele/Anforderungen (Ausschreibungsschwerpunkte) umzusetzen? Talente regional ist grundsätzlich dafür geeignet ...

\begin{tabular}{|c|c|c|c|c|}
\hline & trifft zu & $\begin{array}{c}\text { trifft } \\
\text { eher zu }\end{array}$ & $\begin{array}{l}\text { trifft } \\
\text { eher } \\
\text { nicht zu }\end{array}$ & $\begin{array}{c}\text { trifft } \\
\text { nicht zu }\end{array}$ \\
\hline $\begin{array}{l}\ldots \text { das Interesse von Kinder und Jugendlichen an Forschung, } \\
\text { Technologie und Innovation zu steigern. }\end{array}$ & O & 0 & $\mathrm{O}$ & O \\
\hline $\begin{array}{l}\text {... den Bezug zu Naturwissenschaft und Technik von Kindern } \\
\text { und Jugendlichen durch die aktive Einbindung in die Projekte zu } \\
\text { vertiefen. }\end{array}$ & 0 & 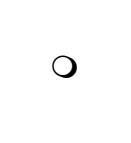 & O & $\mathrm{O}$ \\
\hline $\begin{array}{l}\text {... Gender-Aspekte bzw. Geschlechterausgewogenheit (bspw. } \\
\text { durch gezieltes Ansprechen von Mädchen und jungen Frauen) } \\
\text { zu berücksichtigen. }\end{array}$ & 0 & $\mathrm{O}$ & O & $\mathrm{O}$ \\
\hline $\begin{array}{l}\text {... Kinder und Jugendliche unabhängig ihrer sozialen oder } \\
\text { geografischen Herkunft (bspw. mit Migrationshintergrund) aktiv } \\
\text { einzubinden. }\end{array}$ & 0 & O & O & $\mathrm{O}$ \\
\hline $\begin{array}{l}\text {... die Vernetzung von (vor-)schulischen Bildungseinrichtungen } \\
\text { und PartnerInnen aus Wirtschaft und Forschung zu stärken. }\end{array}$ & O & $\mathrm{O}$ & O & $\mathrm{O}$ \\
\hline
\end{tabular}

5.2 Wie anspruchsvoll war es, all diese (oben genannten) Ziele/Anforderungen im Rahmen des Projektes zu berücksichtigen?
O sehr
O eher
anspruchsvoll
anspruchsvoll
O weniger
anspruchsvoll
gar nicht anspruchsvoll

5.3 Wie bzw. in welcher Form konnten diese (oben genannten) Ziele/Anforderungen im Rahmen des Projektes miteinander in Einklang gebracht werden? 
Abschnitt 6 richtet sich an alle Projektbeteiligten inklusive der Bildungseinrichtungen mit Kooperationszuschuss.

\section{Erfolgsfaktoren}

6.1 Welche dieser Aspekte haben sich im Rahmen der Projekte als besonders wichtig erwiesen, um Kinder/Jugendliche für Naturwissenschaft/Technik zu begeistern?

\begin{tabular}{|c|c|c|c|c|}
\hline & $\begin{array}{l}\text { sehr } \\
\text { wichtig }\end{array}$ & wichtig & $\begin{array}{l}\text { weniger } \\
\text { wichtig }\end{array}$ & $\begin{array}{c}\text { nicht } \\
\text { wichtig }\end{array}$ \\
\hline Vorbereitung auf die Aktivitäten im Unterricht & $\mathrm{O}$ & $\mathrm{O}$ & $\mathrm{O}$ & $\mathrm{O}$ \\
\hline $\begin{array}{l}\text { Erhalt/Anwendung von passendem Unterrichtsmaterial } \\
\text { (z. B. Versuchsanleitungen) }\end{array}$ & $\mathrm{O}$ & $\mathrm{O}$ & $\mathrm{O}$ & $\mathrm{O}$ \\
\hline $\begin{array}{l}\text { Erwerb von unterstützenden Anschaffungen (z. B. } \\
\text { Mikroskop) }\end{array}$ & $\mathrm{O}$ & O & $\mathrm{O}$ & $\mathrm{O}$ \\
\hline $\begin{array}{l}\text { Möglichkeit, Forschungsfragen selbst zu formulieren/zu } \\
\text { beantworten }\end{array}$ & $\mathrm{O}$ & $\mathrm{O}$ & $\mathrm{O}$ & $\mathrm{O}$ \\
\hline eigenes Experimentieren & $\mathrm{O}$ & $\mathrm{O}$ & $\mathrm{O}$ & $\mathrm{O}$ \\
\hline $\begin{array}{l}\text { Möglichkeit, Ergebnisse im Rahmen eines Events zu } \\
\text { präsentieren }\end{array}$ & $\mathrm{O}$ & O & $\mathrm{O}$ & $\mathrm{O}$ \\
\hline $\begin{array}{l}\text { Coaching/Mentoring/Peer-Tutoring durch ältere Schüle- } \\
\text { rlnnen }\end{array}$ & O & O & O & $\mathrm{O}$ \\
\hline Besuch eines innovativen Unternehmens & $\mathrm{O}$ & $\mathrm{O}$ & $\mathrm{O}$ & $\mathrm{O}$ \\
\hline Besuch einer wissenschaftlichen Einrichtung & $\mathrm{O}$ & O & $\mathrm{O}$ & $\mathrm{O}$ \\
\hline $\begin{array}{l}\text { persönlicher Kontakt zu ForscherInnen/TechnikerInnen/ } \\
\text { EntwicklerInnen/WissenschaftlerInnen }\end{array}$ & $\mathrm{O}$ & $\mathrm{O}$ & $\mathrm{O}$ & $\mathrm{O}$ \\
\hline Einbeziehung der Eltern in die Aktivitäten & $\mathrm{O}$ & $\mathrm{O}$ & $\mathrm{O}$ & $\mathrm{O}$ \\
\hline gendersensible Gestaltung der Aktivitäten & $\mathrm{O}$ & $\mathrm{O}$ & $\mathrm{O}$ & $\mathrm{O}$ \\
\hline $\begin{array}{l}\text { eigene Meetings/Briefings für PädagogInnen zur } \\
\text { Vorbereitung auf die Aktivitäten }\end{array}$ & $\mathrm{O}$ & $\mathrm{O}$ & $\mathrm{O}$ & $\mathrm{O}$ \\
\hline $\begin{array}{l}\text { aktive Einbindung der PädagogInnen in die Konzeption } \\
\text { der Aktivitäten }\end{array}$ & $\mathrm{O}$ & $\mathrm{O}$ & $\mathrm{O}$ & $\mathrm{O}$ \\
\hline $\begin{array}{l}\text { innerschulische Projektzuständigkeit durch ausgewählte } \\
\text { PädagogInnen }\end{array}$ & O & $\mathrm{O}$ & $\mathrm{O}$ & $\mathrm{O}$ \\
\hline $\begin{array}{l}\text { laufende Anpassung der Aktivitäten durch Feed- } \\
\text { back/Evaluierung }\end{array}$ & O & O & $\mathrm{O}$ & $\mathrm{O}$ \\
\hline
\end{tabular}

6.2 Andere Aspekte, die wichtig sind, um Kinder/Jugendliche für Naturwissenschaft/ Technik zu begeistern? 


\section{Wirkungen der im Projekt durchgeführten Aktivitäten}

Frage 7.1 richtet sich an alle Projektbeteiligten inklusive der Bildungseinrichtungen mit Kooperationszuschuss.

7.1 Welche Wirkungen hatten Ihrer Meinung nach die im Projekt durchgeführten Aktivitäten bei den Kindern/Jugendlichen?

\begin{tabular}{|l|c|c|c|c|}
\hline & trifft zu & $\begin{array}{c}\text { trift eher } \\
\text { zu }\end{array}$ & $\begin{array}{c}\text { trifft eher } \\
\text { nicht zu }\end{array}$ & $\begin{array}{c}\text { trifft nicht } \\
\text { zu }\end{array}$ \\
\hline $\begin{array}{l}\text { Das Interesse an Naturwissenschaft und Technik ist } \\
\text { gestiegen. }\end{array}$ & 0 & 0 & 0 \\
\hline $\begin{array}{l}\text { Die Kenntnisse in Naturwissenschaft und Technik } \\
\text { konnten erweitert werden. }\end{array}$ & 0 & 0 & 0 & 0 \\
\hline $\begin{array}{l}\text { Die Projekte ermöglichen einen realitätsnahen Einblick in } \\
\text { die Tätigkeit von TechnikerInnen/Forscherlnnen. }\end{array}$ & 0 & 0 & 0 & 0 \\
\hline $\begin{array}{l}\text { Das Interesse an Berufen in Naturwissenschaft und } \\
\text { Technik ist gestiegen. }\end{array}$ & 0 & 0 & 0 & 0 \\
\hline
\end{tabular}

Frage 7.2 richtet sich nur an Bildungseinrichtungen

7.2 Welche Wirkungen hatten Ihrer Meinung nach die im Projekt durchgeführten Aktivitäten bei den $\underline{\text { Päda- }}$ goglnnen?

\begin{tabular}{|l|c|c|c|c|}
\hline & trifft zu & $\begin{array}{c}\text { trifft eher } \\
\text { zu }\end{array}$ & $\begin{array}{c}\text { trifft eher } \\
\text { nicht zu }\end{array}$ & $\begin{array}{c}\text { trifft nicht } \\
\text { zu }\end{array}$ \\
\hline $\begin{array}{l}\text { Es wurden Methoden kennengelernt, um das Interesse } \\
\text { a Naturwissenschaft und Technik bei Kin- } \\
\text { dern/Jugendlichen zu erhöhen. }\end{array}$ & 0 & 0 & 0 \\
\hline $\begin{array}{l}\text { Es wurden Methoden zur gendersensiblen Unterrichts- } \\
\text { gestaltung kennengelernt. }\end{array}$ & 0 & 0 & 0 & 0 \\
\hline $\begin{array}{l}\text { Die Projekte ermöglichen den PädagogInnen einen } \\
\text { realitätsnahen Einblick in Unternehmen im Bereich der } \\
\text { Naturwissenschaft und Technik. }\end{array}$ & 0 & 0 & 0 & 0 \\
\hline $\begin{array}{l}\text { Die Projekte erhöhten das Interesse künftig mehr } \\
\text { Projektarbeit in den Unterricht zu integrieren. }\end{array}$ & 0 & 0 & 0 & 0 \\
\hline
\end{tabular}


Frage 7.3 richtet sich nur an Bildungseinrichtungen.

7.3 Welche Wirkungen hatten Ihrer Meinung nach die im Projekt durchgeführten Aktivitäten auf der Ebene der Schulen? Durch das Projekt ...

\begin{tabular}{|l|c|c|c|c|}
\hline & trifft zu & $\begin{array}{c}\text { trifft eher } \\
\text { zu }\end{array}$ & $\begin{array}{c}\text { trifft eher } \\
\text { nicht zu }\end{array}$ & $\begin{array}{c}\text { trifft nicht } \\
\text { zu }\end{array}$ \\
\hline $\begin{array}{l}\ldots \text { ist der Stellenwert von Naturwissenschaft und Technik } \\
\text { im Unterricht deutlich gestiegen. }\end{array}$ & 0 & 0 & 0 \\
\hline $\begin{array}{l}\ldots \text { konnte die technische Infrastruktur an der Schule } \\
\text { deutlich verbessert werden. }\end{array}$ & 0 & 0 & 0 & 0 \\
\hline $\begin{array}{l}\text {... wurde die schulinterne Zusammenarbeit/ } \\
\text { Teamentwicklung gestärkt. }\end{array}$ & 0 & 0 & 0 & 0 \\
\hline $\begin{array}{l}\text { F. konnten regionale Kooperationen mit Wirtschaft und } \\
\text { Forschung auf-/ausgebaut werden. }\end{array}$ & 0 & 0 & 0 & 0 \\
\hline
\end{tabular}

Abschnitt 8 richtet sich an alle Projektbeteiligten inklusive Bildungseinrichtungen mit Kooperationszuschuss, sofern die Teilnahme nicht im Rahmen der Ausschreibung „generation innovation Regionen“ (2010) war.

\section{Wirkung der Kooperationszuschüsse}

8.1 Welche Wirkungen haben Ihrer Meinung nach die vergebenen Kooperationszuschüsse?

\begin{tabular}{|c|c|c|c|c|}
\hline & trifft zu & $\begin{array}{l}\text { trifft eher } \\
\mathrm{zu}\end{array}$ & $\begin{array}{l}\text { trifft eher } \\
\text { nicht zu }\end{array}$ & $\begin{array}{l}\text { trifft nicht } \\
\mathrm{zu}\end{array}$ \\
\hline $\begin{array}{l}\text { Sie ermöglichen die Förderung von Aktivitäten mit einem } \\
\text { geringen formalen Aufwand. }\end{array}$ & 0 & 0 & 0 & $\mathrm{O}$ \\
\hline $\begin{array}{l}\text { Sie erweitern die Vielfalt der Projektteilnehmerlnnen } \\
\text { entscheidend. }\end{array}$ & O & 0 & 0 & O \\
\hline $\begin{array}{l}\text { Sie vergrößern die Anzahl der angesprochenen Kinder } \\
\text { und Jugendlichen im hohen Maße. }\end{array}$ & O & O & O & O \\
\hline $\begin{array}{l}\text { Sie tragen wesentlich zur Bekanntheit von „Talente } \\
\text { regional“ und deren Projekten in der Öffentlichkeit bei. }\end{array}$ & O & O & O & O \\
\hline $\begin{array}{l}\text { Sie sind eine gute Basis für die breitere Vernetzung von } \\
\text { (vor-)schulischen Bildungseinrichtungen mit Partnerln- } \\
\text { nen aus Wirtschaft und Forschung. }\end{array}$ & 0 & 0 & O & O \\
\hline $\begin{array}{l}\text { Sie begünstigen das Interesse, sich vermehrt mit der } \\
\text { Inanspruchnahme von Förderungen auseinanderzuset- } \\
\text { zen. }\end{array}$ & $\mathrm{O}$ & O & O & $\mathrm{O}$ \\
\hline
\end{tabular}


Abschnitt 9 richtet sich an alle Projektbeteiligten inklusive Bildungseinrichtungen mit Kooperationszuschuss, sofern die Teilnahme nicht im Rahmen der 5. und 6. Ausschreibung (2015-2016) war.

\section{Nachhaltigkeit der Kooperationen}

9.1 Sind nach Projektabschluss nachhaltige Kooperationen mit einzelnen Organisationen entstanden?

○ ja $\rightarrow>$ Frage 9.2

O nein -> Frage 9.4

9.2 Wie intensiv ist diese Zusammenarbeit?

\begin{tabular}{|l|c|c|c|c|}
\hline & stark & mittelmäßig & schwach & keine \\
\hline Außeruniversitäre Forschungseinrichtungen & 0 & 0 & 0 \\
\hline $\begin{array}{l}\text { Intermediäre/Einrichtungen des Technologietrans- } \\
\text { fers (bspw. Gründungs- und Technologiezentren, } \\
\text { Unternehmenscluster, Science Center, Regionalma- } \\
\text { nagements etc.) }\end{array}$ & O & 0 & 0 & 0 \\
\hline $\begin{array}{l}\text { (vor-)schulische Bildungseinrichtungen } \\
\text { Universitäten und Fachhochschulen }\end{array}$ & 0 & 0 & 0 & 0 \\
\hline Unternehmen & 0 & 0 & 0 & 0 \\
\hline
\end{tabular}

9.3 Um welche Form/en der Zusammenarbeit handelt es sich? Die Zusammenarbeit ...

\begin{tabular}{|l|c|c|c|c|}
\hline & trifft zu & trifft eher zu & $\begin{array}{c}\text { trifft eher } \\
\text { nicht zu }\end{array}$ & $\begin{array}{c}\text { trifft } \\
\text { nicht zu }\end{array}$ \\
\hline$\ldots$ schließt direkt an das Projekt an. & 0 & 0 & 0 & 0 \\
\hline$\ldots$ hat einen anderen thematischen Fokus. & 0 & 0 & 0 & 0 \\
\hline$\ldots$ hat eine andere Zielgruppe. & 0 & 0 & 0 & 0 \\
\hline $\begin{array}{l}\ldots \text { entwickelt das pädagogisch/didaktische Konzept } \\
\text { weiter. }\end{array}$ & 0 & 0 & 0 & 0 \\
\hline
\end{tabular}

9.4 Welche Gründe gibt es Ihrer Meinung nach, dass es nach Projektabschluss zu keiner nachhaltigen Zusammenarbeit mit einzelnen Organisationen gekommen ist? 
Abschnitt 10 richtet sich an alle Projektbeteiligten inklusive Bildungseinrichtungen mit Kooperationszuschuss.

\section{Vorerfahrungen}

10.1 Wie bzw. von wem haben Sie zum ersten Mal von "Talente regional“ bzw. „generation innovation Regionen" erfahren? (Mehrfachantworten möglich)

$\square$ Medienberichte

$\square$ eigene Recherche

Kontakt mit BMVIT/FFG

Informationen/Veranstaltungen des BMVIT/FFG

$\square$ Konsortialführung

Wissenschaftliche/r Partnerln

Unternehmenspartnerln

$\square$ (vor-)schulische Bildungseinrichtung

$\square$ andere Kontakte/Kanäle:.

10.2 Haben Sie im Förderschwerpunkt „Talente“ des BMVIT über Talente regional hinausgehend auch an anderen Förderformaten (bspw. Praktika für SchülerInnen, FEMtech Karriere, etc.) teilgenommen?

O ja $\rightarrow$ Frage 10.3

O nein -> Frage 10.4

10.3 Um welches Förderformat handelt es sich genau? (Mehrfachantworten möglich)

$\square$ Praktika für Schülerinnen und Schüler

$\square$ FEMtech Forschungsprojekte

FEMtech Karriere/FEMtech Karriere-Check für KMU

$\square$ FEMtech Praktika für Studentinnen

FEMtech Dissertationen

$\square$ Die österreichische Jobbörse für Forschung, Entwicklung und Innovation bzw. Praktikabörse

10.4 Haben Sie auch schon an anderen Initiativen zur Förderung des Interesses des Nachwuchses an Naturwissenschaft und Technik (bspw. Kooperationen, Exkursionen, Sparkling Science etc.) teilgenommen?

O ja $->$ Frage 10.4.a

O nein -> Frage 11.1

10.4.a. Wenn ja, um welche Initiativen handelte es sich?

Bitte um stichwortartige Angaben: 
Abschnitt 11 richtet sich an alle Projektbeteiligten inklusive Bildungseinrichtungen mit Kooperationszuschuss.

\section{Abschlussfragen}

11.1 Bitte teilen Sie uns Ihre Einschätzungen/Erfahrungen mit, die Sie im Rahmen der Umsetzung des Projekts gemacht haben.

Mit welchen Herausforderungen waren Sie in den Projekten konfrontiert?

Haben Sie konkrete Vorschläge für Verbesserungen?

Welche positiven Erfahrungen haben Sie im Rahmen der Projekte gemacht?

Frage 11.2 richtet sich an alle Projektbeteiligten inklusive Bildungseinrichtungen mit Kooperationszuschuss, die mehrere Projektbeteiligungen aufweisen.

Sie haben zu Beginn des Fragebogens angegeben, bereits an mehreren Projekten von „Talente regional“ bzw. "generation innovation Regionen“ teilgenommen zu haben.

11.2 Gibt es zwischen den verschiedenen Ausschreibungen, Projekten bzw. Projektkonsortien, an denen Sie teilgenommen haben, erwähnenswerte Unterschiede? Bitte teilen Sie uns Ihre Einschätzungen/Erfahrungen mit.

11.3 Im Rahmen der Befragung sind zur Vertiefung bestimmter Schwerpunkte persönliche Interviews (telefonisch) geplant. Dürfen wir Sie diesbezüglich kontaktieren? Falls ja, würden wir uns freuen, wenn Sie uns Ihre Kontaktdaten (E-Mail-Adresse oder Telefonnummer) bekannt geben:

Vielen herzlichen Dank für Ihre Teilnahme! 


\subsection{Leitfaden Interviews}

\section{Persönliche Daten}

\begin{tabular}{l}
\hline Name \\
\hline Funktion/Beruf \\
\hline Institution \\
\hline Projektbeteiligung \\
\hline Kontaktdaten \\
\hline Anonymität erwünscht? \\
\hline
\end{tabular}

\section{Schwerpunkt 1 (Bildungseinrichtungen)}

1. Wie würden Sie den Impact der durchgeführten Aktivitäten beschreiben? Impact in Bezug auf...

$>$... Didaktik/Lehrplan/Unterrichtsmaterialien

$>$...PädagogInnen/Teambuilding

$>$... „externe“ Kontakte/Netzwerke/Kooperationen

$>$....Projektbeteiligung als treibende Kraft der Schulentwicklung

2. Woran erkennen Sie die verschiedenen Wirkungen/Effekte?

$>$ Beobachtungen/direktes Feedback

$>$ Indikatoren/Instrumente/Evaluierungen

3. Gab/Gibt es auch negative Auswirkungen auf die eigene Bildungseinrichtung, die bedacht werden sollten?

$>$ Beispiele für Irritationen/Störfaktoren

$>$ Trade-off Konstellationen/Prioritäten

4. Reflexionen zum komplexen Projektdesign?

$>$ Vereinbarkeit der Zielsetzungen

$>$ Umsetzung „im Detail“

$>$ Herausforderungen und Grenzen

\section{Schwerpunkt 2 (Projektbeteiligte mit mehreren Teilnahmen)}

1. Welche Faktoren sind Ihrer Meinung nach ausschlaggebend für die erfolgreiche Umsetzung der Aktivitäten?

2. Wie hängen die Erfolgsfaktoren von der Zusammensetzung der ProjektpartnerInnenschaft bzw. der thematisch/inhaltlichen Ausrichtung ab?

3. Woran lässt sich der Erfolg der Aktivitäten am besten zeigen/messen?

$>$ Beobachtungen/direktes Feedback

$>$ Indikatoren/Instrumente/Evaluierungen

4. Reflexionen zum komplexen Projektdesign?

$>$ Vereinbarkeit der Zielsetzungen

$>$ Umsetzung „im Detail“

> Herausforderungen und Grenzen 